\title{
Homologia de Morse em Variedades Compactas
}

\section{Carlos Alberto Marín Arango}

\author{
Dissertação apresentada \\ ao \\ Instituto de Matemática e Estatística \\ da \\ Universidade de São Paulo \\ para \\ obtenção do grau \\ de \\ Mestre em Matemática \\ Área de Concentração: Matemática \\ Orientador : Prof. Dr. Daniel Victor Tausk \\ São Paulo, Maio de 2004
}




\section{Homologia de Morse em Variedades Compactas}

Este exemplar corresponde à redação final da dissertação de mestrado devidamente corrigida

e defendida por Carlos Alberto Marín Arango e aprovada pela comissão julgadora.

São Paulo, 7 de Maio de 2004.

Banca examinadora:

- Prof. Dr. Daniel Victor Tausk (Orientador) - IME - USP

- Prof. Dr. Paolo Piccioni - IME - USP

- Prof. Dr. Francesco Mercuri - UNICAMP 


\section{Agradecimentos}

À minha esposa Xiomara, pelo amor e a paciência que despendeu durante todo este tempo. Ao meu filho Sebastian, cuja imagem sempre decora meus pensamientos. Á minha mãe, pela luta constante para contribuir na minha formação. Voces são os deuses do meus dias, sua recordação me deu fortaleza nos momentos difícies.

Ao meu orientador Daniel, pessoa que admiro por todas as qualidades humanas e profissionais que possui, sou grato pela paciência, confiança, amizade; sobretudo pela inmensa contribução na minha formação acadêmica durante todo o período de orientação.

Em termos mais gerais, fui também bastante estimulado por um grupo de amigos, cujo entusiasmo e amizade foram uma importante ajuda. Não podendo mencionar a todos, gostaria pelo menos de destacar a Mauricio, Robson, Fernanda e os meninos da patrulha MA.

No plano da elaboração do texto, sou grato aos professores da banca examinadora Prof. Dr Paolo Piccioni (IME-USP) e Prof. Dr Francesco Mercuri (Unicamp) pelas sugestōes dispensadas à este trabalho.

Finalmente mas não por isto menos importante, à Capes, pelo apoio financiero. 


\section{Resumo}

Se $(M, g)$ é uma variedade Riemanniana compacta e $f: M \rightarrow \mathbb{R}$ é uma função de Morse cujo fluxo do campo gradiente-satisfaz a condição de Morse-Smale, nós associamos a tal fluxo um complexo de cadeia, ao qual chamamos complexo de Morse-Witten. Nós mostramos que a homologia do complexo de Morse-Witten é isomorfa a homologia singular de $M$. 


\section{Abstract}

Given a compact Riemannian manifold $(M, g)$ and a Morse function $f: M \rightarrow \mathbb{R}$ whose gradient flow satisfies the Morse-Smale condition, we construct a chain complex called the Morse-Witten complex. We prove that the homology of the Morse-Witten complex is isomorphic to the singular homology of $M$. 
Ante cualquier texto de divulgación científica se recobra vivamente el sentimiento del absurdo, pero esta vez es un sentimiento al alcance de la mano. nacido de cosas tangibles o por lo menos demostrables, casi consolador. Ya no hay que creer porque es absurdo, sino que es absurdo porque hay que creer.

J. Cortázar. 


\section{Sumário}

1 Introdução 1

2 Preliminares em Sistemas Dinâmicos 4

2.1 Fluxo de um campo vetorial . . . . . . . . . . . . . . . . 4

2.2 Singularidades Hiperbólicas de campos vetoriais . . . . . . . . . . 8

2.3 Funções de Morse e o Campo Gradiente . . . . . . . . . . . . . . . . . . 23

2.4 Aspectos Dinâmicos do Campo Gradiente . . . . . . . . . . . . . . . 27

3 O Complexo de Morse-Witten 32

3.1 Orientação e Transversalidade . . . . . . . . . . . . . . . . 32

3.2 Transversalidade das Variedades Invariantes . . . . . . . . . . . . . . 36

3.3 O complexo de Morse-Witten . . . . . . . . . . . . . . . . . 41

3.4 Teorema do Reordenamento . . . . . . . . . . . . . . . . 42

4 Tópicos em Topologia Algébrica $\quad 47$

4.1 Homologia dos Espaços Filtrados . . . . . . . . . . . . . . . . . 47

4.2 Número de Interseção . . . . . . . . . . . . . . . . . . . . . . 52

5 Isomorfismo Entre a Homologia de Morse e a Homologia Singular 62

5.1 O Teorema Fundamental . . . . . . . . . . . . . . . . . 62 


\section{Capítulo 1}

\section{Introdução}

No intuito de esclarecer a essência do resultado deste trabalho e as origens do mesmo, faremos alguns comentários iniciais. Uma boa motivação para o estudo da teoria de Morse é o carácter interdisciplinar que esta possui, (o que tornar-se-á evidente no desenvolvimento deste trabalho) pois além de ser uma técnica útil, tem em suas entranhas idéias de longo alcance que relacionam análise, topologia, geometria diferencial, topologia algébrica e mais recentemente física-matemática. A palavra recente nos leva a pensar numa divisão que deixa de um lado as técnicas clássicas da teoria e no outro os seus desenvolvimentos mais atuais. A essência da teoria clássica na formulação de Marston Morse é uma coleção de teoremas os quais descrevem numa forma simples, mas elegante a relação entre a topologia de uma variedade diferenciável $M$ e a estrutura dos pontos críticos de uma função diferenciável definida em $M$ (vide [8]). Esta teoria possui diversas aplicações importantes à teoria de geometria de subvariedades; por exemplo, estudando o caso em que $f$ é uma função altura ou uma função distância numa subvariedade $M$ do espaço Euclideano $\mathbb{R}^{n}$ (vide [15]).

A demonstração clássica das desigualdades de Morse é baseada na idéia de estudar a variação dos subníveis $f^{a}=\{x \in M: f(x) \leq a\}, a \in \mathbb{R}$ de $f$, quando a aumenta; utilizando o fluxo do campo gradiente de $f$ com respeito a uma métrica Riemanniana arbitrária, mostra-se que $f^{a}$ é homeomorfo a $f^{b}$ quando não há valores críticos no intervalo $[a, b]$, e que $f^{b}$ é homeomorfo ao espaço $f^{a}$ com uma alça colada ao longo de sua fronteira, caso exista um ponto crítico de $f \mathrm{em} f^{-1}([a, b])$. Obtém-se assim que a variedade $M$ possui o mesmo tipo de homotopia de um CW-complexo cujo número de $k$-células coincide com o número de pontos críticos com índice de Morse $k$.

Na abordagem clássica da teoria de Morse o aspecto dinâmico do campo gradiente de $f$ não é completamente explorado. Nesta dissertação, obteremos os resultados principais da teoria de Morse clássica, através do estudo da homologia de Morse de uma função de Morse f numa variedade compacta $M$, a qual satisfaz a condiçāo genérica adicional conhecida 
como condição de Morse-Smale. A homologia de Morse de uma função $f$ é definida como sendo a homologia do complexo de Morse-Witten associado a $f$; tal complexo possui como $k$-ésimo grupo de cadeias $\mathcal{C}_{k}(f)$ o grupo abeliano livre gerado pelo conjunto dos pontos críticos de índice $k$ de $f$. O operador bordo $\partial_{k}: \mathcal{C}_{k}(f) \rightarrow \mathcal{C}_{k-1}(f)$ é definido usando a dinâmica do fluxo gradiente de $f$; mais precisamente, se $p \in M$ é um ponto crítico de índice $k$ e $q \in M$ é um ponto crítico de índice $k-1$ então o coeficiente em $q$ de $\partial_{k}(p)$ é igual a uma contagem algébrica do número de linhas de fluxo de $-\nabla f$ ligando $p$ a $q$. No caso compacto, é possível mostrar que a homologia de tal complexo de cadeia é isomorfa à homologia singular de $M$. Este é precisamente o resultado central deste trabalho. Em [13] esse resultado é mostrado verificando que a homologia de Morse satisfaz os axiomas de Eilenberg-Steenrod; conclui-se então que a homologia de Morse e a homologia singular são isomorfas através de um resultado de unicidade de teorias de homologia satisfazendo tais axiomas. A demonstração apresentada aqui é original e mais direta.

Adiante apresentaremos um esboço do conteúdo deste trabalho e da técnica que usaremos em nossa demonstração; antes de fazer isso, faremos alguns comentários históricos no intuito de conhecer as origens das idéias da homologia de Morse. Embora durante a década de 60 a teoria de Morse foi usada prioritariamente para investigar a topologia das variedades (destaca-se o trabalho de Smale para mostrar a conjetura de Poncairé em dimensão maior que 4) os primeiros e principais passos em direção à definição da homologia de Morse foram tomados antes e durante tal período. $\mathrm{Na}$ década de 40, Thom reconheceu a descomposição da variedade compacta $M, M=\bigcup_{p \in \operatorname{Crit}(f)} \mathcal{W}_{u}(p,-\nabla f)$, em que Crit $(f)$ denota o conjunto dos pontos críticos de uma função de Morse $f$ definida em $M$ e $\mathcal{W}_{u}(p,-\nabla f)$ denota a variedade instável da singularidade $p$ do campo $-\nabla f$; é claro que as variedades $\mathcal{W}_{u}(p,-\nabla f)$ são células abertas com dimensão igual ao índice de Morse de $p$, mas infelizmente, tal decomposição celular não nos dá um CW-complexo.

Durante os anos 50, Smale encontra a condição para a dinâmica do campo gradiente, a qual é chamada condição de Morse-Smale e que fornece todos os ingredientes necessários para o estudo da homologia de Morse. Diz-se que uma função de Morse $f$ satisfaz a condição de Morse-Smale quando as variedades estáveis e instáveis dos pontos críticos de $f$ interceptam-se transversalmente.

Finalmente os trabalhos de Edward Witten (1982) e Andreas Floer, com o propósito de achar uma prova da conjectura de Arnold sobre órbitas períodicas de sistemas Hamiltonianos em variedades simpléticas (vide[5]), fornecem as inspirações finais para a criação da homologia de Morse.

Fica claro que estamos frente a uma teoria de grande alcance e de uma inerente e exuberante beleza. Com o passar do tempo, nossa memória vai criando uma bliblioteca que contém os livros ou as páginas que algum dia lemos em meio a uma grande emoção (ou em alguns casos a um terrível desespero), coloco o exemplar nascente de minha incursão na Teoria de Morse na estante que contém as obras mais esplêndidas nesta intima biblioteca. 
Espero pelo menos ter sido suficientemente atingido pela beleza dos conteúdos tratados aqui para poder fazer uma descrição clara dos mesmos.

O desenvolvimento de nosso trabalho seguirá o roteiro:

- No capítulo 2 apresentamos os principais conceitos e resultados da teoria dos sistemas dinâmicos, que servirão de suporte para a compreensão dos capítulos seguintes. Introduzimos as noções de campo vetorial e de fluxo associado a tal; provamos o teorema da variedade estável. Nas seções 3 e 4 definimos as noções básicas da teoria de Morse e trabalhamos os aspectos dinâmicos do campo gradiente.

- No capítulo 3 está o ingrediente principal para o desenvolvimento do nosso trabalho, a definiçao do complexo de Morse--Witten. Além do mais provamos o teorema do reordenamento que nos permite reduzir a prova do teorema central ao caso de funçôes auto-indexantes, i.e., funções tais que $f(p)$ é igual ao índice de Morse de $p$ para todo ponto crítico $p$ de $f$.

- No capítulo 4 apresentamos alguns tópicos em topologia algébrica referentes à homologia de espaços filtrados, em particular provamos que a homologia associada a uma boa filtração para um espaço topológico $X$ é naturalmente isomorfa à homologia singular de $X$. Além diso, fazemos um tratamento homológico para o conceito de número de interseção de variedades, mostrando que o mesmo coincide com o conceito diferenciável de número de interseção.

- Finalmente, no capítulo 5, provamos o resultado central. A idéia da prova será mostrar que se $f$ é uma função auto-indexante então o complexo de cadeia associado à filtração $\left(f^{k}\right)_{k \in \mathbb{N}}$ de $M$ é isomorfo ao complexo de Morse-Witten. Observamos que, com essa abordagem, a demonstração da condição $\partial_{k} \circ \partial_{k+1}=0$ que garante que o complexo de Morse-Witten é de fato um complexo de cadeia, é imediata (compare com [13]). Concluímos então que a homologia de Morse de $f$ é isomorfa a homologia singular de $M$ através dos resultados gerais de topologia algébrica estabelecidos no capítulo 4 . 


\section{Capítulo 2}

\section{Preliminares em Sistemas Dinâmicos}

Este capítulo destina-se a estabelecer a linguagem e os fatos básicos à compreensão das noções dos sistemas dinâmicos empregados no trabalho. Estabeleceremos a notação, e provaremos alguns dos principais teoremas cujos resultados serão empregados no resto deste.

\subsection{Fluxo de um campo vetorial}

Nesta seção se introduzem alguns conceitos elementares, de início enunciamos os aspectos relacionados com os campos vetoriais definidos em uma variedade diferenciável e traduzimos para este contexto os resultados da teoria das equações diferenciais; definindo a noção de curva integral, fluxo local e global. Ressaltamos também que todos os resultados, inclusive es demostrações, podem ser encontradas em [1] e [10]. Fica subentendida a seguinte notação a qual será empregada a o longo do trabalho.

Se $M$ é uma variedade diferenciável de classe $C^{k}$ e dimensão $n$, e $p \in M . T_{p} M$ denotará o espaço tangente à $M$ no ponto $p$, o qual é sabido é um espaço vetorial com dimensão $n$. Se $M, N$ são variedades diferenciáveis, $f: M \rightarrow N$ é uma aplicação de classe $C^{k}$ e $p \in M$, a aplicação linear d $f(p): T_{p} M \rightarrow T_{f(p)} N$ denotará o operador diferencial de $f$ no ponto p. $T M$ denotará o fibrado tangente de $M$, mais precisamente $T M=\bigcup_{x \in M}\{x\} \times T_{x} M$, o qual também é uma variedade diferenciável de classe $C^{k-1}$ e dimensão $2 n$.

Iniciamos aqui o estudo de alguns conceitos relacionados com as equações diferenciais; tal estudo envolve aspectos tanto locais como globais. Neste sentido o ambiente natural a ser considerado é uma variedade diferenciável.

Definição 2.1.1. Seja $M$ uma variedade diferenciável. Um campo vetorial em $M$ é uma aplicą̧ăo $\xi: M \rightarrow T M$ que a cada ponto $p \in M$ associa um vetor $\xi(p) \in T_{p} M$. 
A definição acima corresponde a uma aplicação $\xi: M \rightarrow T M$ tal que $\pi \circ \xi=I_{M}$, em que $\pi$ denota a projeção natural de $T M$ em $M$. O conjunto dos campos vetoriais de classe $C^{k}$ definidos em $M$ é denotado por $\mathfrak{X}^{k}(M)$, e por $\mathfrak{X}(M)$ é denotado o conjunto dos campos de classe $C^{\infty}$.

Se $f: M \rightarrow N$ é um difeomorfismo de classe $C^{k+1}$, e $\xi \in \mathfrak{X}^{k}(M)$, então é possível definir um elemento em $\mathfrak{X}^{k}(N)$ como:

$$
\tilde{\xi}(q)=\mathrm{d} f(p) \cdot \xi(p)
$$

em que $p=f^{-1}(q)$.

Em particular, dada uma carta $\varphi: U \subset M \rightarrow \widetilde{U} \subset \mathbb{R}^{n}$ em $M$, a aplicação $\tilde{\xi}: \widetilde{U} \rightarrow \mathbb{R}^{n}$ definida como:

$$
\tilde{\xi}(z)=\mathrm{d} \varphi\left(\varphi^{-1}(z)\right) \cdot \xi\left(\varphi^{-1}(z)\right)
$$

é um elemento de $\mathfrak{X}^{k}(\widetilde{U})$, e é chamado a representação local do campo $\xi$ na carta $\varphi$. A partir do anterior, podemos dizer que um campo vetorial $\xi$ é de classe $C^{k}$, se para cada carta $\varphi$ em $M$, a representação de $\xi$ é de classe $C^{k}$.

Se $M$ é uma variedade diferenciável e $\varphi: U \subset M \rightarrow \widetilde{U} \subset \mathbb{R}^{n}$ é uma carta em $M$, como $\varphi$ é um difeomorfismo, é possível associar uma única base $\left(\partial_{1}(p), \cdots, \partial_{n}(p)\right)$ para $o$ espaço tangente à $M$ em $p, T_{p} M$ para cada $p \in U$, basta fazer

$$
\partial_{i}(p)=\mathrm{d} \varphi(p)^{-1} \cdot e_{i} \text { para cada } i=1, \cdots, n
$$

em que $\left(e_{i}\right)_{i=1}^{n}$ denota a base canônica de $\mathbb{R}^{n}$. Além disso, a $n$-tupla $\mathrm{d} \varphi(p) \cdot v$ em $\mathbb{R}^{n}$ coincide com as componentes de $v$ na base $\left(\partial_{1}(p), \ldots, \partial_{n}(p)\right)$; em particular $\tilde{\xi}(\varphi(p)) \in \mathbb{R}^{n}$ consiste das coordenadas de $\xi(p)$ na base $\left(\partial_{1}(p), \ldots, \partial_{n}(p)\right)$ para cada $p \in U$.

Definição 2.1.2. Uma curva integral de um campo $\xi \in \mathfrak{X}^{k}(M)$ passando pelo ponto $p \in M$, é uma curva diferenciável $(I, \alpha)$, em que $I \subset \mathbb{R}$ é um intervalo aberto e $\alpha: I \rightarrow M$ é tal que $\alpha(0)=p$, e $\alpha^{\prime}(t)=\xi(\alpha(t))$ para cada $t \in I$.

Observamos que se $\xi$ é um campo vetorial em $M$, e $\tilde{\xi}$ é a representação de $\xi$ numa carta $\varphi$ em $M$, uma curva $(I, \alpha)$, é uma curva integral do campo $\xi$ quando

$$
\frac{\mathrm{d}(\varphi \circ \alpha)(t)}{\mathrm{d} t}=\tilde{\xi}(\varphi \circ \alpha(t))
$$

Definição 2.1.3. Se $\xi$ é um campo vetorial definido numa variedade $M$, um fuxo local de $\xi$ em $p \in M$ é uma aplicaçăo $F:(-a, a) \times U_{0} \rightarrow M$, em que $U_{0}$ é uma vizinhança de $p$ em $M,(-a, a) \subset \mathbb{R}$ e tal que $\forall u \in U_{0}$, a função $\alpha_{u}:(-a, a) \rightarrow M$ definida por $\alpha_{u}(t)=F(t, u)$ é uma curva integral para $\xi$ passando por $u$.

O seguinte teorema nos garante a existência e a unicidade dos fluxos locais associados a campos vetoriais. 
Teorema 2.1.4. Seja $\xi \in \mathfrak{X}^{k}(M)$, entäo para cada $x_{0} \in M$,

1. Existe uma curva diferenciável $\alpha: I \rightarrow M$ tal que:

$$
\left\{\begin{array}{l}
\alpha(0)=x_{0} \\
\alpha^{\prime}(t)=\xi(\alpha(t)) \quad \text { para cada } t \in I
\end{array}\right.
$$

2. Qualquer duas curvas integrais para $\xi$ passando por $x_{0}$ coincidem na interseção de seus domínios;

3. Existem uma vizinhança $U_{0}$ de $x_{0}$ em $M$, um número real $a \geq 0$, e uma função $F:(-a, a) \times U_{0} \rightarrow M$, de classe $C^{k}$ tal que a curva $\alpha_{u}:(-a, a) \rightarrow M$ definida como $\alpha_{u}(t)=F(t, u)$ é uma curva integral para $\xi$ passando por u em $M$.

Agora dado $p \in M$, considerando o conjunto de todas as curvas integrais em $M$ passando por $p,\left\{\left(J_{k}, \alpha_{k}\right)\right\}_{k \in \Lambda}$. Seja $\mathcal{J}_{p}=\bigcup_{k \in \Lambda} J_{k}$, então $\mathcal{J}_{p}$ é um intervalo aberto, e dado um $t \in \mathcal{J}_{p}$, é definida a curva $\sigma_{p}: \mathcal{J}_{p} \rightarrow M$ como $\sigma_{p}(t)=\alpha_{k}(t)$ para algum $k \in$ $\Lambda$, tal que $t \in J_{k}$. O teorema (2.1.4) garante que $\sigma_{p}$ é bem definida; a curva $\left(\mathcal{J}_{p}, \sigma_{p}\right)$ é chamada a curva integral maximal para $\xi$ passando por $p$.

Observação 2.1.5. Como $\mathcal{J}_{p}=\left(a_{p}, b_{p}\right)$ é o maior intervalo de definição da curva $\sigma_{p}$, se $t_{1} \in \mathcal{J}_{p}$, a curva integral maximal $\beta$ passando por $x_{1}=\sigma_{p}\left(t_{1}\right) \in U$, tem como seu maior intervalo de definição o intervalo $\mathcal{J}_{x_{1}}=\mathcal{J}_{p}-t_{1}$, e para cada $t \in \mathcal{J}_{x_{1}}, \beta(t)=\sigma_{p}\left(t+t_{1}\right)$.

Dado $\xi \in \mathfrak{X}^{k}(M)$ é possível considerar o conjunto:

$$
\mathcal{A}=\left\{(t, x) \in \mathbb{R} \times M: t \in \mathcal{J}_{x}\right\}
$$

e definir una aplicação:

$$
\begin{aligned}
F: \mathcal{A} & \longrightarrow M \\
(t, x) & \longmapsto \sigma_{x}(t)
\end{aligned}
$$

em que $\sigma_{x}$ denota a curva integral maximal passando por $x$.

Definição 2.1.6. A aplicação $F$ definida en (2.3) é chamada o fluxo global do campo $\xi$, e o conjunto $\mathcal{A}$ é chamado o dominio de definição. Se $\mathcal{A}=\mathbb{R} \times M$, então $\xi$ é dito um campo completo.

Teorema 2.1.7. Se $\xi$ é um campo vetorial de classe $C^{k}$ definido numa variedade $M$, entâo,

1. O conjunto $\mathcal{A}$ definido em (2.2) é aberto em $\mathbb{R} \times M$;

2. A aplicação $F$ definida em (2.3), é de classe $C^{k}$. 
Dados um fluxo $F$ associado a um campo vetorial $\xi$, e $t \in \mathbb{R}$, são definidos o conjunto:

$$
\mathcal{A}_{t}=\{x \in M:(t, x) \in \mathcal{A}\}
$$

e a aplicação:

$$
\begin{aligned}
F_{t}: \mathcal{A}_{t} & \longrightarrow M \\
x_{0} & \longmapsto F\left(t, x_{0}\right)
\end{aligned}
$$

O teorema (2.1.7) garante que $\mathcal{A}_{t}$ é aberto, e que a aplicação $F_{t}: \mathcal{A}_{t} \rightarrow \mathcal{A}_{-t}$ é um difeomorfismo de classe $C^{k}, \operatorname{com}\left(F_{t}\right)^{-1}=F_{-t}$.

É claro de $(2.3)$ e $(2.5)$ que $F_{t_{1}}\left(x_{0}\right)$ é só o valor da curva integral maximal passando por $x_{0}$ avaliada em $t_{1}$; se supusermos que $\sigma_{x_{0}}\left(t_{1}\right)=x_{1}$, segue da observação (2.1.5) que a curva integral maximal passando por $x_{1}, \sigma_{x_{1}}$ é tal que $\sigma_{x_{1}}(t)=\sigma_{x_{0}}\left(t+t_{1}\right)$ e $J_{x_{1}}=J_{x}-t_{1}$, assim:

$$
F_{t}\left(F_{t_{1}}\left(x_{0}\right)\right)=F\left(t, x_{1}\right)=\sigma_{x_{1}}(t)=\sigma_{x_{0}}\left(t+t_{1}\right)=F\left(t+t_{1}, x_{0}\right)=F_{t+t_{1}}\left(x_{0}\right),
$$

quer dizer que nos casos em que faça sentido, $F_{t}\left(F_{t_{1}}\left(x_{0}\right)\right)=\left(F_{t} \circ F_{t_{1}}\right)\left(x_{0}\right)=F_{t+t_{1}}\left(x_{0}\right)$.

Em vista de que o nosso principal interesse é trabalhar com campos vetoriais definidos em variedades compactas, orientamos nosso trabalho a tais superfícies.

Sejam $M$ uma variedade compacta, $p \in M, \xi \in \mathfrak{X}^{k}(M)$ e $\sigma_{p}:(a, b) \rightarrow M$ a curva integral maximal para $\xi$ passando por $p$; seja também $\left(t_{n}\right)_{n \in \mathbb{N}}$ uma seqüência em $(a, b)$, com $t_{n} \rightarrow b$, e $b<\infty$. Como $M$ é compacta, podemos supor passando a uma subsequiencia (se precisar), que $\left\{\sigma_{p}\left(t_{n}\right)\right\}$ converge para $q \in M$. Seja $F:(-\epsilon, \epsilon) \times V_{q} \rightarrow M$ um fluxo local associado a $\xi \mathrm{em} q$, tomando $n_{0}$ tal que:

$$
b-t_{n_{0}}<\frac{\epsilon}{2} \quad \text { e } \quad \sigma_{p}\left(t_{n_{0}}\right) \in V_{q}
$$

Definimos $\sigma:\left(a, t_{n_{0}}+\epsilon\right) \rightarrow M \operatorname{como} \sigma(t)=\sigma_{p}(t)$ se $t<t_{n_{0}}$ e $\sigma(t)=F\left(t-t_{n_{0}}, \sigma_{p}\left(t_{n_{0}}\right)\right)$ se $t_{n_{0}} \leq t$, então segue de (2.6) e de $\left|t-t_{0}\right|<\epsilon$, que $\sigma$ é bem definida em qualquer caso. Além do mais, no caso em que $t_{n_{0}} \leq t$, vemos que $\sigma$ é definida como uma curva integral passando por $\sigma_{p}\left(t_{n_{0}}\right)$. Portanto, a curva $\sigma$ é uma curva integral passando por $p$ o que contradiz a maximalidade de $(a, b)$. Logo $b=+\infty$; da mesma forma é provado que $a=-\infty$. Do feito acima, segue o seguinte resultado.

Teorema 2.1.8. Seja $\xi \in \mathfrak{X}^{k}(M)$, com $M$ compacta. Então

1. $\xi$ é completo:

D. Dado $t \in \mathbb{R}$, a aplicação $F_{t}$ definida em (2.5) é um difeomorfismo $C^{k}$. 


\subsection{Singularidades Hiperbólicas de campos vetoriais}

Nesta seção são tratadas as singularidades de um campo vetorial, em especial as hiperbólicas. O nosso principal interesse é definir dois conjuntos que estam intimamente relacionados com estas, provar que estes conjuntos admitem estruturas diferenciáveis que os tornam subvariedades imersas de $M$. Será subentendido sempre que faça sentido que $F$ denota o fluxo associado com algum campo vetorial explícito no contexto.

Definição 2.2.1. Seja $\xi \in \mathfrak{X}^{k}(M)$, um ponto $p \in M$ é dito uma singularidade de $\xi$, se $\xi(p)=0$.

Se $\xi \in \mathfrak{X}^{k}(M)$, a órbita de $\xi$ por $p \in M$ é definida como o conjunto:

$$
\mathcal{O}(p)=\left\{F_{t}(p): t \in \mathbb{R}\right\} .
$$

Ou seja, a órbita do campo $\xi$ pelo ponto $p$, é simplesmente a imagem da curva integral maximal para $\xi$ passando por $p$. É claro que se $\xi(p)=0$, a órbita por $p$ se reduz a $p$, basta definir a curva integral constante $\sigma=p$.

Definição 2.2.2. O w-limite de um ponto $p \in M$, é definido como o conjunto

$$
\omega(p)=\left\{q \in M: \exists\left(t_{n}\right)_{n \in \mathbb{N}} \text { com } t_{n} \rightarrow+\infty \text { e } F_{t_{n}}(p) \rightarrow q\right\}
$$

Analogamente é definido o $\alpha$-limite de $p$ como o conjunto

$$
\alpha(p)=\left\{q \in M: \exists\left(t_{n}\right)_{n \in \mathbb{N}} \operatorname{com} t_{n} \rightarrow-\infty \text { e } F_{t_{n}}(p) \rightarrow q\right\}
$$

Intuitivamente o $\omega$-limite e o $\alpha$-limite de $p$ são os conjuntos onde morre e nasce a órbita do campo por $p$.

\section{Observação 2.2.3.}

- $O$-limite de $p$, é o w-limite de p para o campo- $-\xi$;

- Se $\tilde{p}$ é um ponto da órbita por p, então $\omega(p)=\omega(\tilde{p})$, (vide (2.1.5)). Portanto é possível definir o w-limite da órbita de $p$ como sendo $\omega(p)$.

Teorema 2.2.4. Sejam $\xi \in \mathfrak{X}^{k}(M), \operatorname{com} M$ compacta e $p \in M$, então

$$
\begin{aligned}
& \text { 1. } \omega(p) \neq \emptyset ; \\
& \text { 2. } \omega(p) \text { é fechado; } \\
& \text { 3. } \omega(p) \text { é conexo. }
\end{aligned}
$$


O teorema anterior é provado em [10], e é claro que as propriedades do enunciado são válidas para o conjunto $\alpha(p)$.

Se $V$ é um espaço vetorial real de dimenção finita, e $T: V \rightarrow V$ é um endomorfismo linear, denotamos por $\mathcal{S}(T)$ o conjunto das raízes complexas do polinômio característico de $T$; isto significa que $\mathcal{S}(T)$ é igual ao conjunto dos auto-valores da complexificação de $T$, $T_{\mathbb{C}}$, o qual é a única extenção linear de $T$ à complexificação $V_{\mathbb{C}}$ de $V$. Para $\lambda \in \mathcal{S}(T) \cap \mathbb{R}$, escrevemos:

$$
V^{\lambda}(T)=\bigcup_{n \in \mathbb{N}} \operatorname{Ker}(T-\lambda I)^{n}
$$

e para $\lambda \in \mathcal{S}(T) \backslash \mathbb{R}$ escrevemos:

$$
V^{\lambda}(T)=\left(\bigcup_{n \in \mathbb{N}} \operatorname{Ker}\left(T_{\mathbb{C}}-\lambda\right)^{n} \bigoplus \bigcup_{n \in \mathbb{N}} \operatorname{Ker}\left(T_{\mathbb{C}}-\bar{\lambda}\right)^{n}\right) \cap V
$$

ou seja que $V^{\lambda}(T)=V^{\bar{\lambda}}(T)$.

Definição 2.2.5. Seja $V$ um espaço vetorial real de dimensão finita, um endomorfismo linear $T: V \rightarrow V$ é dito hiperbólico se $\mathcal{S}(T)$ é disjunto do eixo imaginário. Sáo defnidos o auto-espaço positivo e negativo de $T$ respectivamente como:

$$
V_{+}(T)=\sum_{\substack{\lambda \in \mathcal{S}(T) \\ \Re(\lambda)>0}} V^{\lambda}(T) \quad V_{-}(T)=\sum_{\substack{\lambda \in \mathcal{S}(T) \\ \Re(\lambda)<0}} V^{\lambda}(T)
$$

em que $\Re(\lambda)$ denota a parte real de $\lambda$.

Observação 2.2 .6 .

- É verdade que toda singularidade de $\xi$ pertenece ao $\omega$-limite de alguma órbita, mas não todos os elementos dos conjuntos $\omega(p)$ são singularidades de $\xi$.

- Segue da forma canônica de Jordan que se $T: V \rightarrow V$ é um endomorfismo linear hiperbólico, então pode-se escrever $V$ como a soma $V=V_{+}(T) \oplus V_{-}(T)$.

- Se $T$ é um endomorfismo linear, o qual é simétrico com respeito a algum produto interno em $V$, então $\mathcal{S}(T) \subset \mathbb{R}$, e neste caso, $V^{\lambda}(T)=\operatorname{Ker}(T-\lambda I)$ para cada $\lambda \in \mathcal{S}(T)$.

Consideremos o espaço $\mathcal{L}\left(\mathbb{R}^{n}\right)$ das aplicações lineares em $\mathbb{R}^{n}$, munido da norma

$$
\|T\|=\sup \{\|T(v)\|:\|v\| \leq 1\} .
$$

Lema 2.2.7. Se $T \in \mathcal{L}\left(\mathbb{R}^{n}\right)$, então $\|T\|=\left\|T_{\mathbb{C}}\right\|$. 
Demonstração. Seja $v \in \mathbb{R}^{n}$ tal que $\|v\| \leq 1$, então a aplicação $T_{\mathbb{C}}$ pode ser avaliada em $(v, 0)=v$ obtendo $T_{\mathbb{C}}(v)=T(v)$. Logo

$$
\left\|T_{\mathbb{C}}\right\| \geq\left\|T_{\mathbb{C}}(v)\right\|=\|T(v)\| \quad \forall v \in \mathbb{R}^{n} \text { tal que }\|v\| \leq 1,
$$

portanto $\|T\| \leq\left\|T_{\mathbb{C}}\right\|$, por outro lado, todo elemento no domínio de $T_{\mathbb{C}}$ é da forma $z=v+i w, \operatorname{com} v, w \in \mathbb{R}^{n}$, se $\|z\| \leq 1$, então $\|v\|^{2}+\|w\|^{2} \leq 1$, além do mais,

$$
\left\|T_{\mathbb{C}}(z)\right\|^{2} \leq\|T(v)\|^{2}+\|T(w)\|^{2} \leq\|T\|^{2}\left(\|v\|^{2}+\|w\|^{2}\right) \leq\|T\|^{2} .
$$

Dado $T \in \mathcal{L}\left(\mathbb{R}^{n}\right)$, consideramos $s_{m}=\sum_{k=0}^{m} \frac{1}{k !} T^{k}$, em que $T^{0}$ denota a aplicação identidade. Da completitude de $\mathcal{L}\left(\mathbb{R}^{n}\right)$, segue que $\left\{s_{m}\right\}$ é convergente; portanto é bem definida a aplicação exponencial, Exp $: \mathcal{L}\left(\mathbb{R}^{n}\right) \rightarrow \mathcal{L}\left(\mathbb{R}^{n}\right)$ como:

$$
\operatorname{Exp}(T)=e^{T}=\sum_{k=0}^{\infty} \frac{1}{k !} T^{k}
$$

Lema 2.2.8. Se $A, B \in \mathcal{L}\left(\mathbb{R}^{n}\right)$ são tais que $A B=B A$, então

$$
e^{A+B}=e^{A} e^{B} .
$$

Demonstração. Seja $s_{m}(t)=\sum_{k=0}^{m} \frac{t^{k}}{k !} A^{k}$; pela hipóteses $A B=B A$, então $A^{k} B=B A^{k} \mathrm{e}$ portanto $s_{m}(t) B=B s_{m}(t)$, de (2.8) e passando ao limite segue que:

$$
e^{t A} B=B e^{t A}
$$

Seja $x \in \mathbb{R}^{n}$, e consideremos as curvas $\alpha, \beta: \mathbb{R} \rightarrow \mathbb{R}^{n}$ definidas como

$$
\alpha(t)=e^{t(A+B)} x \text { e } \beta(t)=e^{t \cdot A} e^{i \cdot B} x
$$

é claro que:

$$
\alpha^{\prime}(t)=(A+B) \alpha(t) \text { e que } \beta^{\prime}(t)=(A+B) \beta(t)
$$

portanto $\alpha$ e $\beta$ são curvas integrais do campo linear $A+B, \operatorname{com} \alpha(0)=\beta(0)=x$, da unicidade das curvas segue que $\alpha(t)=\beta(t)$ para cada $t \in \mathbb{R}$. Em particular

$$
e^{(A+B)} x=e^{A} e^{B} x
$$

a anterior igualdade é certa para todo $x \in \mathbb{R}^{n}$, daí $e^{A+B}=e^{A} e^{B}$

Lema 2.2.9. Se $T \in \mathcal{L}\left(\mathbb{R}^{n}\right)$ e $\lambda_{0}, \lambda_{1} \in \mathbb{R}$ tais que:

$$
\lambda_{0}<\min _{\lambda \in \mathcal{S}(T)} \Re(\lambda)<\max _{\lambda \in \mathcal{S}(T)} \Re(\lambda)<\lambda_{1} .
$$

Então, existe uma constante $\mathcal{C}_{0}>0$ tal que:

$$
\left\|e^{t T}\right\| \leq \mathcal{C}_{0} e^{t \lambda_{1}}, \quad\left\|e^{-t T}\right\| \leq \mathcal{C}_{0} e^{-t \lambda_{0}}
$$

para cada $t \geq 0$. 
Demonstração. Seja $T=S+N$ a descomposição de Jordan para $T$, com $S$ semi-simple e $N$ nilpotente. Então, para cada $t \in \mathbb{R}$

$$
e^{t T}=e^{t S} \cdot e^{t N} \quad \text { e } \quad e^{t N}=I+t N+\frac{t^{2} N^{2}}{2}+\cdots+\frac{t^{n} N^{n}}{n !} .
$$

Ou seja, para cada $t>0$

$$
\left\|e^{t N}\right\| \leq 1+t\|N\|+\cdots+\frac{t^{n}\|N\|^{n}}{n !}
$$

daí, dado $\epsilon>0$ é possível achar $\mathcal{C}_{0}>0$ tal que:

$$
\left\|e^{t N}\right\| \leq \mathcal{C}_{0} e^{\epsilon t}, \text { para cada } t \geq 0
$$

Por outro lado, $\mathcal{S}(T)=\mathcal{S}(S), S_{\mathbb{C}}($ a complexificação de $S)$ é diagonalizável e:

$$
\left\|e^{t S}\right\|=\left\|e^{t S_{\mathbb{C}}}\right\| \leq c \max _{\lambda \in \mathcal{S}(T)} e^{t \Re(\lambda)}, \text { para cada } t \in \mathbb{R}
$$

onde $c$ é uma constante. Escolhemos $\epsilon>0$ tal que para cada $\lambda \in \mathcal{S}(T), \Re(\lambda)+\epsilon \leq \lambda_{1}$; segue-se de (2.9) e (2.10) que para cada $t \geq 0$ :

$$
\left\|e^{t T}\right\|=\left\|e^{t S}\right\|\left\|e^{t N}\right\| \leq \mathcal{C}_{0} e^{t \lambda_{1}}
$$

Analogamente provamos a outra desigualdade.

Lema 2.2.10. Seja $T: \mathbb{R}^{n} \rightarrow \mathbb{R}^{n}$ um operador linear hiperbólico, se denotamos por $\pi_{+}: \mathbb{R}^{n} \rightarrow \mathbb{R}_{+}^{n}(T), \pi_{-}: \mathbb{R}^{n} \rightarrow \mathbb{R}_{-}^{n}(T)$ as projeções correspondentes à decomposição $\mathbb{R}^{n}=\mathbb{R}_{+}^{n}(T) \oplus \mathbb{R}_{-}^{n}(T)$. Então, para cada $x_{0} \in \mathbb{R}_{-}^{n}(T)$, e cada aplicação contínua

$$
\mu:[0,+\infty) \longrightarrow \mathbb{R}^{n} \operatorname{com} \lim _{t \rightarrow+\infty} \mu(t)=0,
$$

existe uma única solução $x:[0,+\infty) \rightarrow \mathbb{R}^{n}$ da EDO linear não homogênea:

$$
x^{\prime}=T x+\mu
$$

$\operatorname{com} \pi_{-}(x(0))=x_{0}$ e $\lim _{t \rightarrow+\infty} x(t)=0$.

Demonstração. Denotamos por $T_{+}$e $T_{-}$os endomorfismos de $\mathbb{R}_{+}^{n}(T)$ e de $\mathbb{R}_{-}^{n}(T)$ dados pelas restrições de $T$, escolhemos $\lambda_{+}$e $\lambda_{-}$em $\mathbb{R}$ tais que:

$$
\max _{\lambda \in \mathcal{S}\left(T_{-}\right)} \Re(\lambda)<\lambda_{-}<0<\lambda_{+}<\min _{\lambda \in \mathcal{S}\left(T_{+}\right)} \Re(\lambda) \text {. }
$$

Para cada $t \geq 0$, seja

$$
x(t)=e^{t T}\left(x_{0}+\int_{0}^{t} e^{-s T} \pi_{-}(\mu(s)) \mathrm{d} s-\int_{t}^{+\infty} e^{-s T} \pi_{+}(\mu(s)) \mathrm{d} s\right)
$$


Segue do lema (2.2.9) que:

$$
\left\|e^{-s T_{+}} \pi_{+}(\mu(s))\right\|=\left\|e^{-s T_{+}} \pi_{+}(\mu(s))\right\| \leq \mathcal{C} e^{-s \lambda}\|\mu(s)\|,
$$

para todo $s \geq 0$, e alguma constante positiva $\mathcal{C}$. Mas $\lim _{t \rightarrow+\infty} \mu(t)=0$, $\mu$ é limitada, portanto a segunda integral em (2.12) converge. Uma conta simples mostra que (2.12) é solução de (2.11) com $\pi_{-}(x(0))=x_{0}$. Reescrevendo (2.12) como:

$$
x(t)^{\infty}=e^{t T} x_{0}+\int_{-\infty}^{\infty} e^{s T}\left[\mathcal{X}(s) \pi_{-}(\mu(t-s))-\mathcal{X}(-s) \pi_{+}(\mu(t-s))\right] \mathrm{d} s
$$

em que $\mathcal{X}$ denota a função característica em $[0,+\infty)$ e escrevendo $\mu(s)=0$ se $s<0$, como $x_{0} \in \mathbb{R}_{-}^{n}(T)$

$$
\left\|e^{t T} x_{0}\right\|=\left\|e^{t T_{-}} x_{0}\right\| \leq \mathcal{C} e^{t \lambda_{-}}\left\|x_{0}\right\|
$$

para cada $t \geq 0$ e alguma constante positiva $\mathcal{C}$. Logo, $\lim _{t \rightarrow+\infty} e^{t T} x_{0}=0$. Empregando o teorema de Lebesgue calculamos o limite quando $t \rightarrow+\infty$ da integral em (2.13). Como $\lim _{t \rightarrow+\infty} \mu(t)=0$, então $\lim _{t \rightarrow+\infty} e^{s T}\left[\mathcal{X}(s) \pi_{-}(\mu(t-s))-\mathcal{X}(-s) \pi_{+}(\mu(t-s))\right]=0$ para $s \in \mathbb{R}$ fixo; mas para cada $t \geq 0$ e $s \in \mathbb{R}$ temos:

$$
\left\|e^{s T}\left[\mathcal{X}(s) \pi_{-}(\mu(t-s))-\mathcal{X}(-s) \pi_{+}(\mu(t-s))\right]\right\| \leq \mathcal{C}\left(\mathcal{X}(s) e^{s \lambda_{-}}+\mathcal{X}(-s) e^{s \lambda_{+}}\right)\|\mu\|_{C_{0}}
$$

para alguma constante positva $\mathcal{C}$, em que $\|\mu\|_{C_{0}}=\sup _{s \geq 0}\|\mu(s)\|$. Obviamente

$$
\int_{-\infty}^{+\infty}\left(\mathcal{X}(s) e^{s \lambda_{-}}+\mathcal{X}(-s) e^{s \lambda_{+}}\right) \mathrm{d} s<+\infty
$$

o que completa a prova. Se $x_{1}$ e $x_{2}$ são soluções de $(2.11) \operatorname{com} \pi_{-}\left(x_{1}(0)\right)=\pi_{-}\left(x_{2}(0)\right)=x_{0}$ e $\lim _{t \rightarrow+\infty} x_{1}(t)=\lim _{t \rightarrow+\infty} x_{2}(t)=0$, então $x=x_{1}-x_{2}$ é uma solução da equação homogenea $x^{\prime}=T x \operatorname{com} x(0) \in \mathbb{R}_{+}^{n}(T)$ e $\lim _{t \rightarrow+\infty} x(t)=0$; ou seja, $x(t)=e^{i T} x(0) \epsilon$ $\mathbb{R}_{+}^{n}(T)$ para cada $t \geq 0 \mathrm{e}$

$$
\|x(0)\|=\left\|e^{-t T} x(t)\right\|=\left\|e^{-t T_{+}} x(t)\right\| \leq e^{-t \lambda_{+}}\|x(t)\| ;
$$

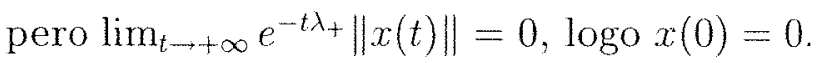

Observação 2.2.11. Sejam $p \in M$ e $L: T_{p} M \rightarrow T_{p} M$ um operador linear. Dada uma carta $\varphi: U \rightarrow \widetilde{U} \subset \mathbb{R}^{n}$ em $M$ com $p \in U$, o operador linear que representa $L$ na carta $\varphi$ é o operador linear $\tilde{L}: \mathbb{R}^{n} \rightarrow \mathbb{R}^{n}$ definido por:

$$
\tilde{L}=\mathrm{d} \varphi(p) \circ L \circ \mathrm{d} \varphi(p)^{-1} \text {. }
$$

Dados $L_{1}, L_{2}: T_{p} M \rightarrow T_{p} M$ operadores lineares e $\varphi: U \rightarrow \widetilde{U}, \psi: V \rightarrow \widetilde{V}$ cartas em $M$ com $p \in U \cap V$. Denotando por $\tilde{L}_{1}$ o operador linear em $\mathbb{R}^{n}$ que representa $L_{1}$ na carta $\varphi$ e por $\tilde{L}_{2}$ o operador linear em $\mathbb{R}^{n}$ que representa $L_{2}$ na carta $\psi$, então

$$
L_{1}=L_{2} \text { se, e somente se, } \tilde{L}_{2}=H \circ \tilde{L}_{1} \circ H^{-1}
$$

em que $H: \mathbb{R}^{n} \rightarrow \mathbb{R}^{n}$ denota a diferencial da funçăo de transição $\psi \circ \varphi^{-1}$ no ponto $\varphi(p)$. 
Sejam $\xi \in \mathfrak{X}^{k}(M), p \in M$ uma singularidade de $\xi$, dada uma carta $\varphi: U \rightarrow \widetilde{U} \subset \mathbb{R}^{n}$ em $M$ com $p \in U$, definimos o operador linear $\mathrm{d} \xi(p): T_{p} M \rightarrow T_{p} M$ como:

$$
\mathrm{d} \xi(p)=\mathrm{d} \varphi(p)^{-1} \circ \mathrm{d} \tilde{\xi}(\varphi(p)) \circ \mathrm{d} \varphi(p)
$$

em que $\tilde{\xi}$ denota a representação de $\xi$ na carta $\varphi$. Empregando a observação (2.2.11) é fácil ver que a definição em (2.14) não depende da escolha da carta.

Definição 2.2.12. Seja $\xi \in \mathfrak{X}^{k}(M)$. Uma singularidade $p \in M$ de $\xi$ é dita hiperbólica, se o endomorfismo linear em $T_{p} M, \mathrm{~d} \xi(p)$ definido em (2.14) é hiperbólico.

Definição 2.2.13. Sejam $\xi \in \mathfrak{X}^{k}(M)$ e $p \in M$ uma singularidade de $\xi$, então definimos os seguintes conjuntos:

$$
\begin{aligned}
& \mathcal{W}_{s}(p, \xi)=\left\{x \in M: x \in \mathcal{A}_{t}, \forall t \geq 0 \text { e } \lim _{t \rightarrow+\infty} F_{t}(x)=p\right\} \\
& \mathcal{W}_{u}(p, \xi)=\left\{x \in M: x \in \mathcal{A}_{t}, \forall t \leq 0 \text { e } \lim _{t \rightarrow-\infty} F_{t}(x)=p\right\}
\end{aligned}
$$

chamados variedade estável e variedade instável de p respectivamente.

É claro que $\mathcal{W}_{s}(p, \xi)=\mathcal{W}_{u}(p,-\xi)$, portanto, os resultado obtidos para o conjunto $\mathcal{W}_{s}(p, \xi)$ podem ser obtidos para o conjunto $\mathcal{W}_{u}(p, \xi)$ trocando o campo $\xi$ pelo campo $-\xi$

Vamos provar que no caso em que $p \in M$ é uma singularidade hiperbólica do campo $\xi$, existe uma estrutura diferenciável $\mathcal{A}$ com a qual $\mathcal{W}_{s}(p, \xi)$ é uma subvariedade imersa de $M$.

Se $U$ é uma vizinhança aberta de $p$ em $M$, é possivel considerar o campo $\xi$ restrito a $U$, neste caso a variedade estável de $p, \mathcal{W}_{s}\left(p,\left.\xi\right|_{U}\right)$ é denotada como $\mathcal{W}_{s}(p, U)$. Observe que $\mathcal{W}_{s}(p, U)=\left\{x \in \mathcal{W}_{s}(p, \xi): F_{t}(x) \in U, \forall t \geq 0\right\}$. De forma análoga é definido $\mathcal{W}_{u}(p, U)$.

Se $x \in \mathcal{W}_{s}(p, \xi) \cap \mathcal{A}_{t_{1}}$ para algum $t_{1} \in \mathbb{R}$, então $F_{t_{1}}(x)=\sigma_{x}\left(t_{1}\right)$ é só a imagem da curva integral maximal passando por $x$ no instante $t_{1}$, segue da observação (2.2.3) que $\sigma_{x}\left(t_{1}\right)$ é de novo um elemento de $\mathcal{W}_{s}(p, \xi)$. Portanto o conjunto $\mathcal{W}_{s}(p, \xi)$ é invariante pelo fluxo de $\xi$.

\section{Observação 2.2.14.}

- $O$ difeomorfismo $F_{t}$ restrito a $\mathcal{W}_{s}(p, \xi) \cap \mathcal{A}_{t}$ é um homeomorfismo, sobre $\mathcal{W}_{s}(p, \xi) \cap$ $\mathcal{A}_{-t}$, assim, se $Z$ é um aberto em $\mathcal{W}_{s}(p, \xi)$, então $F_{t}^{-1}\left(Z \cap \mathcal{A}_{-t}\right)=F_{-t}\left(Z \cap \mathcal{A}_{-t}\right)$ e $F_{t}\left(Z \cap \mathcal{A}_{t}\right)$ são abertos em $\mathcal{W}_{s}(p, \xi)$.

- O conjunto $\mathcal{W}_{s}(p, \xi)$ é conexo por arcos, (com respeito a topologia induzida de $M$ ). Pois se $x \in \mathcal{W}_{s}(p, \xi)$, então

$$
\alpha(t)=\left\{\begin{array}{lc}
F\left(\frac{t}{1-t^{2}}, x\right), & t \in[0,1) \\
p, & t=1
\end{array}\right.
$$


define uma curva contínua $\alpha:[0,1] \rightarrow \mathcal{W}_{s}(p, \xi)$ ligando $x$ e $p$.

Dados abertos $U$ e $V$ em $M \operatorname{com} p \in U \cap V$, segue da definição anterior que

$$
\mathcal{W}_{s}(p, U) \cap \mathcal{W}_{s}(p, V)=\mathcal{W}_{s}(p, U \cap V)
$$

É também fácil provar que para qualquer vizinhança $U \subset M$ de $p$, e para todo $t \in \mathbb{R}$ :

$$
\begin{gathered}
F_{t}\left(\mathcal{W}_{s}(p, U) \cap \mathcal{A}_{t}\right)=\mathcal{W}_{s}\left(p, F_{t}\left(U \cap \mathcal{A}_{t}\right)\right) \\
F_{t}^{-1}\left(\mathcal{W}_{s}(p, U)\right)=\mathcal{W}_{s}\left(p, F_{t}^{-1}(U)\right) \\
\mathcal{W}_{s}(p, \xi)=\bigcup_{t \geq 0} F_{t}^{-1}\left(\mathcal{W}_{s}(p, U)\right)=\bigcup_{t \geq 0} \mathcal{W}_{s}\left(p, F_{t}^{-1}(U)\right) .
\end{gathered}
$$

Com efeito, sejam $t_{0} \in \mathbb{R}$ fixo, e $x \in \mathcal{W}_{s}(p, U) \cap \mathcal{A}_{t_{0}}$, da definição acima

$$
x \in \mathcal{W}_{s}(p, \xi), F_{t}(x) \in U, \forall t, t \geq 0 \text { e } x \in \mathcal{A}_{t_{0}} .
$$

Vejamos que $F_{t_{0}}(x) \in \mathcal{W}_{s}\left(p, F_{t_{0}}\left(U \cap \mathcal{A}_{t_{0}}\right)\right)$, ou seja, que

$$
F_{t_{0}}(x) \in \mathcal{W}_{s}(p, \xi) \text { e } F_{t}\left(F_{t_{0}}(x)\right) \in F_{t_{0}}\left(U \cap \mathcal{A}_{t_{0}}\right) \forall t \geq 0
$$

Como $x \in \mathcal{A}_{t_{0}}$, faz sentido falar de $F_{t_{0}}(x)$, além do mais, $F_{t_{0}}(x)$ é um elemento da órbita pelo ponto $x$; pelo comentário após da definição $(2.2 .13), F_{t_{0}}(x) \in \mathcal{W}_{s}(p, \xi)$. Se $t_{1} \geq 0$, como $F_{t_{0}}(x) \in \mathcal{W}_{s}(p, \xi)$, temos que $F_{t_{0}}(x) \in \mathcal{A}_{t}$, para cada $t \geq 0$, em particular para $t_{1}$, daí

$$
F_{t_{1}}\left(F_{t_{0}}(x)\right)=F_{t_{0}}\left(F_{t_{1}}(x)\right) \text { com } F_{t_{1}}(x) \in U \text {. }
$$

Além disso, $F_{t_{1}}(x) \in \mathcal{A}_{t_{0}} ;$ pois $t_{0} \in J_{x} \subset J_{F_{t_{1}}(x)}$, assim $F_{t_{0}}(x) \in \mathcal{W}_{s}(p, \xi)$, e para cada $t \geq 0, F_{t}\left(F_{t_{0}}(x)\right)=F_{t_{0}}\left(F_{t}(x)\right) \operatorname{com} F_{t}(x) \in U \cap \mathcal{A}_{t_{0}}$.

Reciprocamente, se $x \in \mathcal{W}_{s}\left(p, F_{t_{0}}\left(U \cap \mathcal{A}_{t_{0}}\right)\right)$, então

$$
x \in \mathcal{W}_{s}(p, \xi) \text {, e para cada } t \geq 0, F_{t}(x) \in F_{t_{0}}\left(U \cap \mathcal{A}_{t_{0}}\right)
$$

Vejamos que $x=F_{t_{0}}(y)$, com $y \in \mathcal{W}_{s}(p, \xi), F_{t}(y) \in \mathcal{A}_{t}$ para cada $t \geq 0$ e $F_{t_{0}}$ definida em $y$. Se $t \geq 0$, pela hipóteses $F_{t}(x) \in F_{t_{0}}\left(U \cap \mathcal{A}_{t_{0}}\right)$, ou seja $F_{t}(x)=F_{t_{0}}(z)$ para algum $z \in U \cap \mathcal{A}_{t_{0}}$, daí $x=F_{-t}\left(F_{t_{0}}(z)\right)$. Para obter a conclusão basta fazer $y=F_{-t}(z)$.

O raciocínio acima prova (2.16), da mesma forma é provado (2.17), agora para (2.18) temos

$$
\mathcal{W}_{s}(p, U) \subset \mathcal{W}_{s}(p, \xi)
$$

portanto da igualdade (2.17) segue

$$
F_{t}^{-1}\left(\mathcal{W}_{s}(p, U)\right)=\mathcal{W}_{s}\left(p, F_{t}^{-1}(U)\right) \subset \mathcal{W}_{s}(p, \xi)
$$


ou seja, que $\bigcup_{t \geq 0} F_{t}^{-1}\left(\mathcal{W}_{s}(p, U)\right) \subset \mathcal{W}_{s}(p, \xi)$. Se $x \in \mathcal{W}_{s}(p, \xi)$, pela definição (2.2.13), $\lim _{t \rightarrow \infty} F_{t}(x)=p$; daí, existe um $t_{0} \geq 0$, tal que $F_{t}(x) \in U$, para cada $t \geq t_{0}$ logo, é claro que $F_{t_{0}}(x) \in \mathcal{W}_{s}(p, U)$. Pois $F_{t_{0}}(x) \in \mathcal{W}_{s}(p, \xi)$ e $F_{t}\left(F_{t_{0}}(x)\right)=F_{t+t_{0}}(x) \in U$, para $t \geq 0$, ou seja que

$$
x \in F_{-t_{0}}\left(\mathcal{W}_{s}(p, U)\right)=F_{t_{0}}^{-1}\left(\mathcal{W}_{s}(p, U)\right) \subset \bigcup_{t \geq 0} F_{t}^{-1}\left(\mathcal{W}_{s}(p, U)\right)
$$

Observação 2.2.15. A uniâo em (2.18) é monótona, ou seja, se $0 \leq t \leq s$, então,

$$
F_{t}^{-1}\left(\mathcal{W}_{s}(p, U)\right) \subset F_{s}^{-1}\left(\mathcal{W}_{s}(p, U)\right)
$$

Entendendo por uma subvariedade de classe $C^{k}$ de uma variedade diferenciável $M$, um subconjunto $N$ de $M$ dotado de uma estrutura diferenciável de classe $C^{k}$ induzida de $M$, tal que a aplicação inclusão é um mergulho, tal subvariedade também pode-se chamar subvariedade mergulhada. Por outro lado, uma subvariedade imersa de $M$ de classe $C^{k}$ é um subconjunto $N$ de $M$, junto com uma estrutura diferenciável $\mathcal{A}$ que o torna uma variedade diferenciável de classe $C^{k}$, e tal que a aplicação inclusão de $(N, \mathcal{A})$ em $M$ é uma imersão de classe $C^{k}$. Logo toda subvariedade de $M$ é uma subvariedades imersa de $M$. No caso de uma subvariedade imersa, a topologia induzida em $N$ pela estrutura diferenciável que torna $N$ uma variedade é mais fina que a topologia subespaço induzida de $M$ em $N$.

Denotamos por $E^{0}$ o espaço de Banach das aplicações contínuas

$$
\gamma:[0,+\infty) \longrightarrow \mathbb{R}^{n} \text { tais que } \lim _{t \rightarrow+\infty} \gamma(t)=0
$$

dotado da norma $\|\gamma\|_{C_{0}}=\sup _{t \geq 0}\|\gamma(t)\|$, e por $E^{1}$ o espaço de Banach das aplicações de classe $C^{1}$

$$
\gamma:[0,+\infty) \longrightarrow \mathbb{R}^{n} \text { tais que } \lim _{t \rightarrow+\infty} \gamma(t)=\lim _{t \rightarrow+\infty} \gamma^{\prime}(t)=0
$$

dotado da norma $\|\gamma\|_{C_{1}}=\|\gamma\|_{C_{0}}+\left\|\gamma^{\prime}\right\|_{C_{0}}$.

Lema 2.2.16. Se p é uma singularidade hiperbólica de um campo vetorial $\xi$ de classe $C^{k},(1 \leq k \leq \infty)$ definido numa variedade diferenciável $M$, então existe uma vizinhança $U \subset M$ de $p$ tal que:

1. $\mathcal{W}_{s}(p, U)$ é uma subvariedade de $M$, cujo espaço tangente em $p$ é o auto-espaço negativo do operador $\mathrm{d} \xi(p)$.

2. Se $V \subset M$ é uma vizinhança de $p, \operatorname{com} V \subset U$, então $\mathcal{W}_{s}(p, V)$ é aberto em $\mathcal{W}_{s}(p, U)$, e portanto uma subvariedade de $M$. 
Demonstração. Sem perda de generalidade, é possível assumir que $M$ é uma vizinhança da origem em $\mathbb{R}^{n}$, e $p=0$. $\mathbb{R}_{+}^{n}$ e $\mathbb{R}_{-}^{n}$ denotam respectivamente o auto-espaço positivo e o autoespaço negativo de $\mathrm{d} \xi(0)$, e $\pi_{+}, \pi_{-}$as respectivas projeções com respeito à decomposição $\mathbb{R}^{n}=\mathbb{R}_{+}^{n} \oplus \mathbb{R}_{-}^{n}$. Se $U \subset \mathbb{R}^{n}$ é uma vizinhança aberta da origem, escrevemos:

$$
E^{k}(U)=\left\{\gamma \in E^{k}: \operatorname{Im}(\gamma) \subset U\right\}
$$

é claro que $E^{k}(U)$ é um subconjunto aberto de $E^{k}$ para $k=0,1$.

Consideremos a aplicação

$$
\phi: \mathbb{R}_{-}^{n} \times E^{1}(M) \longrightarrow \mathbb{R}_{-}^{n} \times E^{0}
$$

definida como $\phi\left(x_{0}, \gamma\right)=\left(\pi_{-}(\gamma(0))-x_{0}, \gamma^{\prime}-\xi \circ \gamma\right)$.

Usando técnicas padrão (vide [11]), mostra-se que a aplicação $\phi$ é de classe $C^{k}$ e sua derivada parcial $\frac{\partial \phi}{\partial \gamma}$ na origem é dada por:

$$
\frac{\partial \phi}{\partial \gamma}(0,0) v(t)=\left(\pi-(v(0)), v^{\prime}(t)-\mathrm{d} \xi(\gamma(t)) v(t)\right)
$$

para cada $v \in E^{1}$. Do lema (2.2.10), segue que $\frac{\partial \phi}{\partial \gamma}(0,0)$ é um isomorfismo, além do mais, como $\phi(0,0)=(0,0)$, segue do teorema da função implícita que existem vizinhanças abertas $B\left(0, r_{1} ; \mathbb{R}_{-}^{n}\right), B\left(0, r_{2} ; E^{1}(M)\right)$ da origem em $\mathbb{R}_{-}^{n}$ e em $E^{1}(M)$ respectivamente, e uma aplicação $\sigma: B\left(0, r_{1} ; \mathbb{R}_{-}^{n}\right) \rightarrow B\left(0, r_{2} ; E^{1}(M)\right)$ de classe $C^{k}$, tal que:

$$
\left(B\left(0, r_{1} ; \mathbb{R}^{n}\right) \times B\left(0, r_{2} ; E^{1}(M)\right)\right) \cap \phi^{-1}(0,0)=\operatorname{Gr}(\sigma) .
$$

Em que $\operatorname{Gr}(\sigma)$ denota o gráfico de $\sigma$. Dado qualquer $h \in \mathbb{R}_{-}^{n}$, seja $v=\mathrm{d} \sigma(0) h$; temos:

$$
v=-\left[\frac{\partial \phi}{\partial \gamma}(0,0)^{-1} \circ \frac{\partial \phi}{\partial x_{0}}(0,0)\right] h
$$

$\mathrm{e}$

$$
\frac{\partial \phi}{\partial x_{0}}(0,0) h=(-h, 0)
$$

daí:

$$
v=\frac{\partial \phi}{\partial \gamma}(0,0)^{-1}(h, 0)
$$

Observando $(2.20)$ e (2.22), pode-se concluir que $\left(\pi_{-}(v(0)), v^{\prime}(t)-\mathrm{d} \xi(0) v(t)\right)=(h, 0)$, em que $v:[0,+\infty) \rightarrow \mathbb{R}^{n}$, é uma solução de $v^{\prime}(t)=\mathrm{d} \xi(0) v(t), \operatorname{com} \pi_{-}(v(0))=h$ e $\lim _{t \rightarrow \infty} v(t)=0$, assim

$$
v(t)=e^{t \mathrm{~d} \xi(0)} h
$$

Agora escolhemos uma vizinhança $U$ da origem tal que

$$
\sup _{x \in U}\|x\|<\frac{r_{2}}{2}, \quad \sup _{x \in U}\|\xi(x)\|<\frac{r_{2}}{2}, \quad \sup _{x \in U}\left\|\pi_{-}(x)\right\|<r_{1}
$$


se $\gamma:[0,+\infty) \rightarrow \mathbb{R}^{n}$ é curva integral para $\xi, \operatorname{com} \operatorname{Im}(\gamma) \subset U$, e tal que $\lim _{t \rightarrow \infty} \gamma(t)=0$, então pela escolha de $U$ temos:

$$
\|\xi(\gamma(0))\|<\frac{r_{2}}{2}, \quad\|\gamma(0)\|<\frac{r_{2}}{2}, \text { e }\left\|\pi_{-}(\gamma(0))\right\|<r_{1}
$$

Ou seja, que $\pi_{-}(\gamma(0)) \in B\left(0, r_{1} ; \mathbb{R}_{-}^{n}\right)$ além disso,

$$
\|\gamma\|_{C_{1}}=\left\|\gamma^{\prime}\right\|_{C_{0}}+\|\gamma\|_{C_{0}} \leq \frac{r_{2}}{2}+\frac{r_{2}}{2}
$$

Logo $\gamma \in B\left(0, r_{2} ; E^{1}(M)\right)$. Então

$$
\mathcal{W}_{s}(0, U)=\left\{\gamma(0): \gamma \in E^{1}(U) \text { e }\left(\pi_{-}(\gamma(0)), \gamma\right) \in \operatorname{Gr}(\sigma)\right\}
$$

seja $\eta: B\left(0, r_{1} ; \mathbb{R}_{-}^{n}\right) \rightarrow \mathbb{R}_{+}^{n}$ definida como:

$$
\eta\left(x_{0}\right)=\pi_{+}(\gamma(0))
$$

em que $\gamma=\sigma\left(x_{0}\right)$ segue-se de (2.21) e (2.23) que $\mathrm{d} \eta(0)=0$, além do mais, por $(2.24)$ temos que

$$
\mathcal{W}_{s}(0, U)=\operatorname{Gr}\left(\left.\eta\right|_{\sigma^{-1}\left(E^{1}(U)\right)}\right),
$$

em que $\sigma^{-1}\left(E^{1}(U)\right)$ é um subconjunto aberto de $B\left(0, r_{1} ; \mathbb{R}_{-\ldots}^{n}\right)$. Isto prova que $\mathcal{W}_{s}(0, U)$ é uma subvariedade de $\mathbb{R}^{n}$, cujo espaço tangente em 0 é $\operatorname{Gr}(\mathrm{d} \eta(0))=\mathbb{R}_{-}^{n}$. Para a segunda afirmação, se $V \subset U$ aberto com $0 \in V$, basta observar que $\mathcal{W}_{s}(0, V)=\operatorname{Gr}\left(\left.\eta\right|_{\sigma^{-1}\left(E^{1}(V)\right)}\right)$. Assim $\mathcal{W}_{s}(0, V)$ é um subconjunto aberto de $\mathcal{W}_{s}(0, U)$, pois $\sigma^{-1}\left(E^{1}(V)\right)$ é um aberto em $B\left(0, r_{1} ; \mathbb{R}_{-}^{n}\right)$.

Sejam $U$ como no lema (2.2.16), $t \in \mathbb{R}$ e $Z$ uma vizinhança aberta de $p$ em $M$ contida em $F_{t}^{-1}(U)$, então $\mathcal{W}_{s}(p, Z)$ é aberto em $\mathcal{W}_{s}\left(p, F_{t}^{-1}(U)\right)$, logo por $(2.17), \mathcal{W}_{s}(p, Z)$ é aberto em $F_{t}^{-1}\left(\mathcal{W}_{s}(p, U)\right)$

Agora será provado que $\mathcal{W}_{s}(p, \xi)$ é uma subvariedade imersa em $M$. Para isto precisamos de mais um lema.

Lema 2.2.17. Seja $M$ uma variedade diferenciável de classe $C^{k}$, e seja $\left(N_{i}\right)_{i \in I}$ uma familia de subvariedades de $M$. Assuma que $\forall i, j \in I, N_{i} \cap N_{j}$ é uma aberto em $N_{i} e$ em $N_{j}$. Assuma também que existe um subconjunto enumerável $J \subset I$ tal que $\bigcup_{i \in J} N_{i}=$ $\bigcup_{i \in I} N_{i}$. Então existe uma única estrutura diferenciável em $N=\bigcup_{i \in I} N_{i}$, tal que $N$ é uma subvariedade imersa de $M$ e tal que para cada $i \in I, N$ é aberto em $N$ com respeito à topologia de $N$ como variedade. Além do mais, para cada $i \in I$, a estrutura de variedade diferenciável de $N_{i}$ como aberto de $N$ coincide com a estrutura de $N_{i}$ como subvariedade de $M$. 
Demonstração. Seja $N_{i}$ munido da estrutura diferenciável $\mathcal{A}_{i}=\left\{\psi_{i \lambda}: V_{i \lambda} \rightarrow \widetilde{V}_{i \lambda}\right\}_{\lambda \in \Lambda}$ de classe $C^{k}$ com a qual é uma subvariedade de $M$, definimos

$$
\mathcal{A}_{0}=\left\{\psi_{i \lambda}: V_{i \lambda} \rightarrow \widetilde{V}_{i \lambda}\right\}_{i \in I, \lambda \in \Lambda}
$$

é claro que para cada $i \in I,\left\{V_{i \lambda}\right\}_{\lambda \in \Lambda}$ cobre a $N_{i}$, portanto $\left\{V_{i \lambda}\right\}_{\lambda \in \Lambda, i \in I}$ cobre a união $N=\bigcup_{i \in I} N_{i}$. Dados $i, j \in I$, como $N_{i} \cap N_{j}$ é um aberto em $N_{i}$ e em $N_{j}$, segue que $\forall i, j \in I, \alpha, \lambda \in \Lambda, \quad V_{i \lambda} \cap V_{j \alpha}$ é uma aberto em $V_{i \lambda}$ e em $V_{j \alpha}$, e como $\psi_{i \lambda}, \psi_{j \alpha}$ são difeomorfismos $C^{k}, \psi_{i \lambda}\left(V_{i \lambda} \cap V_{j \alpha}\right)$ e $\psi_{j \alpha}\left(V_{i \lambda} \cap V_{j \alpha}\right)$ são abertos, e $\psi_{j \alpha} \circ \psi_{i \lambda}^{-1}$, $\psi_{i \lambda} \circ \psi_{j \alpha}^{-1}$ são difeomorfismos $C^{k}$. Pelo anterior, o conjunto $\mathcal{A}_{0}$ definido acima é um atlas em $N$. Se $\mathcal{A}$ é o atlas maximal de classe $C^{k}$ que contém $\mathcal{A}_{0}$, afirmamos que cada $V_{i \lambda}$ é aberto em $N$ na topologia definida por $\mathcal{A}$, e cada $\psi_{i \lambda}$ é um difeomorfismo de classe $C^{k}$ com respeito à estrutura diferenciável definida por $\mathcal{A}$. É claro que a topologia em $N$ induzida por $\mathcal{A}$ em $N$ é Hausdorff, e como $\mathcal{A}$ contém o atlas $\mathcal{A}_{J}=\left\{\psi_{i \lambda}\right\}_{i \in J, \lambda \in \Lambda_{i}}$ com $J$ enumerável, segue que a topologia induzida por $\mathcal{A}$ em $N$ satisfaz o segundo axioma de enumerabilidade. $N i=\bigcup_{\lambda \in \Lambda_{i}} V_{i \lambda}$ mostra que $N_{i}$ é aberto em $N$ com respeito à topologia de $N$ como variedade; vejamos que $N$ junto da topologia induzida por $\mathcal{A}$ é uma subvariedade imersa de $M$, ou seja, dado $x \in N$ vejamos que d $i(x)$ é injetora, isto segue facilmente dos seguintes diagramas comutativos:
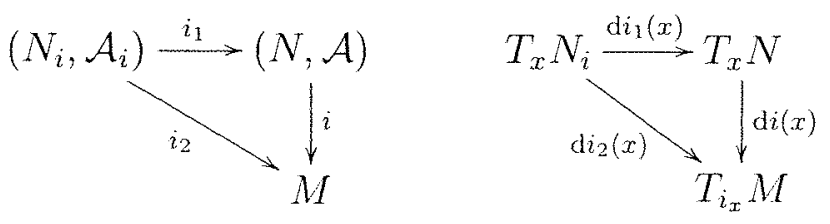

e como $N_{i}$ é aberto em $N, T_{x} N_{i}=T_{x} N$, as aplicações $i_{1}, i_{2}$ são mergulhos e d $i_{1}(x)$ é um isomorfismo, também fica claro pela definição de $\mathcal{A}$ que a estrutura diferenciável que torna $N_{i}$ uma subvariedade de $M$, coincide com a estrutura diferenciável de $N_{i}$ como aberto de $N$.

Proposição 2.2.18. Existe uma única estrutura diferenciável no conjunto $\mathcal{W}_{s}(p, \xi)$, tal que $\mathcal{W}_{s}(p, \xi)$ é uma subvariedade imersa de $M$, e tal que para cada vizinhança aberta $V \subset M$ de $p, \mathcal{W}_{s}(p, V)$ é aberto em $\mathcal{W}_{s}(p, \xi)$ com respeito à topologia de $\mathcal{W}_{s}(p, \xi)$ como variedade diferenciável. Além disso, as seguintes afirmaçôes são verdadeiras:

1. O espaço tangente de $\mathcal{W}_{s}(p, \xi)$ em $p$ é igual ao auto-espaço negativo de $\mathrm{d} \xi(p)$;

2. A restrição de $\xi$ a $\mathcal{W}_{s}(p, \xi)$ é um campo $C^{k}$;

3. Para $x \in \mathcal{W}_{s}(p, \xi)$, a curva integral maximal de $\xi$ passando por $x$ é igual à curva integral maximal de $\xi \mid \mathcal{W}_{s}(p, \xi)$ passando por $x$;

4. O ponto p é uma singularidade hiperbólica de $\left.\xi\right|_{\mathcal{W}_{s}(p, \xi)}$ cuja variedade estável é igual a $\mathcal{W}_{s}(p, \xi)$; 
Demonstração. Tomando $U$ como no lema (2.2.16). Então $\mathcal{W}_{s}(p, U)$ é subvariedade de $M$, e para cada $t \geq 0$, como $F_{t}$ é um difeomorfismo entre abertos de $M$, segue que $F_{t}^{-1}\left(\mathcal{W}_{s}(p, U)\right)$ é uma subvariedade de $M$. De $(2.18) \mathcal{W}_{s}(p, \xi)$ é uma união monótona de subvariedades de $M$. A estratégia é empregar o resultado do lema anterior, para construir uma estrutura de variedade diferenciável em $\mathcal{W}_{s}(p, \xi)$. Para iniciar observe que a união em (2.18) pode ser tomada sobre $t \in \mathbb{N}$, ou seja, ela pode ser substituída por uma união enumerável. Agora para $0 \leq t \leq s$, o conjunto $F_{t}^{-1}\left(\mathcal{W}_{s}(p, U)\right)$ é aberto em $F_{s}^{-1}\left(\mathcal{W}_{s}(p, U)\right)$. De (2.15) e (2.17) segue:

$$
\begin{aligned}
F_{t}^{-1}\left(\mathcal{W}_{s}(p, U)\right) & =F_{t}^{-1}\left(\mathcal{W}_{s}(p, U)\right) \cap F_{s}^{-1}\left(\mathcal{W}_{s}(p, U)\right) \\
& =\mathcal{W}_{s}\left(p, F_{t}^{-1}(U)\right) \cap \mathcal{W}_{s}\left(p, F_{s}^{-1}(U)\right) \\
& =\mathcal{W}_{s}\left(p, F_{t}^{-1}(U) \cap F_{s}^{-1}(U)\right)
\end{aligned}
$$

Como $F_{t}^{-1}(U) \cap F_{s}^{-1}(U)$ é uma vizinhança aberta de $p$ em $M$, contida em $F_{s}^{-1}(U)$, do comentário após de (2.2.16) segue que $\mathcal{W}_{s}\left(p, F_{t}^{-1}(U) \cap F_{s}^{-1}(U)\right)=F_{t}^{-1}\left(\mathcal{W}_{s}(p, U)\right)$ é aberto em $F_{s}^{-1}\left(\mathcal{W}_{s}(p, U)\right)$. Até aqui foi provado que:

- Existe uma estrutura de variedade diferenciável em $\mathcal{W}_{s}(p, \xi)$ com a qual é uma subvariedade imersa de $M$, e tal que para cada $t \geq 0, \mathcal{W}_{s}\left(p, F_{t}^{-1}(U)\right)$ é aberto em $\mathcal{W}_{s}(p, \xi)$.

Seja $V \subset M$ uma vizinhança aberta de $p$, de (2.15) e (2.17) segue que

$$
\mathcal{W}_{s}(p, V)=\bigcup_{t \geq 0} \mathcal{W}_{s}(p, V) \cap \mathcal{W}_{s}\left(p, F_{t}^{-1}(U)\right)=\bigcup_{t \geq 0} \mathcal{W}_{s}\left(p, V \cap F_{t}^{-1}(U)\right)
$$

Como $V \cap F_{t}^{-1}(U)$ é uma vizinhança aberta de $p$ contida em $F_{t}^{-1}(U)$, pelo comentário após de $(2.2 .16), \mathcal{W}_{s}\left(p, V \cap F_{t}^{-1}(U)\right)$ é aberto em $\mathcal{W}_{s}\left(p, F_{t}^{-1}(U)\right)$, mas $\mathcal{W}_{s}\left(p, F_{t}^{-1}(U)\right)$ é aberto em $\mathcal{W}_{s}(p, \xi)$ com respeito à topologia de subvariedade; e em $\mathcal{W}_{s}\left(p, F_{t}^{-1}(U)\right)$ as topologias induzidas de $M$ e de $\mathcal{W}_{s}(p, \xi)$ coincidem. Portanto $\mathcal{W}_{s}\left(p, V \cap F_{t}^{-1}(U)\right)$ é aberto em $\mathcal{W}_{s}(p, \xi)$ com respeito à topologia de subvariedade, e assim de $(2.25), \mathcal{W}_{s}(p, V)$ é também aberto em $\mathcal{W}_{s}(p, \xi)$ com respeito à mesma topologia, isto prova o enunciado inicial. Para o item (1) temos que $\mathcal{W}_{s}(p, U)$ é uma subvariedade aberta de $\mathcal{W}_{s}(p, \xi)$, portanto (1) segue de (2.2.16). Agora para (2) e (3); seja $x \in \mathcal{W}_{s}(p, \xi)$ fixo e denote por $\sigma_{x}: \mathbb{R} \rightarrow M$ a curva integral maximal para $\xi$ passando por $x$. É obvio que $\sigma_{x}(I) \subset \mathcal{W}_{s}(p, \xi)$, como $\lim _{t \rightarrow \infty} \sigma_{x}(t)=p$, segue que $\sigma_{x}(t) \in U$ para $t$ suficientemente grande, ou seja, dado um intervalo limitado $I \subset \mathbb{R}$, existe um $t \geq 0$ tal que $\sigma_{x}(I) \subset F_{t}^{-1}\left(\mathcal{W}_{s}(p, U)\right)$; mas $F_{t}^{-1}\left(\mathcal{W}_{s}(p, U)\right)$ é uma subvariedade de $M$ e uma subvariedade aberta de $\mathcal{W}_{s}(p, \xi)$, portanto $\left.\sigma_{x}\right|_{I}: I \rightarrow \mathcal{W}_{s}(p, \xi)$ é uma curva diferenciável, como $I$ é limitado e arbitrário, então $\sigma_{x}: \mathbb{R} \rightarrow \mathcal{W}_{s}(p, \xi)$ é diferenciável, e $\sigma_{x}^{\prime}(0)=\xi(x) \in T_{x}\left(\mathcal{W}_{s}(p, \xi)\right)$. Para provar o enunciado (4), p obviamente é uma singularidade de $\left.\xi\right|_{\mathcal{W}_{s}(p, \xi)}$ e $\mathrm{d}\left(\left.\xi\right|_{\mathcal{W}_{s}(p, \xi)}\right)(p)$ é igual à restriçâo de $\mathrm{d} \xi(p)$ a $T_{p}\left(\mathcal{W}_{s}(p, \xi)\right)$, o qual é igual ao auto-espaço negativo de $\mathrm{d} \xi(p)$. Ou seja que $p$ é uma 
singularidade hiperbólica de $\xi \mathcal{W}_{\mathcal{N}}(p, \xi)$, agora para provar que $\mathcal{W}_{s}(p, \xi)$ é a variedade estável de $p$ respeito a $\left.\xi\right|_{\mathcal{W}_{s}(p, \xi)}$, vejamos que para cada $x \in \mathcal{W}_{s}(p, \xi), \quad \lim _{t \rightarrow \infty} F_{t}(x)=p$, com respeito à topologia de $\mathcal{W}_{s}(p, \xi)$ como subvariedade, mas é sabido que $\lim _{t \rightarrow \infty} F_{t}(x)=p$ com respeito à topologia de $M, F_{t}(x) \in \mathcal{W}_{s}(p, U)$ para $t$ suficientemente grande, e a topologia de $\mathcal{W}_{s}(p, U)$ como subvariedade aberta de $\mathcal{W}_{s}(p, \xi)$ coincide com a induzida como subvariedade de $M$, portanto $\lim _{t \rightarrow \infty} F_{t}(x)=p$ com respeito à topologia de subvariedade de $\mathcal{W}_{s}(p, \xi)$

Agora a seguinte proposição fornece condições para que a variedade estável $\mathcal{W}_{s}(p, \xi)$ seja uma subvariedade mergulhada de $M$.

Proposição 2.2.19. As seguintes afirmações são equivalentes:

1. $\mathcal{W}_{s}(p, \xi)$ é uma subvariedade mergulhada de $M$;

2. Para cada vizinhança aberta $V \subset M$ de $p, \mathcal{W}_{s}(p, V)$ é aberto em $\mathcal{W}_{s}(p, \xi)$ com respeito à topologia induzida;

3. Toda vizinhança aberta $V \subset M$ de $p$, contém uma vizinhança aberta $Z \subset M$ de $p$ tal que $\mathcal{W}_{s}(p, Z)$ é aberto em $\mathcal{W}_{s}(p, \xi)$ com respeito à topologia induzida;

4. Toda vizinhança aberta $V \subset M$ de $p$, contem uma vizinhança aberta $Z \subset M$ de $p$ tal que $\mathcal{W}_{s}(p, Z)=\mathcal{W}_{s}(p, \xi) \cap Z$;

5. Existe uma vizinhança aberta $V \subset M$ de $p$ tal que $\mathcal{W}_{s}(p, \xi) \cap V$ é uma subvariedade de $M$.

Demonstração. $(1) \Rightarrow(2)$. Como $\mathcal{W}_{s}(p, \xi)$ é uma subvariedade de $M$, a estrutura diferenciável que torna tal conjunto subvariedade coincide com a estrutura da proposição anterior, isto prova (2).

$(2) \Rightarrow(3)$. É inmediato.

$(3) \Rightarrow(4)$. Seja $V \subset M$ uma vizinhança aberta de $p$, por (3), existe uma vizinhança aberta $V_{0} \subset V$ de $p$, tal que $\mathcal{W}_{s}\left(p, V_{0}\right)$ é aberto em $\mathcal{W}_{s}(p, \xi)$ com respeito à topologia induzida. Portanto, existe um aberto $V_{1} \subset M$ tal que $\mathcal{W}_{s}\left(p, V_{0}\right)=\mathcal{W}_{s}(p, \xi) \cap V_{1}$. Logo $V_{1}$ é uma vizinhança aberta de $p \operatorname{com} \mathcal{W}_{s}\left(p, V_{0}\right) \subset \mathcal{W}_{s}\left(p, V_{1}\right)$; seja $Z=V_{1} \cap V$,

$$
\begin{aligned}
\mathcal{W}_{s}(p, Z) \subset \mathcal{W}_{s}(p, Z) \cap V_{1} \cap V=\mathcal{W}_{s}\left(p, V_{0}\right) \cap V & =\mathcal{W}_{s}\left(p, V_{0}\right) \\
& \subset \mathcal{W}_{s}\left(p, V_{1}\right) \\
& \subset \mathcal{W}_{s}(p, Z)
\end{aligned}
$$

O que prova que $\mathcal{W}_{s}(p, Z)=\mathcal{W}_{s}(p, \xi) \cap Z$.

$(4) \Rightarrow(5)$. Seja $U$ como no lema (2.2.16). Segue de (4) que é possível achar uma vizinhança aberta $Z \subset U$ de $p$, tal que $\mathcal{W}_{s}(p, Z)=\mathcal{W}_{s}(p, \xi) \cap Z$. Mas $Z \subset U$ implica que 
$\mathcal{W}_{s}(p, Z)$ é uma subvariedade de $M$.

$(5) \Rightarrow(1)$. Seja $V \subset M$ uma vizinhança aberta de $p$ tal que $\mathcal{W}_{s}(p, \xi) \cap V$ é uma subvariedade de $M$. É claro que $\mathcal{W}_{s}(p, \xi)=\bigcup_{t>0} F_{t}^{-1}\left(\mathcal{W}_{s}(p, \xi) \cap V\right)$, como $F_{t}^{-1}$ é um difeomorfismo entre subconjuntos abertos de $M$, temos que $F_{t}^{-1}\left(\mathcal{W}_{s}(p, \xi) \cap V\right)$ é uma subvariedade de $M$ para cada $t \geq 0$. Mas $F_{t}^{-1}\left(\mathcal{W}_{s}(p, \xi) \cap V\right)$ é aberto em $\mathcal{W}_{s}(p, \xi)$ com respeito à topologia induzida, vide a observação (2.2.14). O que prova que $\mathcal{W}_{s}(p, \xi)$ é uma subvariedade de $M$.

Agora é enunciado o teorema de Hartman-Grobman o qual é provado em [10], e simplesmente afirma que um campo vetorial próximo de uma singularidade hiperbólica, é linear.

Definição 2.2.20. Dois campos $\xi \in \mathfrak{X}(M), \beta \in \mathfrak{X}(N)$, são ditos topologicamente equivalêntes ou simplesmente equivalentes, se existir um homeomorfismo $f: M \rightarrow N$ tal que $f$ leva órbitas de $\xi$ em órbitas de $\beta$, e $f^{-1}$ leva órbitas de $\beta$ em órbitas de $\xi$, ou seja, se $\gamma$ é uma curva integral para $\xi$ (resp. para $\beta$ ), então $f \circ \gamma$ é uma curva integral para $\beta$. (resp. $f^{-1}$ o $\gamma$ é uma curva integral para $\xi$ ) Neste caso dizemos que $f$ é uma equivalência topológica entre os campos $\xi$ e $\beta$.

Teorema 2.2.21. (Hartman-Grobman) Seja $\xi$ um campo vetorial de classe $C^{k}$ definido numa variedade $M$, e seja p em $M$ uma singularidade hiperbólica de $\xi$. Então $\xi$ é localmente equivalênte a $\mathrm{d} \xi(p)$ em $p$. Ou seja, existe um aberto $U$ em $M$ contendo $p$, e um aberto $\widetilde{U}$ em $T_{p} M$ contendo a origem tal que $\left.\xi\right|_{U}$ é topologicamente equivalênte ao campo linear $\left.\mathrm{d} \xi(p)\right|_{\vec{U}}$.

Lema 2.2.22. Sejam $\xi \in \mathfrak{X}(M)$, p uma singularidade hiperbólica de $\xi$ e $Z \subset M$ aberto tal que $\mathcal{W}_{u}(p, \xi) \backslash\{p\} \subset Z$. Entäo existe uma vizinhança aberta $V \subset M$ de $p$ tal que para cada $x \in V, x \in \mathcal{W}_{s}(p, \xi)$ ou $t \cdot x \in Z$ para algum $t>0$.

Demonstração. Segue do teorema de Hartman-Grobman que é possível achar vizinhanças abertas $U \subset M$ de $p$ e $\widetilde{U} \subset T_{p} M$ da origem tais que $\left.\xi\right|_{U}$ é topologicamente equivalente a d $\left.\xi(p)\right|_{\widetilde{U}}$. Seja $f: U \rightarrow \widetilde{U}$ uma equivalência topológica entre $\left.\xi\right|_{U}$ e $\left.\mathrm{d} \xi(p)\right|_{\widetilde{U}}$ e fixamos a seguinte notação: $T=\mathrm{d} \xi(p),\left(T_{p} M\right)_{+}=\left(T_{p} M\right)_{+}(T),\left(T_{p} M\right)_{-}=\left(T_{p} M\right)_{-}(T)$ e por $T_{+}, T_{-}$ respectivamente os endomorfismos em $\left(T_{p} M\right)_{+} \mathrm{e}\left(T_{p} M\right)_{-}$obtidos tomando as restrições de $T$. A curva integral de $T$ passando pelo ponto $\left(v_{+}, v_{-}\right) \in T_{p} M=\left(T_{p} M\right)_{+} \oplus\left(T_{p} M\right)_{-}$ em $t=0$ é dada por:

$$
\mathbb{R} \ni t \mapsto\left(e^{t T_{+}} v_{+}, e^{t T_{-}} v_{-}\right) \in\left(T_{p} M\right)_{+} \oplus\left(T_{p} M\right)_{-} .
$$

Tomando uma norma arbitrária em $T_{p} M$ e constantes $\lambda_{+}, \lambda_{-} \in \mathbb{R}$ tais que:

$$
\max _{\lambda \in \mathcal{S}\left(T_{-}\right)} \Re(\lambda)<\lambda_{-}<0<\lambda_{+}<\min _{\lambda \in S\left(T_{+}\right)} \Re(\lambda)
$$


Segue do lema (2.2.9) que é possível achar uma constante positiva $\mathcal{C}$ tal que:

$$
\left\|e^{-t T_{+}}\right\| \leq \mathcal{C} e^{-t \lambda_{+}}, \quad\left\|e^{t T_{-}}\right\| \leq \mathcal{C} e^{t \lambda_{-}}
$$

para todo $t \geq 0$. Ou seja, para $\left(v_{+}, v_{-}\right) \in T_{p} M=\left(T_{p} M\right)_{+} \times\left(T_{p} M\right)_{-}$temos:

$$
\begin{gathered}
\left\|e^{-t T_{+}} v_{+}\right\| \leq \mathcal{C}\left\|v_{+}\right\|, \\
\left\|e^{t T_{-}} v_{-}\right\| \leq \mathcal{C}\left\|v_{-}\right\|,
\end{gathered}
$$

para todo $t \geq 0$. Escolhemos $r>0$ tal que:

$$
B\left(0, r ;\left(T_{p} M\right)_{+}\right) \times B\left(0, r ;\left(T_{p} M\right)_{-}\right) \subset \widetilde{U}
$$

e $r^{\prime}>0 \operatorname{com} r^{\prime}<\frac{r}{\mathcal{C}}$ então as desigualdades (2.27) e (2.28) mostram que a bola fechada $B\left[0, r^{\prime} ;\left(T_{p} M\right)_{+}\right]$(respectivamente $B\left[0, r^{\prime} ;\left(T_{p} M\right)_{-}\right]$) está contida em $\mathcal{W}_{u}\left(0,\left.T\right|_{\tilde{U}}\right)$. (respectivamente $\mathcal{W}_{s}\left(0,\left.T\right|_{\tilde{U}}\right)$ ) Como $f$ leva órbitas de $\xi$ em órbitas de $T$, temos:

$$
f^{-1}\left(B\left[0, r^{\prime} ;\left(T_{p} M\right)_{+}\right]\right) \subset \mathcal{W}_{u}(p, \xi), \quad f^{-1}\left(B\left[0, r^{\prime} ;\left(T_{p} M\right)_{-}\right]\right) \subset \mathcal{W}_{s}(p, \xi) .
$$

Daí, o aberto $f(U \cap Z) \subset T_{p} M$ contém a bola $B\left[0, r^{\prime} ;\left(T_{p} M\right)_{+}\right] \backslash\{0\}$. Como a esfera $S\left[0, r^{\prime} ;\left(T_{p} M\right)_{+}\right]$é compacta, é possível achar $\epsilon>0$ tal que:

$$
S\left[0, r^{\prime} ;\left(T_{p} M\right)_{+}\right] \times B\left(0, \epsilon ;\left(T_{p} M\right)_{-}\right) \subset f(Z \cap U)
$$

Seja $\epsilon^{\prime}>0, \operatorname{com} \epsilon^{\prime} \leq r^{\prime}$ e $\epsilon^{\prime} \leq \frac{\epsilon}{\mathcal{C}}$. Afimação: $V=f^{-1}\left(B\left(0, r^{\prime} ;\left(T_{p} M\right)_{+}\right) \times B\left(0, \epsilon^{\prime} ;\left(T_{p} M\right)_{-}\right)\right)$. Com efeito, dado $x \in V, \operatorname{com} f(x)=\left(v_{+}, v_{-}\right) \in T_{p} M$, i.e., $\left\|v_{+}\right\| \leq r^{\prime}$ e $\left\|v v_{-}\right\| \leq \epsilon^{\prime}$ se $v_{+}=0$, então $f(x) \in B\left[0, r^{\prime} ;\left(T_{p} M\right)_{-}\right] \subset \mathcal{W}_{s}\left(0,\left.T\right|_{\tilde{U}}\right)$, assumimos que $v_{+} \neq 0$, segue da escolha de $\epsilon^{\prime}$ e de (2.28) que:

$$
\begin{aligned}
& \left\|e^{t T_{-}} v_{-}\right\|<r \\
& \left\|e^{t T_{-}} v_{-}\right\|<\epsilon,
\end{aligned}
$$

para cada $t \geq 0$. Também para cada $t \geq 0$, temos:

$$
\left\|e^{t T_{+}} v_{+}\right\| \geq\left\|e^{-t T_{+}}\right\|^{-1}\left\|v_{+}\right\| \geq \frac{1}{\mathcal{C}} e^{t \lambda_{+}}\left\|v_{+}\right\|
$$

mas $v_{+} \neq 0$, então $\lim _{t \rightarrow \infty}\left\|e^{t T_{+}} v_{+}\right\|=+\infty$, como $\left\|v_{+}\right\| \leq r^{\prime}$, existe $t>0$ tal que

$$
\left\|e^{t T+} v_{+}\right\|=r^{\prime}
$$

seja $t_{0}$ o menor $t$, então $\left\|e^{t T_{+}} v_{+}\right\| \leq r^{\prime}<r$ para $0 \leq t \leq t_{0}$, segue de (2.29) e (2.31) que a linha de fluxo (2.26) está em $\widetilde{U}$ para $0 \leq t \leq t_{0}$. i.e.,

$$
\left[0, t_{0}\right] \ni t \mapsto f^{-1}\left(\left(e^{t T_{+}} v_{+}, e^{t T-} v_{-}\right)\right) \in U \subset M
$$


é uma linha de fluxo para $\xi$ passando por $x$. De (2.30) e (2.32), temos:

$$
\left(e^{t_{0} T_{+}} v_{+}, e^{t_{0} T_{-}} v_{-}\right) \in S\left[0, r^{\prime},\left(T_{p} M\right)_{+}\right] \times B\left(0, \epsilon ;\left(T_{p} M\right)_{-}\right) \subset f(Z \cap U),
$$

i.e., que:

$$
f^{-1}\left(\left(e^{t_{0} T_{+}} v_{+}, e^{t_{0} T_{-}} v_{-}\right)\right)=t_{0} \cdot x \in Z
$$

Corolário 2.2.23. Sejam $\xi \in \mathfrak{X}(M)$, p uma singularidade hiperbólica de $\xi$ e $Z \subset M$ aberto tal que $\mathcal{W}_{u}(p, \xi) \backslash\{p\} \subset Z$. Então existe um aberto $V_{0} \subset M$ contendo $\mathcal{W}_{s}(p, \xi)$ tal que para cada $x \in V_{0} \backslash \mathcal{W}_{s}(p, \xi)$ temos $t \cdot x \in Z$ para algum $t>0$.

Demonstração. Seja $V$ como no lema 2.2 .22 e tome $V_{0}=\bigcup_{t \geq 0} F_{t}^{-1}(V)$.

\subsection{Funções de Morse e o Campo Gradiente}

Nesta seção são abordados alguns temas de álgebra linear, é definida a noção de Hessiano de uma aplicação diferenciável num ponto crítico, também definimos o conceito de função de Morse, e se introduz um campo vetorial particular chamado o campo gradiente, o qual é associado a uma função a valores reais definida numa variedade diferenciável; adiante estudaremos os aspectos dinâmicos deste quando a aplicação é uma função de Morse definida numa variedade compacta. Dado um espaço vetorial real $V$, é denotado por $\mathcal{B}(V)$ o espaço das aplicações bilineares definidas em $V, \mathcal{L}\left(\mathbb{R}^{m}, \mathbb{R}^{n}\right)$ denota o espaço das aplicações lineares definidas de $\mathbb{R}^{m}$ em $\mathbb{R}^{n}$.

Definição 2.3.1. Seja $M$ uma variedade diferenciável e seja $f: M \rightarrow \mathbb{R}$ uma aplicação diferenciável. Um ponto $x \in M$ é chamado um ponto crítico de $f$ se $\mathrm{d} f(x)=0$.

$O$ conjunto dos pontos críticos de uma aplicação diferenciável $f$ é denotado por Crit $(f)$; se $a \in \mathbb{R}$, definimos o conjunto dos pontos críticos no nível a como:

$$
\operatorname{Crit}_{a}=\operatorname{Crit}(f) \cap f^{-1}(a)
$$

Definição 2.3.2. Sejam $M$ uma variedade diferenciável de classe $C^{k}$, $p$ um ponto em $M$ e $B: T_{p} M \times T_{p} M \rightarrow \mathbb{R}^{p}$ uma aplicação bilinear. Se $\varphi: U \rightarrow \widetilde{U} \subset \mathbb{R}^{n}$ é uma carta em $M$, $\operatorname{com} p \in U$. Então a aplicação bilinear $\hat{B}: \mathbb{R}^{n} \times \mathbb{R}^{n} \rightarrow \mathbb{R}^{p}$, definida por:

$$
\bar{B}(u, v)=B\left(\mathrm{~d} \varphi(p)^{-1} \cdot u, \mathrm{~d} \varphi(p)^{-1} \cdot v\right), \forall u, v \in \mathbb{R}^{n}
$$

é dita a representação de $B$ na carta $\varphi$. 
Observação 2.3.3. Se $\tilde{B}: \mathbb{R}^{n} \times \mathbb{R}^{n} \rightarrow \mathbb{R}^{p}$ é bilinear e $\varphi: U \rightarrow \widetilde{U} \subset \mathbb{R}^{n}$ carta com $p \in U$, então existe uma única aplicação bilinear $B$ em $T_{p} M$, tal que $\tilde{B}$ representa a $B$ na carta $\varphi$, de fato só basta definir

$$
B(u, v)=\tilde{B}(\mathrm{~d} \varphi(p) \cdot u, \mathrm{~d} \varphi(p) \cdot v)
$$

Lembremos agora a definição de Hessiano no caso de uma função definida no espaço $\mathbb{R}^{m}$, seja $f: U \subset \mathbb{R}^{m} \rightarrow \mathbb{R}^{n}$ uma função diferenciável. A diferencial de $f$, d $f$ é uma aplicação definida em $U$ tomando valores em $\mathcal{L}\left(\mathbb{R}^{m}, \mathbb{R}^{n}\right)$, se tal aplicação é novamente diferenciável num ponto $p \in U$, dizemos que $f$ é duas vezes diferenciável no ponto $p$. Considerando a diferencial de $\mathrm{d} f$ no ponto $p, \mathrm{~d}(\mathrm{~d} f)(p)=\mathrm{d}^{2} f(p): \mathbb{R}^{m} \rightarrow \mathcal{L}\left(\mathbb{R}^{m}, \mathbb{R}^{n}\right)$; como $\mathcal{L}\left(\mathbb{R}^{m}, \mathcal{L}\left(\mathbb{R}^{m}, \mathbb{R}^{n}\right)\right) \simeq \mathcal{B}\left(\mathbb{R}^{m} \times \mathbb{R}^{m}, \mathbb{R}^{n}\right)$ a diferencial segunda ou Hessiano de $f$ no ponto $p$, é a aplicação bilinear $\mathrm{d}^{2} f(p): \mathbb{R}^{m} \times \mathbb{R}^{m} \rightarrow \mathbb{R}^{n}$ definida por:

$$
\mathrm{d}^{2} f(p)(u, v)=\left(\mathrm{d}^{2} f(p) \cdot u\right) \cdot v, \quad \text { para cada } v, u \in \mathbb{R}^{m}
$$

Segue do teorema de Schwarz que o Hessiano assim definido, é uma aplicação bilinear simétrica a qual fica bem difinida em qualquer ponto de $U$; mas no caso de uma função definida numa variedade diferenciável abstrata, não é sempre possivel fazer a definição, como tudo o que é definido em variedades, a idéia é tentar chegar numa definição com a qual o trabalho feito com as cartas não dependa da escolha, o qual neste caso só é verdade quando d $f(p)=0$, ou seja, só é possível definir o Hessiano de uma aplicação $f: M \rightarrow \mathbb{R}$ de classe $C^{2}$, em $p$, se $p$ é um ponto crítico de $f$.

Sejam $f: M \rightarrow \mathbb{R}$ uma aplicação de classe $C^{2}, p \in M$ um ponto e $\varphi: U \rightarrow \widetilde{U}$ uma carta em $M$ com $p \in U$; a composta $f \circ \varphi^{-1}$ é uma aplicação de classe $C^{2}$ definida em $\widetilde{U}$, então é possivel definir a forma bilinear simétrica ou Hessiano de $f \circ \varphi^{-1}$ num ponto $q$ de $\widetilde{U}$. Pela observação (2.3.3) teríamos definida uma forma bilinear simétrica em $T_{p} M$, $B: T_{p} M \times T_{p} M \rightarrow \mathbb{R}$ tal que o Hessiano de $f \circ \varphi^{-1}$ a representa, ou seja, uma forma bilinear dada por:

$$
B=\mathrm{d}^{2}\left(f \circ \varphi^{-1}\right)(\varphi(p)) \circ(\mathrm{d} \varphi(p), \mathrm{d} \varphi(p)),
$$

portanto $B(u, v)=\mathrm{d}^{2}\left(f \circ \varphi^{-1}\right)(\varphi(p)) \circ(\mathrm{d} \varphi(p) \cdot u, \mathrm{~d} \varphi(p) \cdot v) \forall u, v \in T_{p} M$.

Proposição 2.3.4. Seja $M$ uma variedade diferenciável de classe $C^{k}, p \in M$ um ponto crítico de uma aplicação $f: M \rightarrow \mathbb{R}$ de classe pelo menos $C^{2}$. A forma bilinear simétrica definida em (2.33) não depende da escolha da carta $\varphi$, será denotada por $\mathrm{d}^{2} f(p)$ e é chamada o Hessiano de $f$ em $p$.

Demonstração. Sejam $\varphi: U \rightarrow \widetilde{U}$ e $\psi: V \rightarrow \widetilde{V}$ cartas em $M, \operatorname{com} p \in U \cap V$. Para a prova da proposição empregamos os seguintes fatos, se $T$ denota a função de transição entre $\varphi$ e $\psi$, então

$$
\mathrm{d} T(\varphi(p))=\mathrm{d} \psi(p) \circ \mathrm{d} \varphi(p)^{-1}
$$


e dadas aplicações $h$ e $g$, a segunda derivada da composta $h \circ g$ é dada por

$$
\mathrm{d}^{2}(h \circ g)(z)(v, w)=\mathrm{d}^{2} h(g(z)) \circ(\mathrm{d} g(z) v, \mathrm{~d} g(z) w)+\mathrm{d} h(g(z)) \circ \mathrm{d}^{2} g(z)(v, w) .
$$

Então para provar que:

$$
\mathrm{d}^{2}\left(f \circ \varphi^{-1}\right)(\varphi(p)) \circ(\mathrm{d} \varphi(p) v, \mathrm{~d} \varphi(p) w)=\mathrm{d}^{2}\left(f \circ \psi^{-1}\right)(\psi(p)) \circ(\mathrm{d} \psi(p) v, \mathrm{~d} \psi(p) w)
$$

basta aplicar as igualdades (2.34) e (2.35) com $h=f \circ \psi^{-1}, g=\psi \circ \varphi^{-1}$ e $z=\varphi(p)$.

Teorema 2.3.5. Sejam $V$ um espaço vetorial real de dimensão $n$, e $B: V \times V \rightarrow \mathbb{R}$ uma forma bilinear simétrica, então existe uma base $\left\{v_{1}, \cdots, v_{n}\right\}$ de $V$ cujos elementos são ortonormais dois a dois com respeito a $B$ e tal que

$$
B \cong\left(\begin{array}{ccc}
\lambda_{1} & & 0 \\
& \ddots & \\
0 & & \lambda_{n}
\end{array}\right)
$$

em que $\lambda_{i}=b\left(v_{i}, v_{i}\right)$ para cada $i \in\{1, \cdots, n\}$.

O número de entradas positivas na diagonal da matriz do teorema acima é independente da escolha da base, e tal número é chamado o co-índice de $B$; igualmente, o número de entradas negativas e nulas é independente da escolha da base, e tais números são chamados o indice e a nulidade de $B$ respectivamente. No caso em que $B$ tenha nulidade zero, $B$ é dita não-degenerada.

Definição 2.3.6. Um ponto crítico $p \in M$ de uma aplicação diferenciável $f: M \rightarrow \mathbb{R}$ é chamado não-degenerado, se o Hessiano de $f$ em $p, \mathrm{~d}^{2} f(p)$ definido em $T_{p} M$ é nãodegenerado. Uma função diferenciável $f: M \rightarrow \mathbb{R}$ a qual só tem pontos críticos nãodegenerados é dita uma função de Morse.

Definição 2.3.7. O índice de Morse de um ponto crítico $p \in M$, é definido como sendo o índice do Hessiano de $f$ em $p$, e é denotado por $n_{-}(p)$.

Obviamente se o Hessiano de uma aplicação diferenciável $f$ num ponto crítico $p$ é definido positivo ou negativo, então $p$ é não degenerado. A relação entre ponto crítico e o Hessiano é estabelecida assim: Se $p$ é um ponto crítico de uma aplicação diferenciável $f$ de índice zero (resp. de índice dimM), então $p$ é um ponto de mínimo local (resp. máximo local) de $f$. Um ponto crítico não-degenerado $p$ é um ponto de inflexão se, e somente se, o índice de $p$ é positivo e menor que a dimensão de $M$.

Em torno de um ponto crítico não-degenerado de uma aplicação diferenciável $f$, a função pode ser localmente identifcada com um forma quadrática, este é o resultado do seguinte lema, para o qual uma prova pode ser encontrada em [8]. 
Lema 2.3.8. (M. Morse) Seja f uma aplicação real-valuada de classe $C^{k},(k \geq 3)$ definida numa variedade diferenciável arbitraria, e $p \in M$ um ponto crítico não-degenerado de $f$. Então existe uma carta $\varphi: U \rightarrow \widetilde{U} \subset \mathbb{R}^{n}$, com $p \in U$, tal que

$$
f \circ \varphi^{-1}\left(x_{1}, \cdots, x_{n}\right)=f(p)-\left(x_{1}\right)^{2}-\cdots-\left(x_{i}\right)^{2}+\left(x_{i+1}\right)^{2}+\cdots+\left(x_{n}\right)^{2}
$$

em que $i$ é o índice de $p$.

Corolário 2.3.9. Os pontos críticos não-degenerados de uma aplicaçâo $f: M \rightarrow \mathbb{R}$ de classe $C^{k},(k \geq 3)$ são isolados no conjunto Crit $(f)$.

Demonstração. Seja $p$ um ponto crítico não-degenerado de $f$, pelo lema de Morse localmente $f$ tem a forma:

$$
f \circ \varphi^{-1}=f(p)-\left(x_{1}\right)^{2}-\cdots-\left(x_{i}\right)^{2}+\left(x_{i+1}\right)^{2}+\cdots+\left(x_{d}\right)^{2}
$$

em que $i$ é o índice de Morse de $p$. Ou seja, dado $\tilde{p}=\varphi(p) \in \widetilde{U}$, um ponto crítico de $f \circ \varphi^{-1}$ temos que:

$$
\mathrm{d}\left(f \circ \varphi^{-1}\right)(\tilde{p}) \cdot v=2\left\langle\left(-x_{1}, \cdots,-x_{i}, x_{i+1}, \cdots, x_{n}\right), v\right\rangle=0, \quad \text { para cada } v \in \mathbb{R}^{n},
$$

portanto $\tilde{p}=0$.

Observação 2.3.10. No caso em que $M$ é compacta, o conjunto dos pontos críticos nãodegenerados de uma função $f$ definida em $M$ é finito.

É claro que faz sentido perguntar se existem funções de Morse definidas em uma variedade arbitrária? Em [4] pode-se achar o seguinte resultado.

Teorema 2.3.11. Numa variedade diferenciável $M$, sempre é possivel definir funçôes de Morse; além do mais, o conjunto formado pelas funções de Morse definidas em $M$, é um. subconjunto denso no conjunto das funções diferenciáveis em $M$, com respeito à topologia da convergência uniforme.

Definição 2.3.12. Uma métrica Riemanniana numa variedade diferenciável $M$, é uma correspondência $g$, a qual a cada $p$ em $M$, associa um produto interno $g(p)=\langle\cdot, \cdot\rangle_{p}$ definido em $T_{p} M$; além do mais, se $\varphi: U \rightarrow \widetilde{U} \subset \mathbb{R}^{n}$ é uma carta e denotando por $\tilde{g}$ a representação de g na carta $\varphi$, a aplicação:

$$
\tilde{g}: \widetilde{U} \longrightarrow \mathcal{B}\left(\mathbb{R}^{n}\right),
$$

é diferenciável. Para cada $x \in \widetilde{U}$, temos:

$$
\begin{aligned}
\tilde{g}(x)\left(v_{1}, v_{2}\right) & =g\left(\varphi^{-1}(x)\right)\left(\mathrm{d} \varphi^{-1}(x) \cdot v_{1}, \mathrm{~d} \varphi^{-1}(x) \cdot v_{2}\right) \\
& =\left\langle\mathrm{d} \varphi^{-1}(x) \cdot v_{1}, \mathrm{~d} \varphi^{-1}(x) \cdot v_{2}\right\rangle_{\varphi^{-1}(x)}
\end{aligned}
$$


Uma variedade diferenciável $M$ com uma métrica Riemanniana $g$, é chamada uma variedade Riemanniana, e é denotada por $(M, g)$. Uma prova sobre a existência para métricas Riemannianas em variedades aparece em [3].

Definição 2.3.13. Sejam $(M, g)$ uma variedade Riemanniana de classe $C^{k}$ e $f: M \rightarrow \mathbb{R}$ uma aplicação diferenciável. Para cada ponto $p \in M$ consideremos o produto interno \langle\rangle$_{p}$ induzido pela métrica $g$ em $T_{p} M$, definimos o gradiente de $f$ em $p$ como o único vetor $\nabla f(p) \in T_{p} M$, tal que $\mathrm{d} f(p) \cdot v=\langle\nabla f(p), v\rangle_{p}$ para cada $v \in T_{p} M$. A aplicação $\nabla f: M \rightarrow T M$ que a cada $p \in M$, associa $\nabla f(p) \in T_{p} M$ é chamada o campo gradiente de $f$.

Em resumo, dados uma aplicação $f: M \rightarrow \mathbb{R}$ de classe $C^{2}$, um ponto $p \in \operatorname{Crit}(f)$, e uma carta $\varphi: U \rightarrow \widetilde{U}$ em $M$ com $p \in U$, então a definição de Hessiano apresentada anteriormente fornece a seguinte identidade:

$$
\mathrm{d}^{2} f(p)(u, v)=\mathrm{d}^{2}\left(f \circ \varphi^{-1}\right)(\varphi(p))(\mathrm{d} \varphi(p) \cdot u, \mathrm{~d} \varphi(p) \cdot v) .
$$

Também, o ponto $p$ é uma singularidade do campo $\nabla f$, e em (2.14) foi definida a diferencial de um campo numa singularidade, o qual é um endomorfismo linear em $T_{p} M$; a relação existente entre o Hessiano de $f$ em $p$ e a diferencial do campo gradiente $\nabla f$ na singularidade $p$, é dada por:

$$
\mathrm{d}^{2} f(p)\left(v_{1}, v_{2}\right)=g(p)\left(\mathrm{d} \nabla f(p) \cdot v_{1}, v_{2}\right)
$$

Ou seja, $\mathrm{d} \nabla f(p)$ representa o Hessiano de $f$ em $p$ respeito ao produto interno $g(p)$ induzido pela métrica Riemanniana $g$ em $T_{p} M$; pela simetria do Hessiano, segue da observação (2.2.6) e de (2.39) que $\mathcal{S}(\mathrm{d} \nabla f(p)) \subset \mathbb{R}$, logo os pontos críticos não-degenerados de uma aplicação diferenciável $f: M \rightarrow N$, são precisamente as singularidades hiperbólicas do campo $\nabla f$, além do mais, da definição $(2.2 .5)$ e a proposição (2.2.18) segue que $T_{p}\left(\mathcal{W}_{s}(p, \nabla f)\right)=T_{p} M_{-}(\mathrm{d} \nabla f(p))$ portanto, $\operatorname{dim}\left(\mathcal{W}_{s}(p, \nabla f)\right)=n_{-}(p)$

\subsection{Aspectos Dinâmicos do Campo Gradiente}

Nesta seção $f: M \rightarrow \mathbb{R}$ denotará uma aplicação diferenciável definida numa variedade Riemanniana compacta de dimensão $n$. Mas são apresentados alguns resultados no final que não precisam da hipótese de compacidade.

Estamos interesados em estudar o fluxo $F$ do campo $-\nabla f$, o qual como $M$ compacta, é sabido pelo teorema (2.1.8) que é completo. Por comodidade empregaremos a notação $t \cdot x=F(t, x)$ e se $a \in \mathbb{R}, f^{a}$ denota o conjunto $f^{-1}((-\infty, a])$

Se $p \in \operatorname{Crit}(f)$, escreveremos:

$$
\mathcal{W}_{s}(p)=\mathcal{W}_{s}(p,-\nabla f) \text { e } \mathcal{W}_{u}(p)=\mathcal{W}_{u}(p,-\nabla f)
$$


Obviamente, se $p \in M$ é um ponto crítico de $f, t \cdot p=p$ para cada $t \in \mathbb{R}$; caso contrário,

$$
\frac{\mathrm{d} f(t \cdot p)}{\mathrm{d} t}=-\mathrm{d} f(t \cdot p) \cdot \nabla f(t \cdot p)=-\langle\nabla f(t \cdot p), \nabla f(t \cdot p)\rangle_{p}<0
$$

portanto, a aplicação $t \rightarrow f(t \cdot p)$ é estritamente decrescente. Além do mais, para $s, t \in \mathbb{R}$ com $s \leq t$ temos:

$$
f(t \cdot p)=f(s \cdot p)-\int_{s}^{t}|\nabla f(r \cdot p)|^{2} \mathrm{~d} r .
$$

Denotamos por $\mathcal{C}_{x}^{y}$, o conjunto dos caminos diferenciáveis $\sigma:[a, b] \rightarrow M$ tais que $\sigma(a)=x$ e $\sigma(b)=y$, o comprimento de $\sigma \in \mathcal{C}_{x}^{y}$ é dado por:

$$
L(\sigma)=\int_{a}^{b}\left|\sigma^{\prime}(t)\right| \mathrm{d} t
$$

é possível provar vide [6], que para cada $x, y \in M, \mathcal{C}_{x}^{y} \neq \emptyset$; isto justifica a definição de uma distância d em $M$ chamada distância geodésica a qual induz uma topologia em $M$ compatível com a topologia de variedade em $M$, e é definida como:

$$
\mathrm{d}(x, y)=\inf \left\{L(\sigma): \sigma \in \mathcal{C}_{x}^{y}\right\}
$$

Lema 2.4.1. Seja $p$ um ponto crítico isolado de $f$ com $f(p)=c$. Dada uma vizinhança aberta $U \subset M$ de $p$, existem uma vizinhança aberta $V \subset M$ de $p$, e $\epsilon>0$ tais que para cada $y \in V, f(t \cdot y) \geq c-\epsilon$ implica que $t \cdot y \in U$, para cada $t \geq 0$.

Demonstração. Sem perda de generalidade suponhamos que $U \cap \operatorname{Crit}(f)=\{p\}$. Sejam $\rho>0$ tal que $\vec{B}_{\rho}(p) \subset U$, e $C=\{x \in M: \rho / 2 \leq \mathrm{d}(x, p) \leq \rho\}$. É claro que $C \cap C r i t(f)=\emptyset$, segue da compacidade de $M$ que $\delta=\inf \{|\nabla f(x)|: x \in C\}>0$. Seja $V=\bar{B}_{\rho / 2}(p) \cap f^{c+\delta \rho / 4}$, se $x \in V$, é tal que $t \cdot x \notin U$ para um certo $t \geq 0$, então existem reais $0 \leq t_{1} \leq t_{2}$ tais que $t \cdot x \in C$ para $t_{1} \leq t \leq t_{2}, \mathrm{~d}\left(t_{1} \cdot x, p\right)=\rho / 2 \mathrm{e} \mathrm{d}\left(t_{2} \cdot x, p\right)=\rho$. Segue de (2.40) que:

$$
\begin{aligned}
f\left(t_{2} \cdot x\right) & =f\left(t_{1} \cdot x\right)-\int_{t_{1}}^{t_{2}}|\nabla f(t \cdot x)|^{2} \mathrm{~d} r \\
& \leq f(x)-\delta \int_{t_{1}}^{t_{2}}|\nabla f(t \cdot x)| \mathrm{d} r \\
& =f(x)-\delta \mathrm{d}\left(t_{1} \cdot x, t_{2} \cdot x\right) \\
& \leq c+\delta \rho / 4-\delta\left(\mathrm{d}\left(t_{2} \cdot x, p\right)-\mathrm{d}\left(t_{1} \cdot x, p\right)\right) \\
& =c+\delta \rho / 4-\delta \rho / 2 \\
& =f(p)-\delta \rho / 4
\end{aligned}
$$

$\epsilon=\delta \frac{\rho}{4}$ completa a prova.

Lema 2.4.2. Seja $p \in M$. O $\omega$-limite da órbita de $p, \omega(p)$, consiste só de pontos críticos da funçäo $f$. 
Demonstração. Se $y \in \omega(p), y \notin \operatorname{Crit}(f)$, com $f(y)=c$. Então é possível encontrar uma vizinhança $V$ de $y$ em $M$ a qual não contém pontos críticos da $f$ e tal que $S=V \cap f^{-1}(c)$ é uma subvariedade de $M$ com codimensão 1. Além do mais, se $x \in S, T_{x} S$ é ortogonal a $\nabla f(x)$. Considerando a restrição de $F$ sobre $\mathbb{R} \times S, \mathrm{~d} F(0, x): \mathbb{R} \times T_{x} S \rightarrow T_{x} M$ é um isomorfismo para cada $x \in S$, portanto existe abertos $S_{0} \subset S$, com $y \in S_{0}$, e $(-\epsilon, \epsilon) \subset \mathbb{R}$, tal que a aplicação

$$
(t, y) \in(-\epsilon, \epsilon) \times S_{0} \mapsto t \cdot y \in M
$$

é um difeomorfismo sobre algum aberto $U$ em $M$ que contem $y$. Além do mais, existe uma seqüência $\left(t_{n}\right)_{n \geq 1} \mathrm{em} \mathbb{R}$ tal que $\lim _{n \rightarrow+\infty} t_{n}=+\infty$ e $\lim _{n \rightarrow+\infty} t_{n} \cdot p=y$. Então é possível escolher $N \in \mathbb{N}$ tal que para cada $n \geq N, t_{n} \cdot p \in U$, assim existem $t_{1}, t_{2} \in \mathbb{R}$ com $t_{1} \cdot p, t_{2} \cdot p \in U$ e tais que $t_{2} \geq t_{1}+2 \epsilon$, logo é possível achar $y_{1}, y_{2} \in S_{0}$ e $s_{1}, s_{2} \in(-\epsilon, \epsilon)$ tais que $s_{1} \cdot y_{1}=t_{1} \cdot p$, e $s_{2} \cdot y_{2}=t_{2} \cdot p$, portanto temos $\left(t_{1}-s_{1}\right) \cdot p=y_{1} \in S_{0} \subset$ $f^{-1}(c)$ e $\left(t_{2}-s_{2}\right) \cdot p=y_{2} \in S_{0} \subset f^{-1}(c)$; tomando $f, f\left(\left(t_{1}-s_{1}\right) \cdot p\right)=f\left(y_{1}\right)=$ $c$, e $f\left(\left(t_{2}-s_{2}\right) \cdot p\right)=f\left(y_{2}\right)=c$ como a aplicação $t \rightarrow f(t \cdot p)$ é estritamente decrescente, então $\left(t_{1}-s_{1}\right)=\left(t_{2}-s_{2}\right)$ portanto $\left|t_{1}-t_{2}\right|=\left|s_{1}-s_{2}\right|<2 \epsilon$ o qual é uma contradição com o fato que $t_{2} \geq t_{1}+2 \epsilon$.

Lema 2.4.3. Seja $y \in \omega(p)$ para algum $p \in M$, se y é um ponto crítico isolado de $f$, então $\lim _{t \rightarrow \infty} t \cdot p=y$.

Demonstração. Sejam $U$ uma vizinhança de $y,\left(t_{n}\right)_{n \geq 1}$ uma seqüência de números reais tal que $\lim _{n \rightarrow \infty} t_{n}=+\infty$ e $\lim _{n \rightarrow \infty} t_{n} \cdot p=y$. Além do mais, se $c=f(y)$, então $\lim _{n \rightarrow \infty} f\left(t_{n} \cdot p\right)=f\left(\lim _{n \rightarrow \infty} t_{n} \cdot p\right)=f(y)=c$, como $f$ é decrescente ao longo da órbita de $p, f(t \cdot p) \geq c, \forall t \in \mathbb{R}$. Dado $\epsilon>0$, existe $N \in \mathbb{N}$ tal que $n \geq N$, implica que $f\left(t_{n} \cdot p\right) \in B_{\epsilon}(c)$, e para $t \geq t_{n}, c+\epsilon \geq f\left(t_{n} \cdot p\right) \geq f(t \cdot p) \geq c \geq c-\epsilon$, e se $V=U \cap f^{-1}((-\epsilon, \epsilon)), y \in V$; e para cada $t \geq t_{n}, t \cdot p \in V$.

Corolário 2.4.4. Se todos os pontos críticos da $f$ são isolados (em particular se $f$ é uma função de Morse), então cada linha de fluxo de $-\nabla f$ converge a um ponto crítico de $f$, ou seja, se $p \in M$, segue que $\lim _{t \rightarrow+\infty} t \cdot p$ existe e é um ponto crítico de $f$.

Demonstração. De (2.2.4), é claro que $\omega(p) \neq \emptyset$, dos lemas feitos acima segue que se $y \in \omega(p)$, então $y$ é um ponto crítico e pela hipóteses $y$ é isolado, e $\lim _{t \rightarrow+\infty} t \cdot p=y$.

Na seção 2, definimos as variedades invariantes associadas com as singularidades hiperbólicas de um campo vetorial definido numa variedade diferenciável $M$, (não necesariamente compacta) foi provado que tais conjuntos têm estruturas diferenciáveis que os tornam subvariedades imersas de $M$, provaremos agora que no caso do campo gradiente as variedades invariantes são subvariedades mergulhadas de $M$; para isto empregaremos o item 4 da proposição (2.2.19). Os resultados apresentados a continuação não precisam da hipóteses de que $M$ seja compacta. 
Lema 2.4.5. Se $p \in M$ é um ponto crítico não-degenerado de $f: M \rightarrow \mathbb{R} \operatorname{com} f(p)=c$, então dada $V \subset M$ uma vizinhança aberta de $p$, existe $\varphi: Z \rightarrow B\left(0, r ; \mathbb{R}^{k}\right)$ carta em $\mathcal{W}_{s}(p) \operatorname{com} p \in Z \subset V e$

$$
f(x)=c-\|\varphi(x)\|^{2}
$$

para cada $x \in Z$. Além do mais, se $\epsilon \in\left(0, r^{2}\right)$, temos:

$$
f^{-1}([c-\epsilon,+\infty)) \cap \mathcal{W}_{s}(p, \nabla f) \subset Z \subset V .
$$

Em particular, a restriçäo de $\varphi$ a $f^{-1}([c-\epsilon,+\infty)) \cap \mathcal{W}_{s}(p, \nabla f)$ é um homeomorfismo sobre $B\left[0, \sqrt{\epsilon} ; \mathbb{R}^{k}\right]$ que leva $f^{-1}((c-\epsilon)) \cap \mathcal{W}_{s}(p, \nabla f)$ na esfera $S\left[0, \sqrt{\epsilon} ; \mathbb{R}^{k}\right]$.

Demonstração. Suponhamos que $n_{-}(p)=k$, segue da proposição $(2.2 .18)$ que $\mathcal{W}_{s}(p, \nabla f)$ é uma subvariedade imersa de $M$; com $T_{p}\left(\mathcal{W}_{s}(p, \nabla f)\right)$ igual ao auto-espaço negativo do Hessiano de $f$ em $p$, então a restrição $\bar{f}=\left.f\right|_{\mathcal{W}_{s}(p)}$ é diferenciável e $p$ é um ponto crítico não degenerado, pelo lema de Morse, existe uma carta $\varphi: Z \rightarrow B\left(0, r ; \mathbb{R}^{k}\right)$ em $\mathcal{W}_{s}(p, \nabla f)$ tal que:

$$
\tilde{f}(x)=c-\left(\left(\varphi_{1}(x)\right)^{2}+\cdots+\left(\varphi_{k}(x)\right)^{2}\right)=c-\|\varphi(x)\|^{2} .
$$

Seja $\epsilon>0$, com $\epsilon<r^{2}$, provemos que:

$$
f^{-1}([c-\epsilon,+\infty)) \cap \mathcal{W}_{s}(p, \nabla f)=f^{-1}([c-\epsilon, c]) \cap \mathcal{W}_{s}(p, \nabla f)
$$

é um subconjunto de $Z$. Seja $x \in \mathcal{W}_{s}(p, \nabla f)$ com $f(x) \geq c-\epsilon$, suponhamos que $x \notin Z$. Se $\sigma_{x}: I \rightarrow M$ curva integral passando por $x$ associada a $\nabla f, \sigma_{x}: I \rightarrow \mathcal{W}_{s}(p, \nabla f)$, $\left(\mathcal{W}_{s}(p, \nabla f)\right.$ com a topologia de subvariedade imersa) é uma curva integral passando por $x$ associada a $\left.\nabla f\right|_{\mathcal{W}_{s}(p, \nabla f)}$, portanto é contínua e $\lim _{t \rightarrow \infty} \sigma_{x}(t)=p$, daí, para $t$ grande, $\sigma_{x}(t) \in Z$ e $f\left(\sigma_{x}(t)\right)>c-\epsilon$. Logo $\sigma_{x}(t) \in \varphi^{-1}\left(B\left(0, \sqrt{\epsilon}, \mathbb{R}^{k}\right)\right)$, como $\sigma_{x}(0)=x$ não é um elemento de $\varphi^{-1}\left(B\left[0, \sqrt{\epsilon}, \mathbb{R}^{k}\right]\right)$, existe $t>0$ tal que $\sigma_{x}(t) \in Z$ e $\left\|\varphi\left(\sigma_{x}(t)\right)\right\|=\sqrt{\epsilon}$. Logo $f\left(\sigma_{x}(t)\right)=c-\epsilon \leq\left(\sigma_{x}(0)\right)=f(x)$, o que contradiz o fato que $f \circ \sigma_{x}$ é estritamente crescente.

Observação 2.4.6. Se $p \in M$ é um ponto crítico não-degenerado de uma aplicação diferenciável $f: M \rightarrow \mathbb{R}$ com $f(p)=c$ e $n_{-}(p)=k$, como $\mathcal{W}_{s}(p, \nabla f)=\mathcal{W}_{u}(p,-\nabla f)$ dado um valor regular $a=c-\varepsilon$ suficientemente próximo de c, então $\mathcal{W}_{u}(p) \cap f^{-1}(a)$ é difeomorfo a $S^{k-1}$ através da carta $\varphi$ construída no Lema 2.4.5. Além do mais, é possível mostrar que $\mathcal{W}_{s}(p, \nabla f)=\mathcal{W}_{u}(p,-\nabla f)$ é homeomorfo a $\mathbb{R}^{k}$. Com efeito, definimos um homeomorfismo $\bar{\varphi}: \mathcal{W}_{s}(p, \nabla f) \rightarrow \mathbb{R}^{k}$ fazendo:

$$
\bar{\varphi}(x)= \begin{cases}\frac{1}{\sqrt{\epsilon}} \varphi(x), & \text { se } x \in \mathcal{W}_{s}(p, \nabla f) \cap f^{-1}([a, c]) \\ \frac{1-t}{\sqrt{\epsilon}} \varphi(y), & \text { se } x=t \cdot y \operatorname{com} y \in \mathcal{W}_{s}(p, \nabla f) \cap f^{-1}(a) \text { e } t \leq 0\end{cases}
$$


Teorema 2.4.7. Seja $f: M \rightarrow \mathbb{R}$ uma aplicaçăo diferenciável e $p \in M$ um ponto crítico não-degenerado de $f$. Entäo $\mathcal{W}_{s}(p, \nabla f)$ (que é igual a $\mathcal{W}_{u}(p,-\nabla f)$ ) é subvariedade conexa de $M$, cujo espaço tangente no ponto $p$ é igual o auto-espaço negativo do endomorfismo linear em $T_{p} M$ que representa o Hessiano de $f$ no ponto $p$.

Demonstração. Seja $V \subset M$ uma vizinhança aberta de $p, c=f(p)$. Escolha $\epsilon>0$ como no lema acima, se $Z=f^{-1}((c-\epsilon,+\infty)) \cap V$, é claro que $\mathcal{W}_{s}(p, Z) \subset \mathcal{W}_{s}(p) \cap Z$, se $x \in \mathcal{W}_{s}(p) \cap Z$, dada $\sigma_{x}$ curva integral maximal passando por $x, f \circ \sigma_{x}$ cresce ao longo das linhas de fluxo, e $f \circ \sigma_{x}(0)=f(x) \operatorname{com} x \in Z$, daí $f(x) \geq c-\epsilon$, e para cada $t \geq 0$, $f \circ \sigma_{x}(t) \geq f(x) \geq c-\epsilon$; i.e., para cada $t>0, \sigma_{x}(t) \in Z$. Mas $\lim _{t \rightarrow \infty} \sigma_{x}(t)=p$, então como $f$ é contínua, $\lim _{t \rightarrow \infty} f\left(\sigma_{x}(t)\right)=c$; portanto $x \in \mathcal{W}_{s}(p)$ e $\mathcal{W}_{s}(p) \cap Z=\mathcal{W}_{s}(p, Z)$. 


\section{Capítulo 3}

\section{O Complexo de Morse-Witten}

Neste capítulo o principal objetivo é definir o complexo de Morse-Witten associado a uma função de Morse definida numa variedade diferenciável compacta de dimensão $n$.

\subsection{Orientação e Transversalidade}

Nesta seção nossa referência básica para notação e terminologia é [12], além do mais todos os resultados, inclusive as demostrações. Nosso objetivo é empregando topologia diferencial introduzir alguns conceitos os quais permitem a definição do complexo de Morse-Witten.

Sejam $V$ um espaço vetorial real de dimensão $n$, e $\beta$ uma base ordenada para $V$. Dada $\alpha$ outra base ordenada para $V$, então $\beta$ é dita equivalentemente orientada a $\alpha$, se a matriz de mudança de base de $\beta$ para $\alpha$ tem determinante positivo. É claro que a relação acima é de equivalência, e gera uma partição no conjunto das bases ordenadas de $V$ em duas classes $\mathcal{C}_{1}$ e $\mathcal{C}_{2}$. Uma orientação em $V$, é ema decisão arbitrária de fixar um sinal positivo $(+1)$, numa das classes de equivalência em $V$ dadas acima, e um sinal negativo (-1) na outra. Fica claro então que um espaço vetorial real, admite só duas orientações. O sinal de uma base ordenada $\beta$, é o sinal da classe à qual pertence; e é chamado sua orientação; ou seja, a base $\beta$ é orientada positiva ou negativa, depende da orientação escolhida para $V$. No espaço Euclidiano, a orientação para a qual a base canônica tem sinal positivo, é chamada orientação standard. A ordem das bases é importante, pois a troca de dois elementos numa base ordenada, produz uma nova base com um ordem diferente e com orientação oposta. No caso de dimensão zero, é conveniente definir uma orientação com a escolha de um sinal +1 ou -1 .

Se $T: V \rightarrow W$ é um isomorfismo entre espaços vetoriais reais, sempre que duas 
bases ordenadas $\beta$ e $\alpha$ para $V$ estejam na mesma classe de equivalência, segue do fato que a matriz de mudança de bases de $T \beta$ para $T \alpha$ coincide com a matriz mudança de base de $\beta$ para $\alpha$, que $T \beta$ e $T \alpha$ são equivalentemente orientadas; portanto, se $V$ e $W$ são orientados, quer dizer se é especificada uma orientação em ambos, $T \beta$ sempre tem a mesma orientação de $\beta$ ou sempre tem orientação oposta; ou seja, $T$ preserva orientação, ou $T$ reverte orientação. Um isomorfismo que preserva orientação, é dito isomorfismo positivo. Com a noção de orientação num espaço vetorial real, é possível definir tal noção no caso de uma variedade diferenciável.

Definição 3.1.1. Sejam $M$ uma variedade diferenciável, e $x$ um ponto em $M$. Uma orientação para $M$ em x é uma orientação para o espaço vetorial $T_{x} M$.

Definição 3.1.2. Uma variedade diferenciável $M$ é dita orientável, se é possível associar uma orientação em cada ponto $x \in M$ a qual depende continuamente de $x$. i.e., $M$ é orientável se é possível escolher para cada $x \in M$ uma orientação para o espaço vetorial $T_{x} M$, a qual depende continuamente de $x$. Ou seja, para cada $x \in M$, existe uma carta $\varphi: U \rightarrow \widetilde{U} \mathrm{em} M \operatorname{com} x \in U$, a qual preserva orientação no sentido que para cada $y \in U$, o isomorfismo $\mathrm{d} \varphi(y): T_{y} M \rightarrow \mathbb{R}^{n}$ é positivo; em que é subentendido que $\mathbb{R}^{n}$ tem a orientação standard. A particular escolha das orientações para cada $x \in M$, é chamada uma orientação para $M$; uma variedade diferenciável dotada com uma orientação é dita orientada.

Uma aplicação entre variedades diferenciáveis com a propriedade da carta na definição acima, é dita positiva. É claro que uma orientação numa variedade diferenciável orientável de dimensão zero, pode ser entendida como uma função $\tau: M \rightarrow\{-1,1\}$.

Se $N \subset M$ é uma subvariedade de dimensão $k$ então uma carta de subvariedade para $N$ é uma carta $\varphi: U \rightarrow \widetilde{U} \subset \mathbb{R}^{n}$ em $M$ tal que $\varphi(U \cap N)=\widetilde{U} \cap\left(\mathbb{R}^{k} \times\{0\}^{n-k}\right)$. Se $\varphi$ é uma carta de subvariedade para $N$ então para todo $x \in N$ temos que d $\varphi(x): T_{x} M \rightarrow \mathbb{R}^{n}$ induz um isomorfismo entre $T_{x} M / T_{x} N$ e $\mathbb{R}^{n} / \mathbb{R}^{k} \simeq \mathbb{R}^{n-k}$.

Definição 3.1.3. Seja $N \subset M$ uma subvariedade de dimensão $k$ de uma variedade diferenciável $M$. Dizemos que $N$ é transversalmente orientada, ou que $N$ tem uma orientação transversa, se para cada $x \in N$, temos uma orientação no espaço $T_{x} M / T_{x} N$ de modo que podemos cobrir $N$ por cartas de subvariedade $\varphi: U \rightarrow \widetilde{U} \subset \mathbb{R}^{n}$ tal que para todo $x \in U$ o isomorfismo de $T_{x} M / T_{x} N$ sobre $\mathbb{R}^{n-k}$ induzido por $\mathrm{d} \varphi(x)$ é positivo.

Temos a seguinte:

Proposição 3.1.4. Se $M$ é uma variedade diferenciável conexa e orientável então $M$ admite exatamente duas orientações; além do mais, toda variedade simplesmente conexa é orientável. Similarmente, se $N$ é uma subvariedade conexa transversalmente orientável de uma variedade diferenciável $M$ então $N$ admite exatamente duas orientações transversas; além do mais, se $N$ é simplesmente conexa então $N$ é transversalmente orientável. 
Antes de passar a falar de transversalidade, é conveniente a seguinte observação:

Observação 3.1.5. Se $V$ é a soma direta de dois subespaços $V_{1}$ e $V_{2}$, uma orientação em qualquer par deles induz uma orientação no terceiro, assim se $\beta_{1}, \beta_{2}$ bases ordenadas para $V_{1}$ e $V_{2}$ respectivamente, definimos o sinal de $\beta=\left(\beta_{1}, \beta_{2}\right)$ como o produto dos sinais de $\beta_{1}$ e $\beta_{2}$. Mais geralmente, consideremos a seqüência exata curta de espaços vetoriais:

$$
0 \longrightarrow A \stackrel{\varphi}{\longrightarrow} B \stackrel{\psi}{\longrightarrow} C \longrightarrow 0
$$

uma orientação em qualquer par dos espaços induz uma orientaçào no terceiro. Se $\left\{a_{1}, \ldots, a_{n}\right\},\left\{c_{1}, \ldots, c_{r}\right\}$ são bases ordenadas para $A$ e $C$ respectivamente, tomamos $\left\{\varphi\left(a_{1}\right), \ldots, \varphi\left(a_{n}\right), u_{1}, \ldots, u_{r}\right\}$ base para $B$, em que $\psi\left(u_{i}\right)=c_{i}$. Daí, se $V$ é um espaço vetorial real de dimensấo finita, e $V=V_{1}+V_{2}$, então orientações em $V_{1}$ e em $V / V_{2}$, induzem uma orientação na interseção $V_{1} \cap V_{2}$. Basta só considerar a seqüência exata curta:

$$
0 \longrightarrow V_{1} \cap V_{2} \stackrel{i}{\longrightarrow} V_{1} \stackrel{q}{\longrightarrow} V / V_{2} \longrightarrow 0
$$

em que $i$ e q denotam a aplicação inclusão e quociente respectivamente.

Convenção 3.1.6. Para cada $n \geq 0$, a orientação diferenciável em $S^{n}$ determinada pelo vetor normal que aponta para fora é definida como segue; para $n=0$ simplesmente tomamos um sinal + no ponto $1 \in S^{0}$ e um sinal - no ponto $-1 \in S^{0}$; se $n \geq 1$, para cada $x \in S^{n}$, orientamos o espaço vetorial $T_{x} S^{n}$ de tal forma que $\left(x, v_{1}, \ldots v_{n}\right)$ seja uma base orientada positiva para $\mathbb{R}^{n+1}$, para cada base orientada positiva $\left(v_{1}, \ldots, v_{n}\right)$ para $T_{x} S^{n}$. No restante do texto, é assumido que esferas em qualquer dimensão têm a orientação diferenciável determinada pelo vetor normal que aponta para fora.

Agora passamos a falar de transversalidade, é sabido que se $f: M \rightarrow N$ é uma aplicação diferenciável, e $y$ é um valor regular, então $f^{-1}(y)$ é uma subvariedade de $M$ com dimensão igual a $\operatorname{dim} M-\operatorname{dim} N$, e para cada $x \in f^{-1}(y), T_{x} f^{-1}(y)=\operatorname{Ker}(\mathrm{d} f(x))$; se é assumido que $P$ é uma subvariedade de $N$, e considerando a relação $f(x) \in P$. Quando é possível ter uma estrutura de variedade no conjunto de soluções da relação? Para resolver esta questão, precisamos definir uma nova propriedade diferencial, a qual é uma extensão da noção de regularidade.

Definição 3.1.7. Sejam $M, N$ e $P$ variedades diferenciáveis, $f: M \rightarrow N, g: P \rightarrow N$ aplicações diferenciáveis, com $g$ mergulho. Então dizemos que $f$ e $g$ são transversais $(f \pitchfork g)$, se sempre que $f(x)=g(y)$, as imagens das diferenciais $\mathrm{d} f(x): T_{x} M \rightarrow T_{f(x)} N$, $\mathrm{d} g(y): T_{y}(P) \rightarrow T_{g(y)} N$, geram $T_{f(x)} N$.

Da definição acima temos como caso particular o seguinte, se $M$ e $P$ são subvariedades de uma variedade $N$, dizemos que $M$ e $P$ são transversais em $N$, se as respectivas inclusōes $i_{M}: M \rightarrow N$ e $i_{P}: P \rightarrow N$ são transversais. 
Teorema 3.1.8. Na situação da definição (3.1.7), com $\operatorname{dim} M=m, \operatorname{dim} N=n e$ $\operatorname{dim} P=p$, então $f^{-1}(f(M) \cap g(P))$ é uma subvariedade de $M$, com dimensão $m+p-n$.

Corolário 3.1.9. Se $M$ e $P$ são subvariedades transversais de uma variedade diferenciável $N$, então a interseção $M \cap P$ é uma subvariedade de $M$ e portanto de $N$, além do mais, $\operatorname{dim}(M \cap P)=\operatorname{dim} M+\operatorname{dim} P-\operatorname{dim} N$.

\section{Observação 3.1.10.}

- Se $M$ e $P$ transversais, se $x \in M \cap P$, então o espaço tangente a $x$ em $N$, é gerado pelos subespaços $T_{x} P$ e $T_{x} M$; também é claro que $T_{x}(M \cap P) \subset T_{x} M \cap T_{x} P$; e pela transversalidade e o corolário acima segue que $n=p+m-\operatorname{dim}\left(T_{x} M \cap T_{x} P\right)$, ou seja $\operatorname{dim}\left(T_{x} M \cap T_{x} P\right)=\operatorname{dim}(M \cap P)=\operatorname{dim} T_{x}(M \cap P), \operatorname{logo} T_{x} M \cap T_{x} P=T_{x}(M \cap P)$.

- Se $f: M \rightarrow N$ é uma submersão, então $f \pitchfork P$ para toda subvariedade $P$ de $N$.

- Uma condição suficiente para que a soma das definições anteriores seja direta é que a codimensão de $P$ em $N$, seja igual à dimensão de $M$. Quer dizer se $P$ é uma subvariedade de uma variedade diferenciável $N$, com codimensão igual à dimensão de $M$, e $f: M \rightarrow N$ é uma aplicação diferenciável transversal a $P$, então se $f(x) \in P$, $f$ é uma imersấo em $x$ e $T_{f(x)} N=T_{f(x)} P \oplus \operatorname{Im}(\mathrm{d} f(x))$.

Por último definimos outro conceito da topologia diferencial. Se $f: M \rightarrow N$ é uma aplicação diferenciável com $M$ variedade diferenciável orientada compacta. Se $P \subset N$ é uma subvariedade fechada de $N$, com uma orientação transversa, tal que $f \pitchfork P$ e $\operatorname{Cod}_{N}(P)=\operatorname{dim} M$. Então, segue da observação (3.1.10), que se $f(x) \in P, f$ é uma imersão em $x$ e $T_{f(x)} N=T_{f(x)} P \oplus \operatorname{Im}(\mathrm{d} f(x))$. Além do mais, do corolário (3.1.9) temos que $f^{-1}(P)$ é uma subvariedade de $M$ de dimensão zero, portanto é um subconjunto discreto, logo da compacidade temos que é finito. Para cada $x \in f^{-1}(P)$, associamos um sinal como segue: $\operatorname{sinal}(x)=+1$, se o isomorfismo $T_{x} M \stackrel{\pi \mathrm{d} f(x)}{\longrightarrow} T_{f(x)} N / T_{f(x)} P$ é positivo; caso contrário, $\sin a l(x)=-1$.

Definição 3.1.11. Sejam $M$ uma variedade orientada compacta, e $f: M \rightarrow N$ uma aplicação diferenciável. Se $P \subset N$ é uma subvariedade fechada de $N$, orientada transversalmente tal que $f \pitchfork P$ e $\operatorname{Cod}_{N}(P)=\operatorname{dim} M$. Então definimos o número de interseção no sentido diferenciável de $f$ com $P$ como sendo o inteiro:

$$
\eta(f, P)_{d}=\sum_{x \in f^{-1}(P)} \operatorname{sinal}(x)
$$

Observação 3.1 .12 .

- No caso em que $\operatorname{Cod}_{N}(P)=\operatorname{dim} M=0$, uma orientação transversa em $P$, é entendida como a escolha de um sinal em cada componente conexa de $N$; e neste caso, o 
sinal de um ponto $x \in f^{-1}(P)$ é positivo, se o sinal da componente conexa a qual pertenece $f(x)$ coincide com o sinal do ponto $x$ dado pela orientação da variedade de dimensão zero $M$. Caso contrário, o sinal de $x$ é negativo.

- A definição de número de interseção pode ser feita de forma mais geral, sem precisar da hipóteses que a função $f$ seja diferenciável, basta só com a hipóteses de continuidade; mas tal definição envolve o fato que o número de interseçâo no sentido diferenciável é um invariante homotópico. Vide [12] e Seção 4.2 adiante.

- Como caso particular do conceito de número de interseção quando a subvariedade é um ponto, e a função é definida entre variedades de igual dimensão, obtém-se a noção de grau de uma aplicação continua. Vide [12].

\subsection{Transversalidade das Variedades Invariantes}

Nesta seção contiuamos o estudo da dinâmica do campo gradiente com algumas suposições sobre transversalidade das variedades invariantes pelo fluxo associado a tal campo.

$f: M \rightarrow \mathbb{R}$, denota uma função de Morse definida numa variedade Riemanniana compacta de dimansão $n$.

Dados pontos críticos $p, q \in M$, uma linha de fluxo de $p$ para $q$ é definida como uma curva integral para $-\nabla f, \sigma: \mathbb{R} \rightarrow M$ tal que $\lim _{t \rightarrow+\infty} \sigma(t)=q$ e $\lim _{t \rightarrow-\infty} \sigma(t)=p$; em outras palavras, $\sigma: \mathbb{R} \rightarrow M$ é uma curva integral para $-\nabla f$ cuja imagem está contida na interseçāo $\mathcal{W}_{u}(p) \cap \mathcal{W}_{s}(q)$. Se $p \in \operatorname{Crit}(f)$, como o conjunto $\mathcal{W}_{u}(p)$ é invariante pelo fluxo de $-\nabla f, \mathcal{W}_{u}(p)$ é uma união de linhas de fluxo de $-\nabla f$. Em particular, para $x \in \mathcal{W}_{u}(p)$ temos:

$$
-\nabla f(x)=\left.\frac{\mathrm{d}}{\mathrm{d} t}(t \cdot x)\right|_{t=0} \in T_{x}\left(\mathcal{W}_{u}(p)\right)
$$

Além do mais, como $f$ é estritamente decrescente ao longo das linhas de fluxo não constantes de $-\nabla f$, então $p$ é um máximo global de $\left.f\right|_{\mathcal{W}_{u}(p)}$ e um mínimo global de $\left.f\right|_{\mathcal{W}_{s}(p)}$. Portanto, $\mathcal{W}_{u}(p) \cap \mathcal{W}_{s}(p)=\{p\}$.

Se $p, q \in \operatorname{Crit}(f)$, e assumimos que as variedades $\mathcal{W}_{u}(p)$ e $\mathcal{W}_{s}(q)$ são transversais e não disjuntas, segue de (2.4.7) e (3.1.9) que a interseção $\mathcal{W}_{u}(p) \cap \mathcal{W}_{s}(q)$ é uma subvariedade de $M$ com dimensão $n_{-}(p)-n_{-}(q)$, Além do mais, de (3.1.10) temos que para cada $x \in$ $\mathcal{W}_{u}(p) \cap \mathcal{W}_{s}(q)$

$$
T_{x}\left(\mathcal{W}_{u}(p) \cap \mathcal{W}_{s}(q)\right)=T_{x}\left(\mathcal{W}_{u}(p)\right) \cap T_{x}\left(\mathcal{W}_{s}(q)\right)
$$

em particular, de (3.1), $-\nabla f(x) \in T_{x}\left(\mathcal{W}_{u}(p) \cap \mathcal{W}_{s}(q)\right)$. Temos provado o seguinte resultado:

Corolário 3.2.1. Se p, q são pontos críticos distintos de f. Assumindo que as variedades $\mathcal{W}_{u}(p)$ e $\mathcal{W}_{s}(q)$ são transversais e não disjuntas, entäo $n_{-}(p)>n_{-}(q)$. 
Corolário 3.2.2. Sejam $p$, q pontos críticos distintos de $f$, assumindo que as variedades $\mathcal{W}_{u}(p)$ e $\mathcal{W}_{s}(q)$ são transversais e não disjuntas. Seja $a \in \mathbb{R}$ um valor regular de $f$ tal que $\mathcal{W}_{u}(p) \cap \mathcal{W}_{s}(q) \cap f^{-1}(a) \neq \emptyset$. Então, a interseção $\mathcal{W}_{u}(p) \cap \mathcal{W}_{s}(q) \cap f^{-1}(a)$, é subvariedade de $M$ com dimensão $n_{-}(p)-n_{-}(q)-1$, e para cada $x \in \mathcal{W}_{u}(p) \cap \mathcal{W}_{s}(q) \cap f^{-1}(a)$, o espaço tangente em $x$ é dado por:

$$
T_{x}\left(\mathcal{W}_{u}(p) \cap \mathcal{W}_{s}(q) \cap f^{-1}(a)\right)=T_{x}\left(\mathcal{W}_{u}(p)\right) \cap T_{x}\left(\mathcal{W}_{s}(q)\right) \cap \nabla f(x)^{\perp}
$$

Demonstração. É claro que $f^{-1}(a)$ é uma subvariedade de $M$ com codimensão 1 , e para cada $x \in f^{-1}(a), T_{x} f^{-1}(a)=\nabla f(x)^{\perp}$; de $(3.1), \nabla f(x) \in T_{x}\left(\mathcal{W}_{u}(p) \cap \mathcal{W}_{s}(q)\right)$ portanto $f^{-1}(a)$ e $\mathcal{W}_{u}(p) \cap \mathcal{W}_{s}(q)$ são transversais, o resultado segue do corolário (3.1.9).

Definição 3.2.3. Dado um inteiro $k \in \mathbb{Z}$, dizemos que $f: M \rightarrow \mathbb{R}$ satisfaz a condição de Morse-Smale de ordem $k$ se para cada par de pontos críticos $p, q \in M$ com $n_{-}(p)-n_{-}(q) \leq$ $k$, as variedades $\mathcal{W}_{u}(p)$ e $\mathcal{W}_{s}(q)$ são transversais (possivelmente disjuntas). Se $f$ satisfaz a condição de Morse-Smale de ordem $k$ para cada inteiro $k$, dizemos simplesmente que $f$ satisfaz a condição de Morse-Smale.

Observamos que se $f: M \rightarrow \mathbb{R}$ satisfaz a condição de Morse-Smale de ordem 0, dados pontos críticos distintos $p$ e $q$ de $f$, com $n_{-}(p) \leq n_{-}(q)$, então $\mathcal{W}_{u}(p) \cap \mathcal{W}_{s}(q)=\emptyset$. i.e., o índice dos pontos críticos decresce quando andamos ao longo de uma linha de fluxo de $-\nabla f$, também se $n_{-}(p)-n_{-}(q)=1$, e as variedades $\mathcal{W}_{u}(p), \mathcal{W}_{s}(q)$ são transversais e não disjuntas, então a variedade $\mathcal{W}_{u}(p) \cap \mathcal{W}_{s}(q)$ tem dimensão 1 , e consiste de uma coleção de linhas de fluxo de $-\nabla f$ ligando $p$ com $q$. Na verdade, é possível mostrar que o número de linhas ligando $p \operatorname{com} q$ é finito. Além do mais, para cada $x \in \mathcal{W}_{u}(p) \cap \mathcal{W}_{s}(q)$, temos que $T_{x}\left(\mathcal{W}_{u}(p) \cap \mathcal{W}_{s}(q)\right)$ é gerado pelo vetor $-\nabla f(x)$. Vejamos pois que com as hipóteses acima o conjunto $\mathcal{W}_{u}(p) \cap \mathcal{W}_{s}(q)$ é só uma coleção finita de linhas de fluxo para o campo $-\nabla f$. Para fazer isto, precisamos de alguns lemas preparatórios.

Lema 3.2.4. Seja $p \in \operatorname{Crit}(f)$. Se $x \in \overline{\mathcal{W}_{s}(p)}$, então para cada $t \in \mathbb{R}, t \cdot x \in \overline{\mathcal{W}_{s}(p)}$. Em particular, pela continuidade de $f, f(t \cdot x) \geq f(p)$ para cada $t \in \mathbb{R}$.

Demonstração. Dado $t \in \mathbb{R}$, como $F_{t}$ é continua e $F_{t}\left(\mathcal{W}_{s}(p)\right) \subset \mathcal{W}_{s}(p)$, então

$$
F_{t}\left(\overline{\mathcal{W}_{s}(p)}\right) \subset \overline{F_{t}\left(\mathcal{W}_{s}(p)\right)} \subset \overline{\mathcal{W}_{s}(p)}
$$

Lema 3.2.5. Seja $p \in$ Crit $_{c}$. Então, $\overline{\mathcal{W}_{s}(p)} \cap f^{-1}(c)=\{p\}$

Demonstração. Seja $x \in \overline{\mathcal{W}_{s}(p)}$ tal que $f(x)=c$. Vejamos que $x=p$. Pelo lema 3.2.4, para cada $t \in \mathbb{R}$, temos $f(t \cdot x) \geq f(p)=c$; por outro lado, $f$ decresce ao longo das linhas de fluxo de $-\nabla f$, portanto para $t \geq 0, f(t \cdot x) \leq f(x)=c$; ou seja, $f(t \cdot x)=c$ 
para todo $t \geq 0$; daí, obtemos que $x$ é um ponto crítico de $f$ veja corolário (2.4.4). Suponhamos que $x \neq p$, como $M$ Hausdorff, existem abertos disjuntos $U_{x}$ e $U_{p}$ contendo $x$ e $p$ respectivamente; segue do lema (2.4.1) que existem um aberto $V, x \in V \subset U_{x} \subset M$ e $\epsilon>0$, tais que para cada $y \in V$,

$$
f(t \cdot y) \geq c-\epsilon \Longrightarrow t \cdot y \in U_{x}
$$

como $x \in \overline{\mathcal{W}_{s}(p)}, \emptyset \neq V \cap \mathcal{W}_{s}(p) \subset U_{x} \cap \mathcal{W}_{s}(p)$. Se $y \in V \cap \mathcal{W}_{s}(p)$, para cada $t \in \mathbb{R}$ temos que $t \cdot y \in \mathcal{W}_{s}(p)$ e $f(t \cdot y) \geq f(p)=c \geq c-\epsilon$, portanto, de (3.3) segue que:

$$
t \cdot y \in U_{x}, \text { para cada } t \in \mathbb{R}
$$

além do mais,

$$
\lim _{t \rightarrow+\infty} t \cdot y=p
$$

mas (3.4) e (3.5), contradizem o fato que $U_{x}$ e $U_{p}$ são disjuntos.

Lema 3.2.6. Dados pontos críticos distintos $p, q \in \operatorname{Crit}(f)$. Então $q \in \overline{\mathcal{W}_{s}(p)}$ se, e somente se, existe $x \in \mathcal{W}_{u}(q) \backslash\{q\}$, tal que $x \in \overline{\mathcal{W}_{s}(p)}$.

Demonstração. Se existe $x \in \mathcal{W}_{u}(q) \backslash\{q\}$, tal que $x \in \overline{\mathcal{W}_{s}(p)}$, segue do lema (3.2.4) que para cada $t \in \mathbb{R}, t \cdot x \in \overline{\mathcal{W}_{s}(p)}$. Em particular $\lim _{t \rightarrow-\infty} t \cdot x=q \in \overline{\mathcal{W}}_{s}(p)$. Reciprocamente suponhamos que $q \in \overline{\mathcal{W}_{s}(p)}$, se não existe $x \in \mathcal{W}_{u}(q) \backslash\{q\}$ tal que $x \in \overline{\mathcal{W}_{s}(p)}$, então $Z=M \backslash \overline{\mathcal{W}_{s}(p)}$ é um aberto que contém $\mathcal{W}_{u}(q) \backslash\{q\}$, pelo lema (2.2.22) existe um aberto $V$ em $M$ que contém $q$ e tal que para cada $y \in V$ temos: $y \in \mathcal{W}_{s}(q)$ ou $t \cdot y \in Z$ para algum $t>0$. Como $q \in \overline{\mathcal{W}_{s}(p)}$, e $p$ distinto de $q$, existe $y \in V \cap \mathcal{W}_{s}(p)$, tal que $y \notin \mathcal{W}_{s}(q)$, portanto $t \cdot y \in Z$ para algum $t>0$, o qual contradiz o fato que $t \cdot y \in \mathcal{W}_{s}(p)$.

Definição 3.2.7. Uma linha de fluxo por passos é uma seqüência $\gamma=\left(\gamma_{1}, \ldots, \gamma_{k}\right)$ de linhas de fluxo $\gamma_{i}: \mathbb{R} \rightarrow M$ para $-\nabla f$ tais que $\lim _{t \rightarrow+\infty} \gamma_{i}(t)=\lim _{t \rightarrow-\infty} \gamma_{i+1}(t)$ para cada $i=1, \ldots, k-1$. Dizemos que $k$ é o número de passos de $\gamma$ ou que $\gamma$ é a $k$-step linha de fluxo. Se $p=\lim _{t \rightarrow-\infty} \gamma_{1}(t)$ e $q=\lim _{t \rightarrow+\infty} \gamma_{k}(t)$, então dizemos que $\gamma$ é a k-step linha de fluxo de p para $q$. No caso em que $p=q$, é claro que existe uma linha de fluxo por passos de $p$ para $q \operatorname{com} k=0$.

Se $f$ satisfaz a condição de Morse- Smale de ordem 1 e se existe uma $k$-step linha de fluxo de $p \in \operatorname{Crit}(f)$ para $q \in \operatorname{Crit}(f) \operatorname{com} k \geq 1$, então $n_{-}(p)-n_{-}(q) \geq k$. Dados $p, q \in \operatorname{Crit}(f)$, então obviamente $\mathcal{W}_{s}(q) \cap \mathcal{W}_{u}(p)$ é não vazio se, e somente se, existe uma 1-step linha de fluxo de $p$ para $q$.

Lema 3.2.8. Seja $q \in \operatorname{Crit}(f)$. Se $x \in \overline{\mathcal{W}_{s}(q)}$, então existe uma linha de fluxo por passos de $q_{1}=\lim _{t \rightarrow+\infty} t \cdot x \in \operatorname{Crit}(f)$ para $q$. 
Demonstração. Segue do lema (3.2.4) que $q_{1} \in \overline{\mathcal{W}_{s}(q)}$. Se $q=q_{1}$, pronto. Caso cotrario, pelo lema (3.2.6) existe $x_{1} \in \mathcal{W}_{u}\left(q_{1}\right) \backslash\left\{q_{1}\right\}$, com $x_{1} \in \overline{\mathcal{W}_{s}(q)}$. Seja $q_{2}=\lim _{t \rightarrow+\infty} t \cdot x_{1} \epsilon$ Crit $(f)$. Observamos que existe uma linha de fluxo de $-\nabla f$ de $q_{1}$ para $q_{2}$ e $f\left(q_{2}\right)<f\left(q_{1}\right)$. Além do mais, $q_{2} \in \overline{\mathcal{W}_{s}(q)}$. Se $q_{2}=q$ pronto. Caso contrário, podemos continuar inditivamente até algum $q_{n}=q$; caso contrário, obtém-se uma seqüência de pontos críticos $\left(q_{n}\right)_{n \geq 1} \operatorname{com} f\left(q_{1}\right)>f\left(q_{2}\right)>\cdots$, o que contradiz o fato que o conjunto Crit $(f)$ é finito.

Lema 3.2.9. Seja $p \in$ Crit $_{c}$. Se $a<c$ é tal que não há valores críticos de $f$ em $[a, c)$, entäo, toda linha de fluxo nâo constante contida em $\mathcal{W}_{u}(p)$ tem interseção com o nível $f^{-1}(a)$, i.e., para cada $x \in \mathcal{W}_{u}(p) \backslash\{p\}$, existe $t \in \mathbb{R} \operatorname{com} f(t \cdot x)=a$.

Demonstração. Seja $x \in \mathcal{W}_{u}(p) \backslash\{p\}$, então $f(x)<f(p)=c$. Se $f(x) \leq a$, como $\lim _{t \rightarrow-\infty} t \cdot x=p$, temos que quando $t \rightarrow-\infty, f(t \cdot x) \rightarrow f(p)=c>a$; daí, existe $t \leq 0$ tal que $f(t \cdot x)=a$. Se $f(x) \geq a$, basta provar que $f(t \cdot x) \leq a$ para algum $t \geq 0$. Se para cada $t \geq 0, f(t \cdot x)>a$, então, segue do corolário (2.4.4) que $\lim _{t \rightarrow+\infty} t \cdot x=y$ é um ponto crítico, com $c>f(y) \geq a$ o que contradiz o fato que $\operatorname{Crit}(f) \cap f^{-1}([a, c))=\emptyset$.

Observação 3.2.10. Se $p \in M \cap$ Crit $_{c} \operatorname{com} n_{-}(p)=k$, dado a $\in \mathbb{R}$ suficientemente próximo de c. Da observação (2.4.6) temos $\mathcal{W}_{u}(p) \cap f^{-1}(a) \simeq S^{k-1}$; se $b \in \mathbb{R}$ é tal que toda linha de fluxo não constante contida em $\mathcal{W}_{u}(p)$ tem interseção com o nível $f^{-1}(b)$, entäo $\mathcal{W}_{u}(p) \cap f^{-1}(a) \simeq \mathcal{W}_{u}(p) \cap f^{-1}(b)$. Com efeito, se $x \in \mathcal{W}_{u}(p) \cap f^{-1}(a)$, existe $t_{0} \in \mathbb{R}$, tal que $f\left(t_{0} \cdot x\right)=b$, pela continuidade do fluxo temos que a aplicação $\pi$ que associa a cada $x \in \mathcal{W}_{u}(p) \cap f^{-1}(a)$ o ponto $t_{0} \cdot x$ é contínua. Igualmente, se $t_{0} \cdot x=x_{1}$, da observação curvas temos que $\sigma_{x_{1}}(t)=\sigma_{x}\left(t+t_{0}\right)$, logo evaluando em $-t_{0}$, obtemos o valor $x \in \mathcal{W}_{u}(p) \cap f^{-1}(a)$ a aplicação que a cada ponto em $\mathcal{W}_{u}(p) \cap f^{-1}(b)$ associa o correspondênte valor em $\mathcal{W}_{u}(p) \cap f^{-1}(a)$ também é contínua é inversa de $\pi$. Observamos que um caso particular no que o anterior é certo é quando $f^{-1}([b, a)) \cap \operatorname{Crit}(f)=\emptyset$.

A continuação provamos o resultado esperado.

Proposição 3.2.11. Seja $f: M \rightarrow \mathbb{R}$ uma função de Morse definida numa variedade diferenciável compacta de dimensão $n$. Além disso, assumimos que $f$ satisfaz a condição de Morse-Smale de ordem 1. Então, dados $p, q \in \operatorname{Crit}(f)$, com $n_{-}(p)-n_{-}(q)=1$, existe um numero finito de linhas de fluxo para $-\nabla f$ ligando $p$ com $q$.

Demonstraçäo. Seja $a<f(p)$, tal que não há valores críticos de $f$ em $[a, f(p))$. Segue do lema (3.2.9), que toda linha de fluxo não constante para $-\nabla f$ e contida em $\mathcal{W}_{u}(p)$ tem interseção com o nível $f^{-1}(a)$, como $f$ decresce ao longo das linhas de fuxo, tal interseção é única, i.e., existe uma bijeção entre o conjunto das linhas de fluxo não constante para $-\nabla f$ contidas em $\mathcal{W}_{u}(p)$ e o conjunto $\mathcal{W}_{u}(p) \cap f^{-1}(a)$. Ou seja, existe una uma bijeção entre o

\footnotetext{
${ }^{1}$ A continuidade de $\pi$ empregada aqui é mostrada usando as mesmas idéias que aparecem na prova do lema $(3.4 .7)$
} 
conjunto das linhas de fluxo não constante para $-\nabla f$ ligando $p$ com $q$, e o conjunto $\mathcal{W}_{u}(p) \cap$ $\mathcal{W}_{s}(q) \cap f^{-1}(a)$; provemos que tal conjunto é finito. Com efeito, segue do corolário (3.2.2) que $\mathcal{W}_{u}(p) \cap \mathcal{W}_{s}(q) \cap f^{-1}(a)$ é uma variedade diferenciável de dimensão zero, portanto $\mathcal{W}_{u}(p) \cap \mathcal{W}_{s}(q) \cap f^{-1}(a)$ é um subconjunto discreto de $M$; vejamos que é compacto. Com efeito, segue da obsevação (3.2.10) que $\mathcal{W}_{u}(p) \cap f^{-1}(a) \simeq S^{n_{-}(p)-1}$, portanto é compacto. Seja $x$ um ponto no fecho de $\mathcal{W}_{u}(p) \cap \mathcal{W}_{s}(q) \cap f^{-1}(a) ;$ daí $x \in \mathcal{W}_{u}(p) \cap f^{-1}(a)$ e $x$ está no fecho de $\mathcal{W}_{s}(q)$. O lema (3.2.8) garante a existência de uma $k$-step linha de fluxo de $q_{1}=\lim _{t \rightarrow+\infty} t \cdot x$ para $q$, portanto teríamos uma $(k+1)$-step linha de fluxo de $p$ para $q$, daí $1=n_{-}(p)-n_{-}(q) \geq k+1, \operatorname{logo} k=0$ e $q=q_{1}$. Logo $x \in \mathcal{W}_{s}(q) \cap f^{-1}(a)$.

O importante do resultado acima é que permite a definição na próxima seção do complexo de Morse-Witten. Finalizamos esta seção com o seguinte resultado sobre deformação dos níveis de uma função de Morse.

Observação 3.2.12. Na prova da proposição (3.2.11) se $f^{-1}((f(q), a]) \cap \operatorname{Crit}(f)=\emptyset$, entâo é claro do lema (2.4.5) e da observação (3.2.10) que $\mathcal{W}_{s}(q) \cap f^{-1}(a)$ é homeomorfo à esfera de dimensão $\operatorname{dim} M-n_{-}(q)-1$.

Lema 3.2.13. Sejam $a, b \in \mathbb{R}$ tais que $a<b$. Se $f: M \rightarrow \mathbb{R}$ é uma aplicação diferenciável

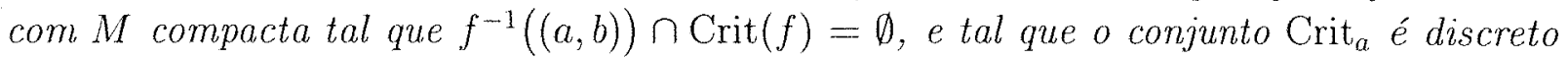
no conjunto Crit $(f)$. (Em particular se $f$ é de Morse). Então, $f^{a}$ é um retrato por deformação de $f^{b} \backslash \mathrm{Crit}_{b}$.

Demonstração. Dado $x \in f^{b} \backslash$ Crit $_{b}, \operatorname{com} f(x)>a$, é claro que $x \in \mathcal{D}_{a}$ ou $x \in \mathcal{M}_{a}$. Com

$$
\begin{aligned}
& \mathcal{D}_{a}=\left\{x \in f^{-1}((a, b)): \exists t>0 \text { e } f(t \cdot x)=a\right\} \\
& \mathcal{M}_{a}=\left\{x \in f^{-1}((a, b)): \lim _{t \rightarrow+\infty} t \cdot x=p \in \text { Crit }_{a}\right\}
\end{aligned}
$$

Se $x \in \mathcal{D}_{a}, t(x)$ denota o número real $t$ tal que $f(t \cdot x)=a$. Definimos a seguinte função:

$$
\rho(x, t)= \begin{cases}t \cdot x & \text { se } x \in \mathcal{D}_{a} \text { e } 0 \leq t \leq t(x) \\ t(x) \cdot x & \text { se } x \in \mathcal{D}_{a} \text { e } t(x) \leq t<\infty \\ t \cdot x & \text { se } x \in \mathcal{M}_{a} \\ x & \text { se } x \in f^{a}\end{cases}
$$

Cuja continuidade segue das idéias que aparecem na prova do lema (3.4.7). Definimos a deformação $\mathrm{em} f^{b} \backslash \mathrm{Crit}_{b} \times[0,1]$ como:

$$
\mathcal{F}(x, t)= \begin{cases}\rho\left(x, \frac{t}{1-t}\right) & \text { se } 0 \leq t<1 \\ \lim _{t \rightarrow+\infty} t \cdot x & \text { se } t=1\end{cases}
$$

A continuidade de $\mathcal{F}$ em $t=1$ segue do lema (2.4.1). 


\subsection{O complexo de Morse-Witten}

Nesta seção é considerada uma função de Morse $f: M \rightarrow \mathbb{R}$, a qual satisfaz a condição genérica de Morse Smale de ordem 1, e é definda numa variedade Riemanniana compacta. Nosso objetivo, é utilizando o fluxo do campo $-\nabla f$ associar um complexo de cadeia $\left(\mathcal{C}_{k}(f), \partial_{k}\right)_{k \in \mathbb{Z}}$ a $f$, o qual é chamado $O$ complexo de Morse-Witten para $f$. Dado que $f$ satisfaz a condição de Morse-Smale de ordem 1, vimos na seção anterior que dados pontos críticos distintos $p$ e $q$ de $f, \operatorname{com} n_{-}(p)-n_{-}(q)=1$, e tais que as variedades $\mathcal{W}_{u}(p), \mathcal{W}_{s}(q)$ são não disjuntas, então $\mathcal{S}=\mathcal{W}_{u}(p) \cap \mathcal{W}_{s}(q)$ é uma a variedade com dimensão 1 que consiste de uma coleção finita de linhas de fluxo de $-\nabla f$ ligando $p$ com $q$. Além do mais, $\mathcal{W}_{u}(p)$ é homeomorfa a $\mathbb{R}^{n_{-}(p)}$ (vide observação 2.4 .6 ) portanto, $\mathcal{W}_{u}(p)$ é simplesmente conexa e orientável (vide proposição 3.1.4). Similarmente, $\mathcal{W}_{s}(p)$ é homeomorfa a $\mathbb{R}^{n_{+}(p)}$, donde simplesmente conexa e transversalmente orientável. Para definir o complexo de MorseWitten para $f$ a idéia é a seguinte: Para cada $p \in$ Crit $(f)$, é escolhida uma orientação no espaço vetorial $T_{p}\left(\mathcal{W}_{u}(p)\right)$; tal escolha induz uma orientação em $\mathcal{W}_{u}(p)$ e uma orientação transversa em $\mathcal{W}_{s}(p)$. Daí para cada par de pontos $p, q \in \operatorname{Crit}(f) \operatorname{com} n_{-}(p)-n_{-}(q)=1$ da observação (3.1.5) obtemos uma orientação na interseção $\mathcal{W}_{u}(p) \cap \mathcal{W}_{s}(q)$. Note que tal interseção possue também uma orientação induzida pelo própio campo $-\nabla f$, que gera seu espaço tangente. Diremos que uma linha de fluxo ligando $p$ com $q$ é positiva com sinal +1 , (resp, negativa com sinal -1 ) se a orientação induzida em tal linha de fluxo pela orientação de $\mathcal{W}_{u}(p)$ e pela orientação transversa de $\mathcal{W}_{s}(p)$ coincidem (resp, não coincidem) com a orientação induzida por $-\nabla f$.

Definamos pois a cadeia; para $k \geq 0$, é definido $\mathcal{C}_{k}(f)$ como o grupo abeliano livre gerado pelo conjunto dos pontos críticos de $f$ com índice $k$; se $k<0$, definimos $\mathcal{C}_{k}(f)=0$. Dados $p, q \in \operatorname{Crit}(f), \operatorname{com} n_{-}(p)=k$ e $n_{-}(q)=k-1$, é preciso definir o coeficiente para $q$ na expressão para o bordo de $p, \partial_{k}(p)$; mas tal coeficiente é definido como a soma algébrica dos sinais das linhas de fluxo de $-\nabla f$ ligando $p \operatorname{com} q$. Note que tal definição tem sentido pelo fato que a soma é feita num conjunto finito. Assim, é definido o número de interseção de $p$ e q:

$$
n(p, q)=\sum_{\operatorname{Im} \sigma \subset \mathcal{S}} \operatorname{sinal}(\sigma)
$$

em que $\mathcal{S}=\mathcal{W}_{u}(p) \cap \mathcal{W}_{s}(q)$. O operador bordo $\partial_{k}: \mathcal{C}_{k}(f) \rightarrow \mathcal{C}_{k-1}(f)$ é definido por:

$$
\partial_{k}(p)=\sum_{q \in \mathcal{C}_{k-1}(f)} n(p, q) q .
$$

Obtém-se a seguinte seqüência de grupos abelianos e homomorfismos:

$$
\ldots \stackrel{\partial_{k+2}}{\longrightarrow} \mathcal{C}_{k+1}(f) \stackrel{\partial_{k+1}}{\longrightarrow} \mathcal{C}_{k}(f) \stackrel{\partial_{k}}{\longrightarrow} \mathcal{C}_{k-1}(f) \stackrel{\partial_{k-1}}{\longrightarrow} \ldots
$$

Adiante provaremos que $\left(\mathcal{C}_{k}(f), \partial_{k}\right)_{k \in \mathbb{Z}}$ é um complexo de cadeia, i.e., $\partial_{k} \circ \partial_{k+1}=0$ para cada $k \in \mathbb{Z}$. Tal complexo será chamado o complexo de Morse-Witten associado 
a $f$. No restante deste texto faremos um abuso de linguagem pois nos reportaremos a $\left(\mathcal{C}_{k}(f), \partial_{k}\right)_{k \in \mathbb{Z}}$ como o complexo de Morse-Witten, sem ter provado ainda que efetivamente é um complexo de cadeia. A homologia do complexo de Morse-Witten será chamada a homologia de Morse da função $f$.

Observamos o seguinte: se $p$ e $q$ são pontos críticos da $f$ com índices de Morse $k$ e $k-1$ respectivamente, dado um número real a tal que $a<f(p)$ e não há mais valores críticos entre a e $f(p)$, nem entre $a$ e $f(q)$, considerando inclusão $i: \mathcal{W}_{u}(p) \cap f^{-1}(a) \rightarrow$ $f^{-1}(a)$, observamos que a codimensão de $\mathcal{W}_{s}(q) \cap f^{-1}(a)$ em $f^{-1}(a)$ é igual à dimensão de $\mathcal{W}_{u}(p) \cap f^{-1}(a)$ que é $k-1$. Portanto, da definição (3.1.11) temos que o número de interseção das esferas $\mathcal{W}_{u}(p) \cap f^{-1}(a)$ e $\mathcal{W}_{s}(q) \cap f^{-1}(a)$ em $f^{-1}(a)$ é dado por:

$$
\sum_{x \in \mathcal{S} \cap f^{-1}(a)} \sin a l(x)
$$

em que $\operatorname{sinal}(x)=+1$, se o isomorfismo:

$$
T_{x}\left(\mathcal{W}_{u}(p) \cap f^{-1}(a)\right) \stackrel{\pi \mathrm{d} i(x)}{\longrightarrow} \frac{T_{x}\left(f^{-1}(a)\right)}{T_{x}\left(\mathcal{W}_{s}(q) \cap f^{-1}(a)\right)}
$$

é positivo; caso contrário, $\sin a l(x)=-1$. A pergunta agora é qual é a relação entre as somas (3.6) e (3.9)?; para dar resposta a tal pergunta, inicialmente observamos que a aplicação $T_{x}\left(f^{-1}(a)\right) \stackrel{\pi}{\longrightarrow} T_{x} M / T_{x}\left(\mathcal{W}_{s}(q)\right)$ é sobrejectiva e $\operatorname{Ker}(\pi)=T_{x}\left(f^{-1}(a) \cap \mathcal{W}_{s}(q)\right)$, portanto temos um isomorfismo:

$$
\frac{T_{x}\left(f^{-1}(a)\right)}{T_{x}\left(f^{-1}(a) \cap \mathcal{W}_{s}(q)\right)} \stackrel{T_{x} M}{T_{x}\left(\mathcal{W}_{s}(q)\right)} .
$$

Como $T_{x} M / T_{x}\left(\mathcal{W}_{s}(q)\right)$ é orientado, segue do isomorfismo (3.10) que posso determinar quando uma base para $T_{x}\left(f^{-1}(a)\right) / T_{x}\left(\mathcal{W}_{s}(q)\right)$ é positiva ou negativa, i.e., posso orientar tal espaço. Seja $\left(b_{1}, \ldots, b_{k-1}\right)$ base para $T_{x}\left(\mathcal{W}_{u}(p) \cap f^{-1}(a)\right)$ tal que $\left(-\nabla f(x), b_{1}, \ldots, b_{k-1}\right)$ seja base positiva de $T_{x}\left(\mathcal{W}_{u}(p)\right)$. Logo a linha de fluxo $\sigma$ passando pelo ponto $x \in$ $\mathcal{W}_{u}(p) \cap \mathcal{W}_{s}(q)$, tem sinal positivo se, e somente se, $\left[b_{1}\right], \ldots,\left[b_{k-1}\right]$ é base positiva de $T_{x} M / T_{x}\left(\mathcal{W}_{s}(q)\right)$. Por outro lado, se $\left(b_{1}, \ldots, b_{k-1}\right)$ base positiva para $T_{x}\left(\mathcal{W}_{u}(p) \cap f^{-1}(a)\right)$, então $u m$ ponto $x \in \mathcal{W}_{u}(p) \cap \mathcal{W}_{s}(q)$ tem sinal positivo (segundo a definição (3.1.11)) se, e somente se, $\left[b_{1}\right], \ldots,\left[b_{k-1}\right]$ é base positiva de $T_{x}\left(f^{-1}(a)\right) / T_{x}\left(\mathcal{W}_{s}(q) \cap f^{-1}(a)\right)$ se, e somente se, $\left[b_{1}\right], \ldots,\left[b_{k-1}\right]$ é base positiva de $T_{x} M / T_{x}\left(\mathcal{W}_{s}(q)\right)$. Portanto temos que o número de interseção entre os pontos $p$ e $q, n(p, q)$, e só o número de interseção das esferas $\mathcal{W}_{s}(q) \cap f^{-1}(a)$ e $\mathcal{W}_{u}(p) \cap f^{-1}(a)$ no ambiente $f^{-1}(a)$.

\subsection{Teorema do Reordenamento}

Nesta seção, nosso objetivo é obter um resultado o qual nos permite provar o resultado central deste trabalho para o caso particular das funções definidas a seguir. 
Definição 3.4.1. Uma aplicação diferenciável $f: M \rightarrow \mathbb{R}$ é dita auto-indexante o função de Smale, se para cada ponto crítico $p$, temos $f(p)=n_{-}(p)$.

Observe que se $f: M \rightarrow \mathbb{R}$ é auto-indexante, e $p, q \in \operatorname{Crit}(f) \operatorname{com} n_{-}(p) \leq n_{-}(q)$, dado $x \in \mathcal{W}_{u}(p) \cap \mathcal{W}_{s}(q)$ teríamos $f(x)<f(p)=n_{-}(p) \leq n_{-}(q)=f(q)<f(x)$, portanto $\mathcal{W}_{u}(p) \cap \mathcal{W}_{s}(q)=\emptyset$, daí, $f$ satisfaz a condição de Morse-Smale de ordem $k=0$.

O seguinte resultado é adaptado de idéias encontradas em [7].

Teorema 3.4.2. Sejam $(M, g)$ uma variedade Riemanniana compacta e $f: M \rightarrow \mathbb{R}$ uma função de Morse que satisfaz a condição de Morse-Smale de ordem $k=0$. Então, existem uma função diferenciável $\tilde{f}: M \rightarrow \mathbb{R}$ e uma métrica Riemanniana $\tilde{g}$ em $M$ de modo que:

- O gradiente de $f$ com respeito à métrica $g$ é igual ao gradiente de $\tilde{f}$ com respeito à métrica $\tilde{g}$;

- $\tilde{f}$ é auto-indexante.

Observação 3.4.3. Se $\tilde{f}$ e $\tilde{g}$, são como na proposiçấo acima, então o complexo de MorseWitten associado a $f$ e $g$ coincide com o complexo de Morse-Witten associado a $\tilde{f}$ e $\tilde{g}$, já que a definição do complexo de Morse-Witten depende apenas do campo gradiente. Isto sem perda de generalidade, nos permitirá reduzir a prova do teorema 5.1.1 para o caso de funções auto-indexantes.

A demonstração do teorema 3.4 .2 acima seguirá dos lemas a seguir.

Lema 3.4.4. Seja $M$ uma variedade diferenciável, $\xi \in \mathfrak{X}(M)$ e $f: M \rightarrow \mathbb{R}$ uma aplicação diferenciável. Se para cada $x \in M$, existem um aberto $U_{x} \subset M$ contendo $x$, e uma métrica Riemanniana g em $U_{x}$ tal que $\left.\xi\right|_{U_{x}}=\left.\nabla f\right|_{U_{x}}$ respeito a g, então existe uma métrica Riemanniana $\tilde{g}$ em $M$ tal que $\xi=\nabla f$ respeito a $\tilde{g}$.

Demonstração. Denotamos por $g_{x}$ a métrica Riemanniana em $U_{x}$, tal que $\left.\xi\right|_{U_{x}}=\left.\nabla f\right|_{U_{x}}$ com respeito a $g_{x}$. Seja $\left(\zeta_{x}\right)_{x \in M}$ uma partição da unidade subordinada à cobertura $\left(U_{x}\right)_{x \in M} ; \tilde{g}_{x}=g_{x} \zeta_{x},\left(\operatorname{Supp} \tilde{g}_{x}\right)_{x \in M}=\left(\operatorname{Supp} \zeta_{x}\right)_{x \in M} \subset U_{x}$ é localmente finita, então, a soma $\tilde{g}=\sum_{x \in M} \tilde{g}_{x}$ é uma função bem definida e diferenciável. A função $\tilde{g}$ assim definida é a métrica desejada.

Lema 3.4.5. Seja $f: M \rightarrow \mathbb{R}$ uma função diferenciável sem pontos críticos. Seja $\xi \in$ $\mathfrak{X}^{k}(M)$, tal que $\xi(f)>0$. Então existe uma métrica Riemanniana $g$ em $M$, tal que $\xi$ éo gradiente de $f$ com respeito à $g$.

Demonstraçâo. A prova deste lema segue do seguinte resultado de algebra linear. Se $Y$ é um espaço vetorial real com produto interno e dimensão finita e $\alpha \in V^{*}$. Dado $v \in V$ tal que $v \in(\operatorname{Ker}(\alpha))^{\perp}$ e $\alpha(v)=\|v\|^{2}$, então $\alpha(\cdot)=\langle v, \cdot\rangle$. Dado $x \in M$, existe uma carta 
$\varphi: U \rightarrow \widetilde{U}$ em $M$ tal que $f \circ \varphi^{-1}\left(x_{1}, \ldots, x_{n}\right)=x_{1}$ para cada $\left(x_{1}, \ldots, x_{n}\right) \in \widetilde{U}$. Seja $\left\{\partial_{i}(x)\right\}_{i=1}^{n}$ base para $T_{x} M$ associada a tal carta. $\left\{\partial_{i}(x)\right\}_{i=2}^{n}$ é base para o $\operatorname{Ker}(\mathrm{d} f(x))$, seja $v=\frac{\xi(f)(x)}{\|\xi(x)\|^{2}} \xi(x)$ defino a métrica $g$ como sendo $g(x)$ o produto interno em $T_{x} M$ tal que a base $\left(v, \partial_{i}(x)\right)_{i=2}^{n}$ seja ortonormal.

Corolário 3.4.6. Sejam $f: M \rightarrow \mathbb{R}$ uma função diferenciável e $\xi \in \mathfrak{X}^{k}(M)$. Suponha que $\xi(f)>0 \mathrm{em} M \backslash \operatorname{Crit}(f)$ e que para cada $x \in \operatorname{Crit}(f)$ existe um aberto $U_{x} \subset M$ contendo $x$, e uma métrica Riemanniana $g(x)$ em $U_{x}$ tal que $\left.\xi\right|_{U_{x}}=\left.\nabla f\right|_{U_{x}}$ com respeito a $g(x)$. Então existe uma métrica Riemanniana $\tilde{g}$ em $M$, tal que $\xi$ é o gradiente de $f$ com respeito à $\tilde{g}$.

Lema 3.4.7. Seja $f:(M, g) \rightarrow \mathbb{R}$ uma funçấo de Morse com $M$ variedade Riemanniana compacta. Sejam $a, b \in \mathbb{R}$ níveis regulares de $f$, tais que $f^{-1}([a, b]) \cap \operatorname{Crit}(f)=\left\{p_{1}, \ldots p_{k}\right\}$. Assumimos que para cada $i, j=1, \ldots, k$ não há linhas de fluxo para $-\nabla f$ ligando $p_{i}$ com $p_{j}$, nem $p_{j}$ com $p_{i}$. Sejam $\left(c_{i}\right)_{i=1}^{k}$ em $] a, b[$. Então, existe uma função diferenciável $\tilde{f}: M \rightarrow \mathbb{R}$ e uma métrica Riemanniana $\tilde{g}$ em $M$ tal que;

- O gradiente de $f$ com respeito à métrica $g$ é igual ao gradiente de $\tilde{f}$ com respeito à métrica $\tilde{g}$;

- $f=\tilde{f}$ fora de $f^{-1}(] a, b[)$;

- $\tilde{f}\left(p_{i}\right)=c_{i}$.

Demonstração. Denotamos por $K_{p_{i}}$ o conjunto $\left(\mathcal{W}_{u}\left(p_{i}\right) \cup \mathcal{W}_{s}\left(p_{i}\right)\right) \cap f^{-1}([a, b])$, e seja $K=\cup_{i=1}^{k} K_{p_{i}}$. É claro pela hipótese que $K_{p_{i}} \cap K_{p_{j}}=\emptyset$ para $i \neq j$, além do mais, para cada $x$ fora de $K$ e em $f^{-1}([a, b])$, as trajetórias das linhas de fliuxo de $-\nabla f$ passando por $x$ têm interseção com os niveis $a$ e $b$. Consideremos o conjunto:

$$
\mathcal{D}_{a}=\{x \in M \backslash \operatorname{Crit}(f): f(t \cdot x)=a \text { para algum } t \in \mathbb{R}\} .
$$

Definimos a aplicação $\pi: \mathcal{D}_{a} \rightarrow f^{-1}(a)$ como: $\pi(x)=t \cdot x$, de modo que $f(t \cdot x)=a$. Observamos que se $x \in \mathcal{D}_{a}, \pi$ é constante ao longo da linha de fluxo para $-\nabla f$ passando por $x$. Vamos mostrar que $\mathcal{D}_{a}$ é aberto e $\pi$ é diferenciável. Com efeito, vejamos que dado $x \in \mathcal{D}_{a}$, existe uma vizinhança aberta $V$ de $x$ tal que $V \subset \mathcal{D}_{a}$ e $\left.\pi\right|_{V}$ é diferenciável. Consideremos a função $\gamma: \mathcal{D}_{a} \times \mathbb{R} \rightarrow \mathbb{R}$ definida como: $\mathcal{D}_{a} \times \mathbb{R} \ni(x, t) \mapsto f(t \cdot x) \in \mathbb{R}$. Dado $\left(x_{0}, t_{0}\right) \in \mathcal{D}_{a} \times \mathbb{R}, \operatorname{com} f\left(t_{0} \cdot x_{0}\right)=a$, como $\frac{\partial \gamma}{\partial t}$ não é zero, segue do teorema da função implicita que existem abertos $V$ e $W$ tais que $x_{0} \in V \subset \mathcal{D}_{a}$ e $t_{0} \in W \subset \mathbb{R}$ e uma função diferenciável $w: V \rightarrow W$, com $w\left(x_{0}\right)=t_{0}$ tal que $\gamma(x, w(x))=f(w(x) \cdot x)=a$ para cada $x \in V, \log$ a função $h: V \rightarrow \mathbb{R} \times \mathcal{D}_{a}$ definida por $h(x)=(w(x), x)$ é diferenciável $\mathrm{e}$ portanto compondo $h$ com o fluxo $F$ de $-\nabla f$, obtemos $\left.\pi\right|_{V}=F \circ h$ diferenciável.

Os conjuntos $f^{-1}(a) \cap \mathcal{W}_{u}\left(p_{i}\right)$ são compactos (vide observação 2.4.6) disjuntos; pelo lema de Urysohn diferenciável existe uma função diferenciável $\mu: f^{-1}(a) \rightarrow \mathbb{R}$ tal que 
$\left.\mu\right|_{\overline{V_{i}}}=i-1$, em que os $V_{i}$ são abertos em $f^{-1}(a)$ com fecho disjunto e $\mathcal{W}_{u}\left(p_{i}\right) \cap f^{-1}(a) \subset V_{i}$. Como $V_{i}$ é uma vizinhança aberta em $f^{-1}(a)$ de $\mathcal{W}_{u}\left(p_{i}\right) \cap f^{-1}(a), \pi^{-1}\left(V_{i}\right)$ é um aberto que contém $\mathcal{W}_{u}\left(p_{i}\right) \backslash\left\{p_{i}\right\}$; segue do corolário 2.2 .23 que existe um aberto $U_{i}$ em $M$ contendo $\mathcal{W}_{s}\left(p_{i}\right)$, tal que para cada $x \in U_{i}, x \in \mathcal{W}_{s}\left(p_{i}\right)$ ou $t \cdot x \in \pi^{-1}\left(V_{i}\right)$ para algum $t>0$. Logo, para $x \in U_{i} \backslash \mathcal{W}_{s}\left(p_{i}\right)$, temos que $\pi(x) \in V_{i}$, i.e., $U_{i}$ é uma vizinhança de $\mathcal{W}_{s}\left(p_{i}\right)$ tal que $\pi\left(U_{i} \cap \mathcal{D}_{a}\right) \subset V_{i}$. Definimos $\bar{\mu}: f^{-1}([a, b]) \rightarrow \mathbb{R}$ como:

$$
\bar{\mu}= \begin{cases}\mu \circ \pi & \text { fora de } K \\ i-1 & \text { em } K_{p_{i}} .\end{cases}
$$

O fato que $\bar{\mu}$ é diferenciável segue da diferenciabilidade de $\mu$ e $\pi$ e do fato que $\bar{\mu}$ é constante e igual a $i-1$ no aberto $U_{i}$ contendo $\mathcal{W}_{s}\left(p_{i}\right)$.

O segundo ingrediente para obter a função procurada $\tilde{f}$, é uma aplicação diferenciável $\mathbb{R}^{2} \ni(x, y) \mapsto G(x, y) \in \mathbb{R}$ em duas variáveis satisfazendo as seguintes condições:

- Para cada par $(x, y) \in \mathbb{R}^{2}, \frac{\partial G}{\partial x}(x, y)>0$.

- Para cada $y \in \mathbb{R}$ fixo, $G(a, y)=a$ e $G(b, y)=b$.

- $G\left(f\left(p_{i}\right), i-1\right)=c_{i}$, para cada $i=1, \ldots, k$.

- $\frac{\partial G}{\partial x}(x, i-1)=1$ para todo $x$ em alguma vizinhança de $f\left(p_{i}\right), i=1, \ldots, k$.

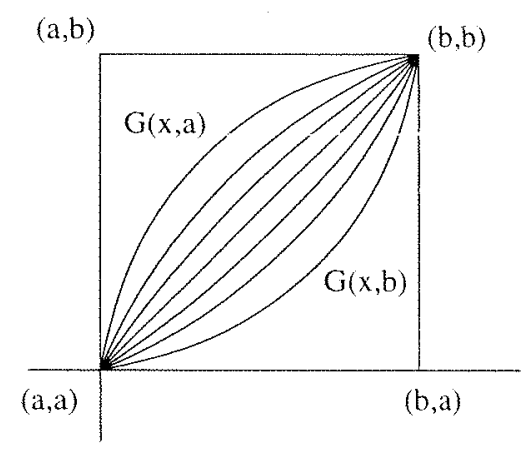

Figura 3.1: Projeção do gráfico de $G$ no plano $x z$.

Definimos a função $\tilde{f}$ como segue:

$$
\tilde{f}(x)= \begin{cases}G(f(x), \bar{\mu}(x)) & x \in f^{-1}([a, b]) \\ f(x) & x \text { fora de } f^{-1}([a, b]) .\end{cases}
$$

É fácil ver que a função $\tilde{f}$ satisfaz as condições desejadas; a métrica $\tilde{g}$ é obtida usando o corolário 3.4.6. 


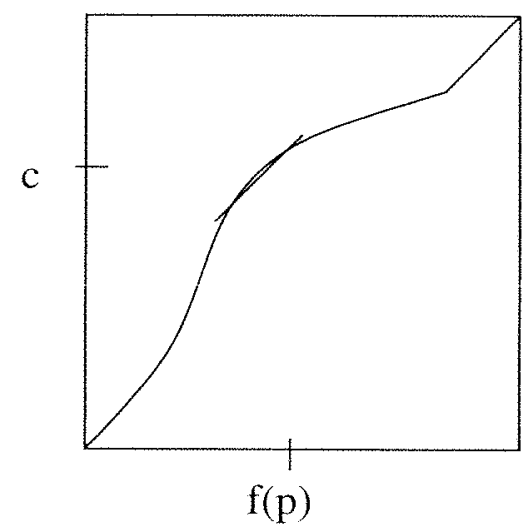

Figura 3.2: Gráfico de $x \mapsto G(x, i-1)$.

Demonstração do teorema 3.4.2. Como $f$ satisfaz a condição de Morse-Smale de ordem zero então, dados pontos críticos $p$ e $q \operatorname{com} f(p) \geq f(q)$ e $n_{-}(p) \leq n_{-}(q)$ então não existem linhas de fluxo de $-\nabla f$ ligando $p$ a $q$; de fato, a existência de uma linha de fluxo saindo de $p$ e chegando em $q$ implicaria $n_{-}(q)<n_{-}(p)$ e a existência de uma linha de fluxo saindo de $q$ e chegando em $p$ implicaria $f(q)<f(p)$. Uma aplicação sucessiva do lema 3.4 .7 nos fornece uma função $\tilde{f}: M \rightarrow \mathbb{R}$ e uma métrica Riemanniana $\tilde{g}$ em $M$ tais que o gradiente de $\tilde{f}$ com respeito a $\tilde{g}$ é igual ao gradiente de $f$ com respeito a $g$ e tal que:

$$
n_{-}(p)<n_{-}(q) \Longleftrightarrow \tilde{f}(p)<\tilde{f}(q) \text { e } n_{-}(p)=n_{-}(q) \Longleftrightarrow \tilde{f}(p)=\tilde{f}(q),
$$

para quaisquer $p, q \in \operatorname{Crit}(f)=\operatorname{Crit}(\tilde{f})$. A conclusão final é obtida agora compondo $\tilde{f}$ à esquerda com um difeomorfismo crescente apropriado $\alpha: \mathbb{R} \rightarrow \mathbb{R}$ e multiplicando a métrica $\tilde{g}$ por $\alpha^{\prime} \circ f$. 


\section{Capítulo 4}

\section{Tópicos em Topologia Algébrica}

Neste capítulo provaremos alguns resultados da topologia algébrica úteis na prova do resultado principal deste trabalho.

\subsection{Homologia dos Espaços Filtrados}

Associamos um complexo de cadeia a um espaço filtrado. Com algumas hipóteses sobre a filtração, provamos que a homologia de tal complexo é naturalmente isomorfa à homologia singular do espaço. Empregamos como referencia básica para notação, terminologia e resultados não provados [9].

Se $A$ é um subespaço de um espaço topológico $X$, dizemos que $(X, A)$ é um par de espaços topológicos, para cada $p$, o grupo das $p$ cadeias singulares relativas do par $(X, A)$ é denotado por $\mathcal{S}_{p}(X, A)=\mathcal{S}_{p}(X) / \mathcal{S}_{p}(A)$, obtém-se assim um complexo de cadeia $(\mathcal{S}(X, A), \partial)$, em que o operador bordo é o induzido no quociente pelo operador bordo do complexo de cadeia singular associado a $X$; os grupos de homologia deste complexo são chamados grupos de homologia singular do $\operatorname{par}(X, A)$, são denotados por $H_{p}(X, A)$, e são identificados com o quociente $Z_{p}(X, A) / B_{p}(X, A)$, em que:

$$
\begin{aligned}
& Z_{p}(X, A)=\left\{c \in \mathcal{S}_{p}(X): \partial_{p}(c) \in \mathcal{S}_{p-1}(A)\right\}=\partial_{p}^{-1}\left(\mathcal{S}_{p-1}(A)\right) \\
& B_{p}(X, A)=\left\{\partial_{p+1} c+d: c \in \mathcal{S}_{p+1}(X), d \in \mathcal{S}_{p}(A)\right\}=B_{p}(X)+\mathcal{S}_{p}(A) .
\end{aligned}
$$

Por uma aplicação de pares entendemos uma função contínua $f:(X, A) \rightarrow(Y, B)$ tal que $f(A) \subset B$; denotamos por $f_{*}: H_{p}(X, A) \rightarrow H_{p}(Y, B)$ o homomorfismo em homologia induzido por $f$. Os seguintes resultados são empregados no restante do texto.

(a) Se $i d: X \rightarrow X$ é a aplicação identidade, e $f: X \rightarrow Y, g: Y \rightarrow Z$ são contínuas, então $(i d)_{*}=i d$, e $(g \circ f)_{*}=g_{*} \circ f_{*}$. 
(b) (Exatidão) Se $B \subset A \subset X$, e $i:(A, B) \rightarrow(X, B), j:(X, B) \rightarrow(X, A)$ são inclusões, então a seqüência em homologia:

$$
\cdots \longrightarrow H_{p+1}(X, A) \stackrel{\partial_{*}}{\longrightarrow} H_{p}(A, B) \stackrel{i_{*}}{\longrightarrow} H_{p}(X, B) \stackrel{j_{*}}{\longrightarrow} H_{p}(X, A) \stackrel{\longrightarrow}{\longrightarrow}
$$

é exata. E é chamada a seqüência exata longa da tripla $(X, A, B)$, além do mais, se $z \in Z_{p}(X, A), \partial_{*}\left(z+B_{p}(X, A)\right)=\partial z+B_{p-1}(A, B)$.

(c) Se $B \subset A \subset X$ e $C \subset D \subset Y$, e $f:(X, A) \rightarrow(Y, D)$ é uma aplicação de pares com $f(B) \subset C$, o seguinte diagrama é conmutativo:

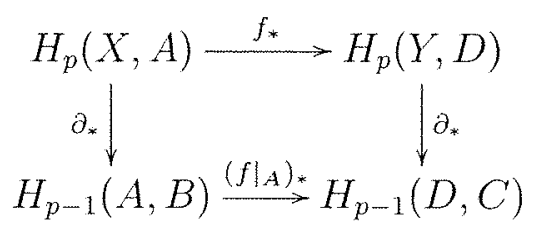

em que $\partial_{*}$ denota o homomorfismo que aparece na seqüencia exata longa da tripla.

(d) (Suporte Compacto) Dado $\alpha \in H_{p}(X, A)$, existe um par compacto $\left(X_{0}, A_{0}\right) \subset(X, A)$ tal que $\alpha$ esta na imagem do homomorfismo $i_{*}: H_{p}\left(X_{0}, A_{0}\right) \rightarrow H_{p}(X, A)$ induzido pela inclusão. Além do mais, se $i:\left(X_{0}, A_{0}\right) \rightarrow(X, A)$ denota a inclusão em que $\left(X_{0}, A_{0}\right)$ é um par compacto, e $\alpha \in H_{p}\left(X_{0}, A_{0}\right)$ com $i_{*}(\alpha)=0$, então existe um par compacto $\left(X_{1}, A_{1}\right)$ e inclusões $\left(X_{0}, A_{0}\right) \stackrel{j}{\longrightarrow}\left(X_{1}, A_{1}\right) \stackrel{k}{\longrightarrow}(X, A)$ tal que $j_{*}(\alpha)=0$.

(e) (Invariança por Homotopia) Se $f$ e $g$ são funções homotópicas, então $f_{*}=g_{*}$.

(f) Se $f:(X, A) \rightarrow(Y, B)$ é uma equivalêcia homotópica, quer dizer se existe uma aplicação $g:(Y, B) \rightarrow(X, A)$ tal que $g \circ f$ e $f \circ g$ são homotópicas às respectivas identidades, então $f_{*}$ é um isomorfismo em homologia. Mais geral se $f: X \rightarrow Y$ e $\left.f\right|_{A}: A \rightarrow B$ são equivalêcias homotópicas, então $f_{*}$ é um isomorfismo. Em particular, se $A \subset X$ é um retrato por deformação de $X$, então para cada $p \in \mathbb{Z}$, $H_{p}(X) \simeq H_{p}(A)$.

(g) (Excisão) Seja $A \subset X$. Se $U \subset X$, tal que $\bar{U}$ esta contido no interior de $A$, então a inclusão $i:(X \backslash U, A \backslash U) \rightarrow(X, A)$ induz um isomorfimo em homologia.

(h) (Descomposição) Se $X=\bigcup_{i \in I} X_{i}$ em que os $X_{i}$ são abertos e disjuntos, $A \subset X$ e $A_{i}=A \cap X_{i}$ então $H_{p}(X, A) \simeq \bigoplus_{i \in I} H_{p}\left(X_{i}, A_{i}\right)$

Observação 4.1.1. Suponha que X é T1, i.e., os pontos de X são fechados. Seja $A \subset X$. Se $A$ contém uma vizinhança do ponto $x \in X$, então segue da propriedade de excisão que $H_{p}(X, X \backslash\{x\}) \simeq H_{p}(A, A \backslash\{x\})$. 
Observação 4.1.2. Suponha que $X$ é Hausdorff e que $p_{1}, \ldots, p_{n} \in X$ são pontos distintos. Afirmamos que para todo $k$ a aplicaçäo:

$$
H_{k}\left(X, X \backslash\left\{p_{1}, \ldots, p_{n}\right\}\right) \longrightarrow \bigoplus_{i=1}^{n} H_{k}\left(X, X \backslash\left\{p_{i}\right\}\right)
$$

induzida por inclusão é um isomorfismo. De fato, isso segue do diagrama:

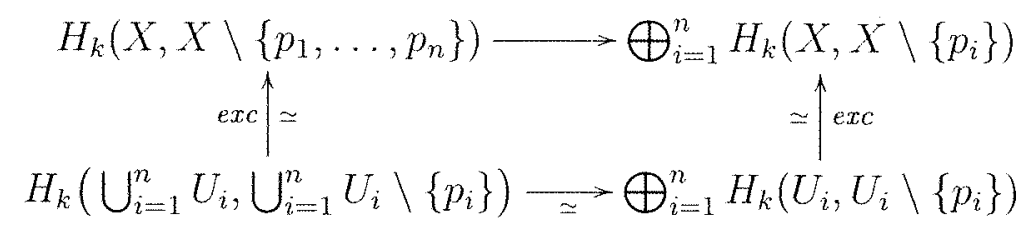

onde $U_{1}, \ldots, U_{n}$ são abertos dois a dois disjuntos em $X$ com $p_{i} \in U_{i}, i=1, \ldots, n$.

Definição 4.1.3. Se $X$ é um espaço topológico, uma filtração de $X$ é uma seqüência de subespaços $\left(X^{p}\right)_{p \in \mathbb{N}}$ tal que para cada $p=1,2 \ldots$ temos $X^{p-1} \subset X^{p}$. Um espaço topológico junto a uma filtração é chamado um espaço filtrado.

Vamos associar um complexo de cadeia à filtração $\left(X^{p}\right)_{p \in \mathbb{N}}$ de $X$. Por simplicidade, escrevemos $X^{p}=\emptyset$ para $p<0 ;(\mathcal{S}(X), \partial)$ denota o complexo de cadeia singular de $X$. Para $p \in \mathbb{Z}$, sejam:

$$
\begin{aligned}
& \mathcal{Z}_{p}(X)=Z_{p}\left(X^{p}, X^{p-1}\right), \\
& \mathcal{B}_{p}(X)=B_{p}\left(X^{p}, X^{p-1}\right) .
\end{aligned}
$$

Como é sabido, $H_{p}\left(X^{p}, X^{p-1}\right) \simeq \mathcal{Z}_{p}(X) / \mathcal{B}_{p}(X)$. $\mathcal{Z}(X)$ e $\mathcal{B}(X)$ são subcomplexos de $\mathcal{S}(X)$, tais que o grupo dos $p$-ciclos em $\mathcal{Z}(X)$ é $Z_{p}\left(X^{p}\right)$ e o grupo dos p-bordos em $\mathcal{Z}(X)$ é $B_{p}\left(X^{p+1}\right) \cap Z_{p}\left(X^{p}\right)$. Para cada $p \in \mathbb{Z}$ definimos:

$$
\mathcal{D}_{p}(X)=\mathcal{Z}_{p}(X) / \mathcal{B}_{p}(X)
$$

então, $(\mathcal{D}(X), \partial)$ é um complexo de cadeia com operador bordo $\partial$ induzido no quociente pelo bordo de $\mathcal{Z}(X)$; o qual coincide com o operador bordo $\partial_{*}$ na sequência exata longa da tripla $\left(X^{p}, X^{p-1}, X^{p-2}\right)$ :

$$
\mathcal{D}_{p}(X)=H_{p}\left(X^{p}, X^{p-1}\right) \stackrel{\partial_{*}}{\longrightarrow} H_{p-1}\left(X^{p-1}, X^{p-2}\right)=\mathcal{D}_{p-1}(X) .
$$

Observação 4.1.4. Se $\epsilon$ denota a aumentação do complexo $(\mathcal{S}(X), \partial)$, e $X^{0}$ não é vazio, dado que $\mathcal{Z}_{0}(X)=\mathcal{S}_{0}\left(X^{0}\right)$, é possível restrigir $\epsilon$ a $\mathcal{S}_{0}\left(X^{0}\right)$ e obter uma aumentação para $\mathcal{Z}(X)$. Além do mais, como $\mathcal{B}_{0}(X)=B_{0}\left(X^{0}\right)$ e $\left.\epsilon\right|_{B_{0}\left(X^{0}\right)}=0$, $\epsilon$ também define uma aumentação do complexo $\mathcal{D}(X)$.

Definição 4.1.5. Se $X$ e $Y$ são espaços filtrados, dizemos que uma função $f: X \rightarrow Y$ preserva filtração se é contínua e para cada $p, f\left(X^{p}\right) \subset\left(Y^{p}\right)$. 
Se $f: X \rightarrow Y$ preserva filtração, é claro que a aplicação de cadeia $f_{\sharp}$ leva $\mathcal{Z}(X)$ em $\mathcal{Z}(Y)$ e $\mathcal{B}(X)$ em $\mathcal{B}(Y)$, portanto induz uma aplicação de cadeia emtre $\mathcal{D}(X)$ e $\mathcal{D}(Y)$ a qual coincide com o homeomorfismo $f_{*}: H_{p}\left(X^{p}, X^{p-1}\right) \rightarrow H_{p}\left(Y^{p}, Y^{p-1}\right)$ induzido por $f$ em homologia relativa. Tal aplicação preserva aumentação se $X^{0}$ não é vazio.

Uma filtração $\left(X^{p}\right)_{p \in \mathbb{N}}$ para um espaço topológico $X$ é dita uma boa filtração, se são satisfeitas as seguintes condições:

- Para cada $p, p \neq i, H_{i}\left(X^{p}, X^{p-1}\right)=0$

- Para cada conjunto compacto $K \subset X$, existe $p$ tal que $K \subset X^{p}$.

Um espaço topológico junto uma boa filtração é chamado espaço bem filtrado.

Como foi dito no inicio da seção, nosso objetivo é mostrar que a homologia do complexo de cadeia associado à filtração de $X, \mathcal{D}(X)$ é naturalmente isomorfa à homologia singular de $X$. Iniciamos com dois lemas úteis.

Lema 4.1.6. Seja $X$ um espaço bem filtrado. Então, para cada $p$, a inclusão induz um isomorfismo em $H_{p}\left(X^{p+1}\right) \rightarrow H_{p}(X)$.

Demonstração. A seqüência exata longa para o par $\left(X^{i+1}, X^{i}\right)$ mostra que

$$
H_{p+1}\left(X^{i+1}, X^{i}\right) \stackrel{\partial_{*}}{\longrightarrow} H_{p}\left(X^{i}\right) \stackrel{i_{*}}{\longrightarrow} H_{p}\left(X^{i+1}\right) \stackrel{j_{*}}{\longrightarrow} H_{p}\left(X^{i+1}, X^{i}\right)
$$

é exata. Para cada $i \geq p+1$, segue-se da hipóteses que os grupos dos extremos são triviais, logo a inclusão induz um isomorfismo $H_{p}\left(X^{i}\right) \rightarrow H_{p}\left(X^{i+1}\right)$. Portanto, a inclusão induz um isomorfismo $H_{p}\left(X^{i}\right) \rightarrow H_{p}\left(X^{j}\right)$, para $j \geq i \geq p+1$.

O resultado segue da propriedade de suporte compacto, seja $\alpha$ uma classe de homologia em $H_{p}(X)$, existe um compacto $K \subset X$ tal que $\alpha$ está na imagem de $H_{p}(K) \rightarrow H_{p}(X)$; como $K$ é compacto, existe um $j$ tal que $K \subset X^{p+j}$. Logo $\alpha$ está na imagem de $H_{p}\left(X^{p+j}\right) \rightarrow H_{p}(X)$. Como $H_{p}\left(X^{p+1}\right) \rightarrow H_{p}\left(X^{p+j}\right)$ é um isomorfismo, $\alpha$ está na imagem de $H_{p}\left(X^{p+1}\right) \rightarrow H_{p}(X)$. Temos provado que $H_{p}\left(X^{p+1}\right) \rightarrow H_{p}(X)$ é sobrejetivo.

Para a injetividade, seja $\alpha$ uma classe de homologia em $H_{P}\left(X^{p+1}\right)$ cuja imagem pela aplicação $H_{p}\left(X^{p+1}\right) \rightarrow H_{p}(X)$ é nula, existe um compacto $K$, e inclusões $X^{p+1} \stackrel{i}{\rightarrow} K \stackrel{j}{\rightarrow} X$ tal que $i_{*}(\alpha)=0$. Além do mais, $K \subset X^{p+t}$ para algum $t$, portanto o isomorfismo $H_{p}\left(X^{p+1}\right) \rightarrow H_{p}\left(X^{p+t}\right)$ leva $\alpha$ no zero, daí, $\alpha=0$.

Lema 4.1.7. Seja $X$ um espaço bem filtrado. Então, para cada $p$, $i$ com $p>i$ temos $H_{p}\left(X^{i}\right)=0$.

Demonstração. Para $j \leq i$, a seqüencia exata da tripla $\left(X^{i}, X^{j}, X^{j-1}\right)$ mostra que

$$
H_{p}\left(X^{j}, X^{j-1}\right) \stackrel{i_{*}}{\longrightarrow} H_{p}\left(X^{i}, X^{j-1}\right) \stackrel{j_{*}}{\longrightarrow} H_{p}\left(X^{i}, X^{j}\right) \stackrel{\partial_{*}}{\longrightarrow} H_{p-1}\left(X^{j}, X^{j-1}\right)
$$


é exata. Para $j \leq p-2$, os grupos dos extremos são triviais, portanto temos que $H_{p}\left(X^{i}, X^{j-1}\right) \rightarrow H_{p}\left(X^{i}, X^{j}\right)$ é um isomorfismo. Dado que $X^{k}=\emptyset$ para $k<0$, a inclusão também induz um isomorfismo $H_{p}\left(X^{i}\right) \rightarrow H_{p}\left(X^{i}, X^{j}\right)$ em que $j=\min \{i, p-2\}$. Se $i \leq p-2, j=i$ e $H_{p}\left(X^{i}, X^{j}\right)=H_{p}\left(X^{i}, X^{i}\right)=0$; casso contrário, $i=p-1 \mathrm{e}$ $H_{p}\left(X^{i}, X^{j}\right)=H_{p}\left(X^{p-1}, X^{p-2}\right)=0$.

Teorema 4.1.8. Seja $X$ um espaço bem filtrado. Então, a inclusão $\mathcal{Z}(X) \rightarrow \mathcal{S}(X)$ induz um isomorfismo em homologia. Se $X^{0} \neq \emptyset$, o resultado é válido em homologia reduzida. Além do mais, se $f: X \rightarrow Y$ preserva filtração, então o seguinte diagrama

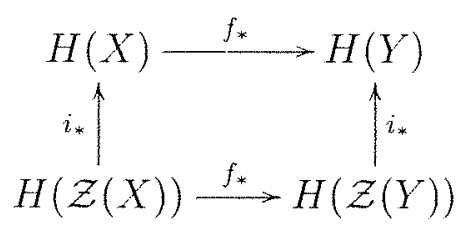

comuta.

Demonstração. Como para cada $p$, o grupo dos p-ciclos em $\mathcal{Z}(X)$ é $Z_{p}\left(X^{p}\right)$, e o grupo dos $p$-bordos é $B_{p}\left(X^{p+1}\right) \cap Z_{p}\left(X^{p}\right)$. Devemos provar que a inclusão induz um isomorfismo:

$$
\frac{Z_{p}\left(X^{p}\right)}{B_{p}\left(X^{p+1}\right) \cap Z_{p}\left(X^{p}\right)} \longrightarrow \frac{Z_{p}(X)}{B_{p}(X)}
$$

Segue do lema (4.1.6), que o homomorfismo:

$$
\frac{Z_{p}\left(X^{p+1}\right)}{B_{p}\left(X^{p+1}\right)} \longrightarrow \frac{Z_{p}(X)}{B_{p}(X)}
$$

induzido pela inclusão é um isomorfismo. A seqüência exata do par $\left(X^{p+1}, X^{p}\right)$ mostra que

$$
H_{p}\left(X^{p}\right) \stackrel{i_{*}}{\longrightarrow} H_{p}\left(X^{p+1}\right) \stackrel{j_{*}}{\longrightarrow} H_{p}\left(X^{p+1}, X^{p}\right)=0
$$

é exata, portanto o homomorfismo:

$$
\frac{Z_{p}\left(X^{p}\right)}{B_{p}\left(X^{p}\right)} \longrightarrow \frac{Z_{p}\left(X^{p+1}\right)}{B_{p}\left(X^{p+1}\right)}
$$

induzido pela inclusão é sobrejetivo; o que implica que $Z_{p}\left(X^{p+1}\right)=Z_{p}\left(X^{p}\right)+B_{p}\left(X^{p+1}\right)$. Logo do teorema de homomorfismos, o homomorfismo:

$$
\frac{Z_{p}\left(X^{p}\right)}{B_{p}\left(X^{p+1}\right) \cap Z_{p}\left(X^{p}\right)} \longrightarrow \frac{Z_{p}\left(X^{p}\right)+B_{p}\left(X^{p+1}\right)}{B_{p}\left(X^{p+1}\right)} \longrightarrow \frac{Z_{p}\left(X^{p+1}\right)}{B_{p}\left(X^{p+1}\right)}
$$

induzido pela inclusão é um isomorfismo. O resultado segue do fato que (4.3) e (4.4) são isomorfismos. Se $X^{0} \neq \emptyset$, a inclusão $\mathcal{Z}(X) \rightarrow \mathcal{D}(X)$ preserva aumentação, e portanto também induz um isomorfismo em homologia reduzida. A naturalidade, segue do fato que (4.2) comuta a nível de cadeia. 
Teorema 4.1.9. Seja $X$ um espaço bem filtrado. Então o quociente $q_{\sharp}: \mathcal{Z}(X) \rightarrow \mathcal{D}(X)$ induz um isomorfismo em homologia. Se $X^{0} \neq \emptyset$, o resultado é válido em homologia reduzida. Além do mais, o isomorfismo é natural i.e., se $f: X \rightarrow Y$ preserva filtração, então o seguinte diagrama:

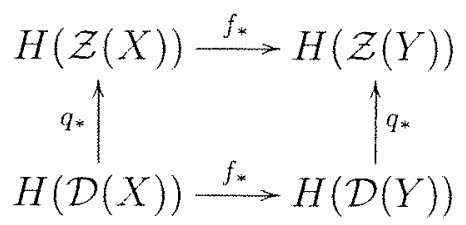

comuta. Em que as flechas horizontais são induzidas por restrições apropiadas de $f_{\sharp}$.

Demonstração. Temos uma seqüência exata curta de complexos de cadeia

$$
0 \longrightarrow \mathcal{B}(X) \stackrel{i_{*}}{\longrightarrow} \mathcal{Z}(X) \stackrel{q_{*}}{\longrightarrow} \mathcal{D}(X) \longrightarrow 0
$$

A correspondente seqüencia exata longa em homologia:

$$
\cdots \longrightarrow H_{p}(\mathcal{B}(X)) \stackrel{i_{*}}{\longrightarrow} H_{p}(\mathcal{Z}(X)) \stackrel{q_{*}}{\longrightarrow} H_{p}(\mathcal{D}(X)) \stackrel{\partial_{*}}{\longrightarrow} H_{p-1}(\mathcal{B}(X)) \longrightarrow \cdots
$$

mostra que o quociente $q_{\sharp}: \mathcal{Z}(X) \rightarrow \mathcal{D}(X)$ induz um isomorfismo em homologia se, e somente se, $\mathcal{B}(X)$ tem homologia zero. Para qualquer $p$, o grupo dos $p$ ciclos e dos $p$ bordos de $\mathcal{B}(X)$, são $B_{p}\left(X^{p}\right)+Z_{p}\left(X^{p-1}\right)$ e $B_{p}\left(X^{p}\right)$ respectivamente. Vejamos que $Z_{p}\left(X^{p-1}\right) \subset B_{p}\left(X^{p}\right)$. Segue do lema $(4.1 .7)$ que $H_{p}\left(X^{p-1}\right)=0$, ou seja que:

$$
Z_{p}\left(X^{p-1}\right)=B_{p}\left(X^{p-1}\right) \subset B_{p}\left(X^{p}\right)
$$

Se $X^{0} \neq \emptyset$, o quociente $q_{\sharp}: \mathcal{Z}(X) \rightarrow \mathcal{D}(X)$ preserva aumentação, portando induz também um isomorfismo em homologia reduzida. A naturalidade, segue do fato que (4.5) comuta a nível de cadeia.

Corolário 4.1.10. Se X é um espaço bem filtrado. Então, a homologia do complexo de cadeia associado à filtração de $X$, é naturalmente isomorfa à homologia singular de $X$.

\subsection{Número de Interseção}

No capítulo anterior definimos as noções de orientação e de número de interseção no sentido diferencial; nesta seção, definimos de novo tais conceitos empregando topologia algébrica pois para nosso caso este abordagem como veremos adiante é conveniente. Para a noção de número de interseção entre uma função diferenciável e uma subvariedade apresentamos o caso particular em que a função é definida num aberto da esfera $n$ dimensional o qual é suficiente para nosso interesse. Com respeito à noção de orientação que foi definida, é 
claro que a definição apresentada não faz sentido no caso de variedades topológicas pois neste caso não há espaço tangente; portanto, não é obvio generalizar a noção de orientação em tal caso. A idéia, é redefinir tal conceito empregando topologia algébrica; obtêm-se assim uma definição geral de orientação válida até no caso de variedades topologicas.

Seja $M$ uma variedade diferenciável (ou topológica) de dimensão $n$. Para todo $x \in M$ temos $H_{n}(M, M \backslash\{x\}) \simeq H_{n}\left(\mathbb{R}^{n}, \mathbb{R}^{n} \backslash\{0\}\right) \simeq \mathbb{Z}$

Definição 4.2.1. Uma orientação num ponto $x \in M$, é um gerador do grupo cíclico infinito $H_{n}(M, M \backslash\{x\})$.

Observe que como era de se esperar, em cada ponto $x \in M$ há exatamente dois orientações. Uma orientação para $M$, é definida como uma aplicação contínua que associa uma orientação a cada ponto $x \in M$. Vejamos os detalhes; definimos o conjunto:

$$
\mathcal{O}(M)=\bigcup_{x \in M}\{x\} \times H_{n}(M, M \backslash\{x\})
$$

obviamente há uma projeção natural $\pi: \mathcal{O}(M) \rightarrow M$.

Por uma seção em $\mathcal{O}(M)$ ao longo de um subconjunto $A \subset M$, é entendida uma aplicação $\tau: A \rightarrow \mathcal{O}(M)$ tal que $\pi \circ \tau$ é a inclusão de $A$ em $M$; se $A=M$, então $\tau$ é dita uma seção global em $\mathcal{O}(M)$. Vamos definir uma topologia no conjunto $\mathcal{O}(M)$, para isto é born considerar a seguinte observação.

\section{Observação 4.2 .2 .}

- Se $A, B \subset M$ e $B \subset A$, existe uma inclusão de $(M, M \backslash A)$ em $(M, M \backslash B)$. Denotamos por $\rho_{A B}: H_{n}(M, M \backslash A) \rightarrow H_{n}(M, M \backslash B)$ o homomorfismo induzido por tal inclusâo em homologia. Em particular, se $B=\{x\}$, simplesmente escrevemos $\rho_{A x}$.

- Se $A \subset M$ fixo, então toda classe de homologia $\alpha \in H_{n}(M, M \backslash A)$ induz uma seção $\mathcal{O}(\alpha ; A)$ em $\mathcal{O}(M)$ ao longo de $A$, definida como:

$$
\mathcal{O}(\alpha ; A)(x)=\rho_{A x}(\alpha), \quad \text { para cada } x \in A
$$

O seguinte resultado fornece uma topologia no conjunto $\mathcal{O}(M)$.

Proposição 4.2.3. O conjunto $\operatorname{Im}[\mathcal{O}(\alpha ; U)]$, em que $U$ percorre os subconjuntos abertos de $M$, e a percorre $H_{n}(M, M \backslash U)$, é uma base de conjuntos abertos para uma única topologia em $\mathcal{O}(M)$.

Lema 4.2.4. Sejam $A \subset M, \tau: A \rightarrow \mathcal{O}(M)$ uma seção em $\mathcal{O}(M)$ ao longo de A. Então, $\tau$ é contínua num ponto $x \in A$ se, e somente se, existem uma vizinhança aberta $U$ de $x$ em $M$ e uma classe de homologia $\alpha \in H_{n}(M, M \backslash U)$ tal que $\mathcal{O}(\alpha ; U)$ coincide com $\tau$ em $A \cap U$. 
Do lema anterior é inmediato que para cada subconjunto $A \subset M$ e toda classe de homologia $\alpha \in H_{n}(M, M \backslash A)$, a seção $\mathcal{O}(\alpha ; A)$ é contínua.

Definição 4.2.5. Uma orientação para uma variedade de dimensão n, é uma seção global contínua $\tau$; em $\mathcal{O}(M)$ tal que para cada $x \in M, \tau(x)$ é um gerador do grupo de homologia local $H_{n}(M, M \backslash\{x\})$. (i,e, pará cada $x \in M, \tau(x)$ é uma orientação para $M$ no ponto x) Se $M$ admite uma orientação, então $M$ é dita orientável; uma variedade junto com uma orientação é dita uma variedade orientada.

Observação 4.2.6. Se $U \subset M$ é um subconjunto aberto, então $\mathcal{O}(U)$ é aberto em $\mathcal{O}(M)$; além do mais, a topologia em $\mathcal{O}(U)$ coincide com a topologia induzida de $\mathcal{O}(M)$. Se $M$ é orientável, e $\tau: M \rightarrow \mathcal{O}(M)$ é uma orientação em $M$, então $\left.\tau\right|_{U}: U \rightarrow \mathcal{O}(U)$ é uma orientação para $U$.

Se $f: M \rightarrow N$, é definida uma aplicação $\mathcal{O}(f): \mathcal{O}(M) \rightarrow \mathcal{O}(N)$ com a condição que $\left.\mathcal{O}(f)\right|_{H_{n}(M, M \backslash\{x\})}$ coincide com o homomorfismo $f_{*}: H_{n}(M, M \backslash\{x\}) \rightarrow H_{n}(N, N \backslash\{f(x)\})$ induzido por $f$ em homologia para cada $x \in M$. É provado que se $f$ é um homeomorfismo, então $\mathcal{O}(f)$ é; além do mais, se $M$ e $N$ orientadas com $(M, \tau),\left(N, \tau^{\prime}\right), f$ é dito orientado positivo (o que preserva orientação) se $\mathcal{O}(f) \circ \tau=\tau^{\prime}$.

Casos particulares interesantes do feito acima para nosso objetivo são: O espaço Euclideano $\mathbb{R}^{n}$, e a esfera $S^{n}$. Se $M=\mathbb{R}^{n}$, para cada $v \in \mathbb{R}^{n}$, denotamos por $t_{v}$ a translação na direção do vetor $v, t_{v}: \mathbb{R}^{n} \rightarrow \mathbb{R}^{n}$, definida por $t_{v}(x)=x+v$. Obviamente, $t_{v}$ induz um isomorfismo em homologia $\left(t_{v}\right)_{*}: H_{n}\left(\mathbb{R}^{n}, \mathbb{R}^{n} \backslash\{0\}\right) \rightarrow H_{n}\left(\mathbb{R}^{n}, \mathbb{R}^{n} \backslash\{v\}\right)$. Neste caso, temos o seguinte resultado.

Proposição 4.2.7. Se $\mathcal{O}_{n}$ é um gerador do grupo cíclico infinito $H_{n}\left(\mathbb{R}^{n}, \mathbb{R}^{n} \backslash\{0\}\right)$, entâa, a aplicaçăo $\tau: \mathbb{R}^{n} \rightarrow \mathcal{O}\left(\mathbb{R}^{n}\right)$ definida por $\tau(x)=\left(t_{x}\right)_{*}\left(\mathcal{O}_{n}\right) \in H_{n}\left(\mathbb{R}^{n}, \mathbb{R}^{n} \backslash\{x\}\right)$ para cada $x \in \mathbb{R}^{n}$, é uma orientação para $\mathbb{R}^{n}$.

Segue da conexidade de $\mathbb{R}^{n}$ que qualquer orientação para $\mathbb{R}^{n}$ deve ser da forma da proposição acima; observe que se é escolhida uma orientação $\tau$ em $\mathbb{R}^{n}$, para algum gerador $\mathcal{O}_{n}$ do grupo $H_{n}\left(\mathbb{R}^{n}, \mathbb{R}^{n} \backslash\{0\}\right)$, então para cada $v \in \mathbb{R}^{n}, \mathcal{O}\left(t_{v}\right)$ leva $\tau(0)=\mathcal{O}_{n}$ em $\tau(v)$; portanto para cada $v \in \mathbb{R}^{n}$ a translação $t_{v}$ é orientada positiva. Mais geralmente temos:

Proposição 4.2.8. Seja $f: U \rightarrow V$ um difeomorfismo de classe $C^{1}$, em que $U, V \subset \mathbb{R}^{n}$ são abertos. Então $f$ é positivo (no sentido homológico) se e somente se o determinante de $\mathrm{d} f(x)$ é positivo para todo $x \in U$.

Observação 4.2.9. Se $M$ é uma variedade de dimensão zero, i.e., $M$ é um espaço topológico discreto, $\mathcal{O}(M)$ é também discreto, para cada $x \in M, U=\{x\}$ é aberto; dado $\alpha \in H_{0}(M, M \backslash\{x\}), \operatorname{Im}[\mathcal{O}(\alpha ; U)]=\{\alpha\}$. Portanto, toda seção $\tau: M \rightarrow \mathcal{O}(M)$ é contínua, e identificando com $1,-1$ os geradores do grupo cíclico infinito $H_{0}(M, M \backslash\{x\})$, 
fica claro que escolher uma orientação homológica numa variedade de dimensão zero, é o mesmo que escolher uma função arbitrária $\tau: M \rightarrow\{-1,1\}$.

Dado $x \in S^{n}, S^{n} \backslash\{x\}$ é contractil assim da seqüência exata do par $\left(S^{n}, S^{n} \backslash\{x\}\right)$, obtém-se um isomorfismo induzido pela inclusão $\widetilde{H}_{n}\left(S^{n}\right) \simeq H_{n}\left(S^{n}, S^{n} \backslash\{x\}\right)$; i.e., os homomorfismos $\rho_{S^{n} x}: H_{n}\left(S^{n}\right) \rightarrow H_{n}\left(S^{n}, S^{n} \backslash\{x\}\right)$ sảo isomorfismos para $n \geq 1$; e para $n=0$, a restrição de $\rho_{S^{n} x}$ a $\widetilde{H}_{n}\left(S^{n}\right)$ é um isomorfismo; daí, se $\alpha$ é um gerador do grupo cíclico infinito $\widetilde{H}_{n}\left(S^{n}\right), \mathcal{O}\left(\alpha ; S^{n}\right)$ é uma orientação para $S^{n}$. Assim, da conexidade da esfera temos que há uma correpondença $1-1$ :

$$
\text { \{geradores de } \left.\left.\widetilde{H}_{n}\left(S^{n}\right)\right\} \ni \alpha \mapsto \mathcal{O}\left(\alpha ; S^{n}\right) \in \text { orientaçōes de } S^{n}\right\},
$$

para $n \geq 1$, para $n=0$ vemos que $S^{0}=\{-1,1\}$ é discreto, portanto da observação acima temos que há 4 possíveis orientações em $S^{0}$; mas a imagem da aplicação injetiva (4.6) possue só dois delas (podem ser as que associam sinais distintos aos dois pontos de $S^{0}$ ), nos só estamos interesados em estas. Em resumo, identificamos as orientaçōes na esfera $S^{n}$ com os geradores do grupo $\widetilde{H}_{n}\left(S^{n}\right)$.

Vejamos que uma vez escolhido um gerador $\mathcal{O}_{n}$ para o grupo $H_{n}\left(\mathbb{R}^{n}, \mathbb{R}^{n} \backslash\{0\}\right)$, além de obter uma orientação no sentido homológico no espaço $\mathbb{R}^{n}$, obtemos uma correspondência canônica entre as orientações diferenciáveis e homológicas em variedades. De fato, seja $M$ uma variedade diferenciável de dimensão $n \geq 1$ orientada no sentido diferenciável; sejam $x \in M$ e $\varphi: U \rightarrow \widetilde{U}$ uma carta para $M \operatorname{com} x \in U$ e $0=\varphi(x)$. O isomorfismo entre espaços vetoriais $\mathrm{d} \varphi(x): T_{x} M \rightarrow \mathbb{R}^{n}$ induz uma bijeção entre o conjunto das orientações dos espaços vetoriais $T_{x} M$ e $\mathbb{R}^{n}$. Por outro lado, o isomorfismo de grupos:

$$
\varphi_{*}: H_{n}(M, M \backslash\{x\}) \longrightarrow H_{n}\left(\mathbb{R}^{n}, \mathbb{R}^{n} \backslash\{0\}\right)
$$

induz um bijeção entre o conjunto dos geradores do grupo cíclico infinito $H_{n}(M, M \backslash\{x\})$ e o conjunto dos geradores do grupo cíclico infinito $H_{n}\left(\mathbb{R}^{n}, \mathbb{R}^{n} \backslash\{0\}\right)$. Além do mais, temos uma bijeção canônica entre o conjunto das orintações para $\mathbb{R}^{n}$ como espaço vetorial e o conjunto dos geradores de $H_{n}\left(\mathbb{R}^{n}, \mathbb{R}^{n} \backslash\{0\}\right)$, a qual leva a orientação standard de $\mathbb{R}^{n}$ no gerador $\mathcal{O}_{n}$. Portanto, a carta $\varphi$ induz uma bijeção entre o conjunto das orientações para $T_{x} M$ (como espaço vetorial) e o conjunto dos geradores do grupo cíclico infinito $H_{n}(M, M \backslash\{x\})$ veja a flecha pontilhada no seguinte diagrama:

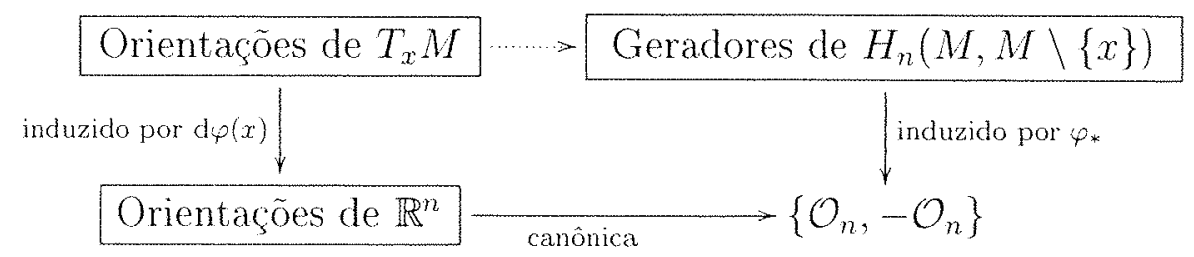

\footnotetext{
${ }^{1}$ Se $M$ tem dimensão zero, pela definição dada no sentido diferenciável, uma orientação em $M$ é simplesmente uma função arbitraria $\tau: M \rightarrow\{-1,1\}$, a qual portanto é compativel com a noçâo de orientação homológica.
} 
Segue da proposição 4.2.8 que a flecha pontilhada no diagrama acima não depende da $\operatorname{carta} \varphi$.

Proposição 4.2.10. Seja $M$ uma variedade de dimensão $n \geq 1$. Seja $\tau: M \rightarrow \mathcal{O}(M)$ uma seção global em $\mathcal{O}(M)$, tal que para cada $x \in M, \tau(x)$ é um gerador do grupo cíclico infinito $H_{n}(M, M \backslash\{x\})$, seja $\tilde{\tau}_{x}$ a orientação de $T_{x} M$ correspondente a $\tau(x)$ pela bijeção explicada acima. Então $\tau$ é contínua (i,e,. $\tau$ é uma orientação para $M$ no sentido homolôgico) se, e somente se, a familia $\tilde{\tau}=\left(\tilde{\tau}_{x}\right)_{x \in M}$ define uma orientação no sentido diferenciável para $M$.

Demonstração. Suponhamos que a familia $\tilde{\tau}$ define uma orientação no sentido diferenciável para $M$. Para cada $x \in M$, é possível achar uma carta $\varphi: U \rightarrow \widetilde{U}$ para $M \operatorname{com} x \in U$, $\varphi(x)=0$ a qual preserva orientação no sentido que para cada $y \in U$, o isomorfismo $\mathrm{d} \varphi(y): T_{y} M \rightarrow \mathbb{R}^{n}$ é positivo; i.e., para cada $y \in U, \mathrm{~d} \varphi(y)$ leva a orientação $\tilde{\tau}_{y}$ para $T_{y} M$, na orientação standard de $\mathbb{R}^{n}$; daí, o isomorfismo:

$$
\varphi_{*}: H_{n}(M, M \backslash\{y\}) \longrightarrow H_{n}\left(\mathbb{R}^{n}, \mathbb{R}^{n} \backslash\{\varphi(y)\}\right)
$$

leva $\tau(y)$ em $\tau^{[n]}(\varphi(y))$; em que denotamos por $\tau^{[n]}$ a orientaçāo homológica em $\mathbb{R}^{n}$ induzida por $\mathcal{O}_{n}$, temos então o seguinte diagrama comutativo:

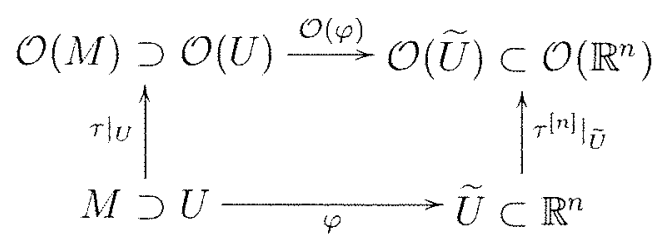

Como $\mathcal{O}(\varphi)$ é um homeomorfismo, $\left.\tau\right|_{U}$ é contínua, mas como $x$ arbitrario, temos que $\tau$ e contínua. Reciprocamente se $\tau$ é contínua, seja $x \in M$ e $\varphi: U \rightarrow \widetilde{U}$ carta em $M$ com $x \in U$ e $U$ conexo. Da conexidade de $U$, temos que $\varphi$ é um homeomorfismo positivo ou negativo (no sentido homolôgico); compondo $\varphi$ com um isomorfismo negativo se precisar, podemos supor sem perda de generalidade que $\varphi$ é orientado positivo, quer dizer que para cada $y \in U$, o isomorfismo (4.8) leva $\tau(y)$ em $\tau^{[n]}(\varphi(y))$; logo d $\varphi(y)$ leva $\tilde{\tau}_{y}$ em a orientação standard de $\mathbb{R}^{n}$. Ou seja que $\varphi$ é possitivo (obviamente no sentido diferenciável)

É claro pelo diagrama (4.7) e a proposição acima que a orientação homológica en $\mathbb{R}^{n}$ induzida por $\mathcal{O}_{n}$, corresponde à orientação diferenciável standard para $\mathbb{R}^{n}$.

Agora a pergunta é como escolher os $\mathcal{O}_{n}$ para cada $n$ ?

Para $n=0, \mathcal{O}_{0} \in H_{0}\left(\mathbb{R}^{0}, \mathbb{R}^{0} \backslash\{0\}\right)=H_{0}(\{0\})$ a escolha é canônica. Se $n \geq 1$, seja $\alpha_{n}: S^{n} \backslash\{\mathfrak{s}\} \rightarrow \mathbb{R}^{n}$ um difeomorfismo positivo em relação à orientação diferenciável determinada pelo vetor normal que aponta para fora e $\alpha_{n}(\mathfrak{n})=0$ (em que $\mathfrak{s}$ e $\mathfrak{n}$ denotam o polo sul e norte em $S^{n}$ respectivamente.) Suponhamos ter escolhido $\mathcal{O}_{k}$ para certo $k$, 
consideremos a seqüência de isomorfismos no seguinte diagrama:

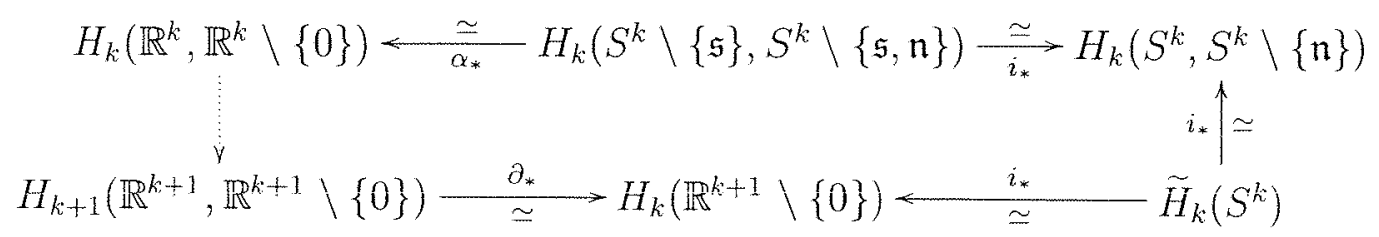

definimos $\mathcal{O}_{k+1}$ como sendo a imagem de $\mathcal{O}_{k}$ pelo isomorfismo no diagrama acima.

Observação 4.2.11. Considerando a seqüência exata do par $\left(\mathbb{R}^{k}, \mathbb{R}^{k} \backslash\{0\}\right)$, obtemos o seguinte isomorfismo: $H_{k}\left(\mathbb{R}^{k}, \mathbb{R}^{k} \backslash\{0\}\right) \stackrel{i_{*}^{-1} \circ \partial_{*}}{\longrightarrow} \widetilde{H}_{k}\left(S^{k-1}\right)$, portanto $i_{*}^{-1} \partial_{*}\left(\mathcal{O}_{k}\right)$ é um gerador do grupo $\widetilde{H}_{k}\left(S^{k-1}\right)$, afirmamos que a orientação homológica na esfera associada com $i_{*}^{-1} \partial_{*}\left(\mathcal{O}_{k}\right)$ corresponde à orientação diferenciável determinada pelo vetor normal que aponta para fora. Com efeito, basta provar que a orientação homológica na esfera correspondente com a orientação diferenciável determinada pelo vetor normal que aponta para fora councide com a orientação homológica na esfera associada com $i_{*}^{-1} \partial_{*}\left(\mathcal{O}_{k}\right)^{\prime}$ num ponto, por exemplo em $\mathfrak{n}$; observando o diagrama (4.7) para este caso, temos que bastará provar que $\alpha_{*}^{-1}\left(\mathcal{O}_{k-1}\right)=i_{*}^{-1} \partial_{*}\left(\mathcal{O}_{k}\right)$, mas isto é imediato do diagrama (4.9).

Na proposição 4.2 .10 provamos a equivalença entre as definições de orientação homológica e orientação diferenciável. Também, é possível provar que um difeomorfismo definido entre variedades direfenciáveis orientadas é positivo no sentido diferenciável se, e somente se, é orientado positivo no sentido homológico.

O seguinte é um resultado adaptado de idéias encontradas em [14].

Teorema 4.2.12. Dadas uma variedade diferenciável $M$ e uma subvariedade diferenciável fechada $N \operatorname{com} \operatorname{Cod}_{M}(N)=n$, e uma orientação transversa em $N$, então associamos um isomorfismo:

$$
H_{n}(M, M \backslash N) \stackrel{\varrho}{\longrightarrow} H_{0}(N)
$$

Além do mais, se $M^{\prime}$ é uma subvariedade de $M$ transversa a $N$, e $N^{\prime}=M^{\prime} \cap N$, então o seguinte diagrama:

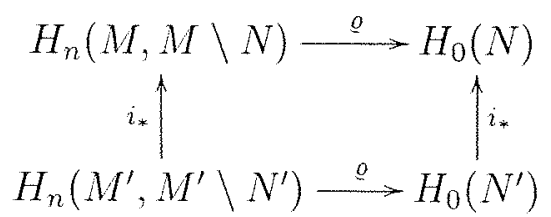

comuta. Como caso trivial temos se $N=\{a\} \operatorname{com} \operatorname{dim} M=n$, então o isomorfismo

$$
\underline{\varrho}: H_{n}(M, M \backslash\{a\}) \longrightarrow H_{0}(\{a\})
$$

leva o gerador associado à orientação diferenciável em $T_{a} M$, no gerador canônico de $H_{0}(\{a\})$. 
O isomorfismo $\varrho$ do teorema acima será utilizado ao longo deste texto; de agora em diante, a menos de menção em contrário, nos reportaremos a $\varrho$ como sendo tal isomorfismo. Também, denotamos por $\alpha^{[n]}=i_{*}^{-1} \partial_{*}\left(\mathcal{O}_{n+1}\right)$ o gerador de $\widetilde{H}_{n}\left(S^{n}\right)$, dado na observação (4.2.11). Vamos agora à definição desejada.

Definição 4.2.13. Sejam $U \subset S^{n}$ um subconjunto aberto, $M$ uma variedade diferenciável, $N \subset M$ uma subvariedade fechada transversalmente orientada tal que $\operatorname{Cod}_{M}(N)=n$. Seja $f: U \rightarrow M$ uma aplicação contínua tal que $f^{-1}(N)$ compacto. Definimos o número de interseção (no sentido homológico) da $f$ com a subvariedade $N$; como sendo o único inteiro $\eta(f, N)$ para o qual temos a igualdade:

$$
\phi\left(\alpha^{[n]}\right)=\eta(f, N)
$$

Em que o homomorfismo $\phi: \widetilde{H}_{n}\left(S^{n}\right) \rightarrow \mathbb{Z}$ é obtido pela composição das aplicaçôes no diagrama:

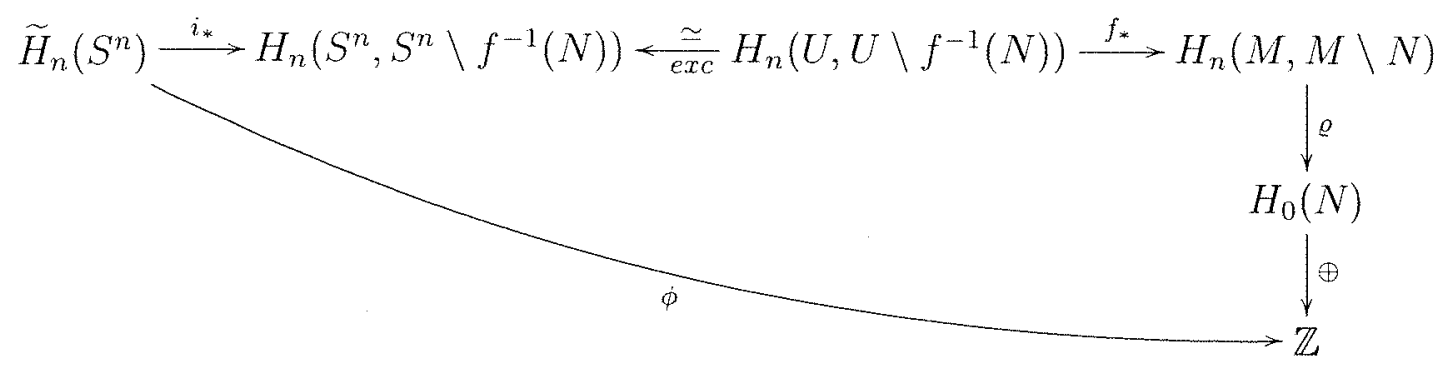

Observação 4.2.14. Se $K$ é um subconjunto compacto de $U$ contendo $f^{-1}(N)$, então no diagrama acima, é possível substituir $f^{-1}(N)$ por $K$ e obter um novo diagrama comutativo:

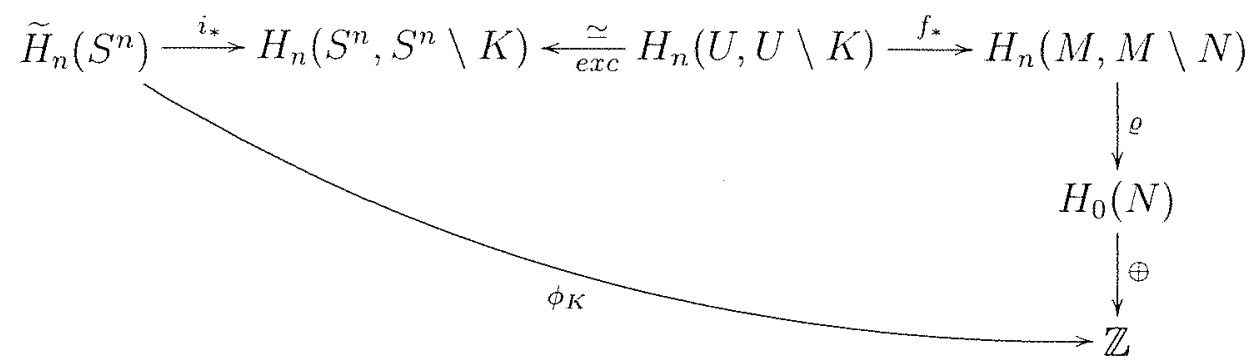

Como $f^{-1}(N) \subset K$, a função $f$ leva $U \backslash K$ em $M \backslash N$. Portanto, pode-se definir um número inteiro $\eta(f, N)_{K}$, como:

$$
\phi_{K}\left(\alpha^{[n]}\right)=\eta(f, N)_{K}
$$

Temos que $\eta(f, N)_{K}=\eta(f, N)$. De fato, isso segue da comutatividade do seguinte dia- 
grama:

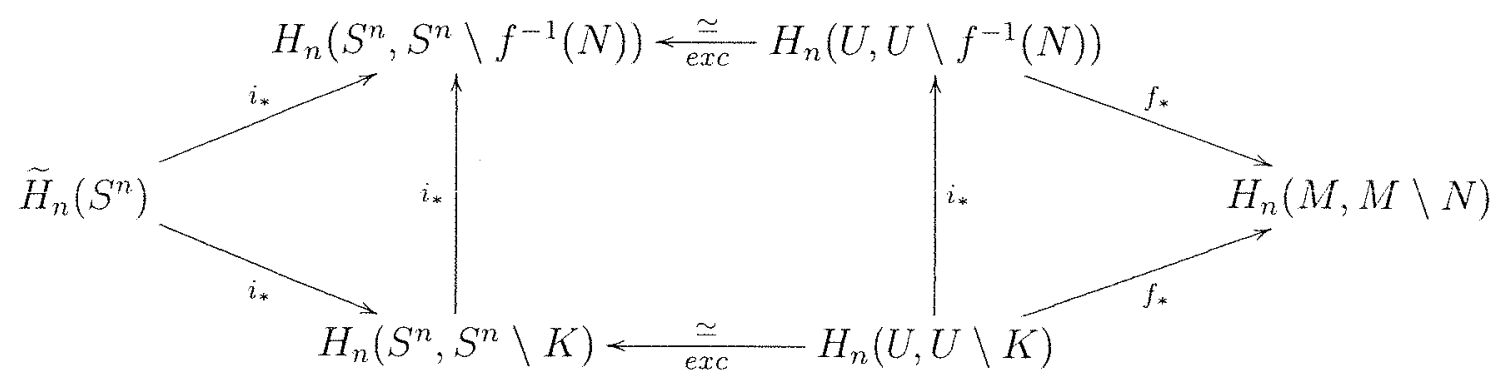

Alguns fatos básicos sobre o número de interseção são enunciadas no seguinte resultado.

Proposição 4.2.15. Sejam $f: U \subset S^{n} \rightarrow M$ e como na definição (4.2.13), então:

1. Se $V \subset U$ é aberto contendo $f^{-1}(N), \eta(f, N)=\eta\left(\left.f\right|_{V}, N\right)$;

2. Se $W$ é um aberto em $M$, tal que $f(U) \subset W$, então $\eta(f, N)=\eta(g, N \cap W)$, em que $g$ denota a função $f$ mudando o contradominio $M$ por $W$;

3. $\operatorname{Se} f(U) \cap N=\emptyset, \eta(f, N)=0$;

4. Se U é a união disjunta de abertos em $S^{n}, U=\bigcup_{\lambda \in L} U_{\lambda}$, então

$$
\eta(f, N)=\sum_{\lambda \in L} \eta\left(\left.f\right|_{U_{\lambda}}, N\right)
$$

5. Se $f$ é homotópica a uma aplicação $g: U \rightarrow M$, com homotopia $H$ tal que $H^{-1}(N)$ compacto, então $\eta(f, N)=\eta(g, N)$;

Demonstração. A prova do item (1) segue da comutatividade do diagrama:

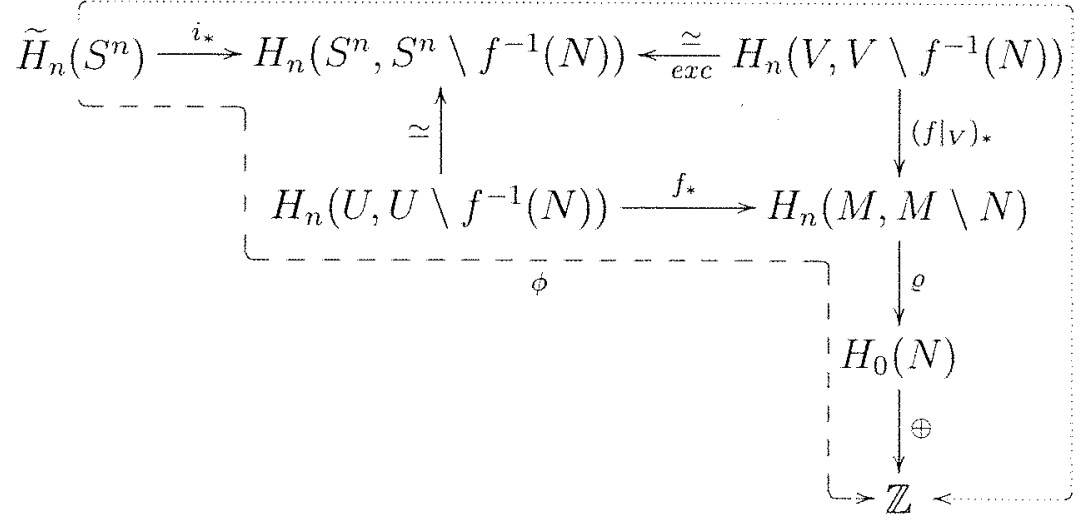


em que $\phi$ leva $\alpha^{[n]}$ em $\eta(f, N)$ e a linha pontilhada leva $\alpha^{[n]}$ em $\eta\left(\left.f\right|_{V}, N\right)$. A prova do item (2) segue da comutatividade do seguinte diagrama:

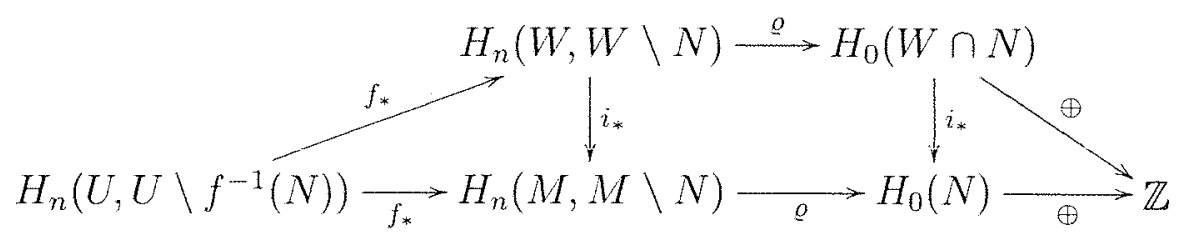

A prova do item (3) segue de observar que $f^{-1}(N)=\emptyset$ imlica que

$$
H_{n}\left(U, U \backslash f^{-1}(N)\right)=H_{n}(U, U)=0 .
$$

Para provar o item (4), como $f^{-1}(N)$ é compacto, a interseção $f^{-1}(N) \cap U_{\lambda}$ é não vazia só para um número finito de índices $\lambda \in L$. Dos items anteriores é possível deixar do lado os $\lambda^{\prime}$ s para os quais $f^{-1}(N) \cap U_{\lambda}=\emptyset$ e assumir que $L$ é finito. Consideramos o seguinte diagrama comutativo em que $f_{\lambda}=\left.f\right|_{U_{\lambda}}$.

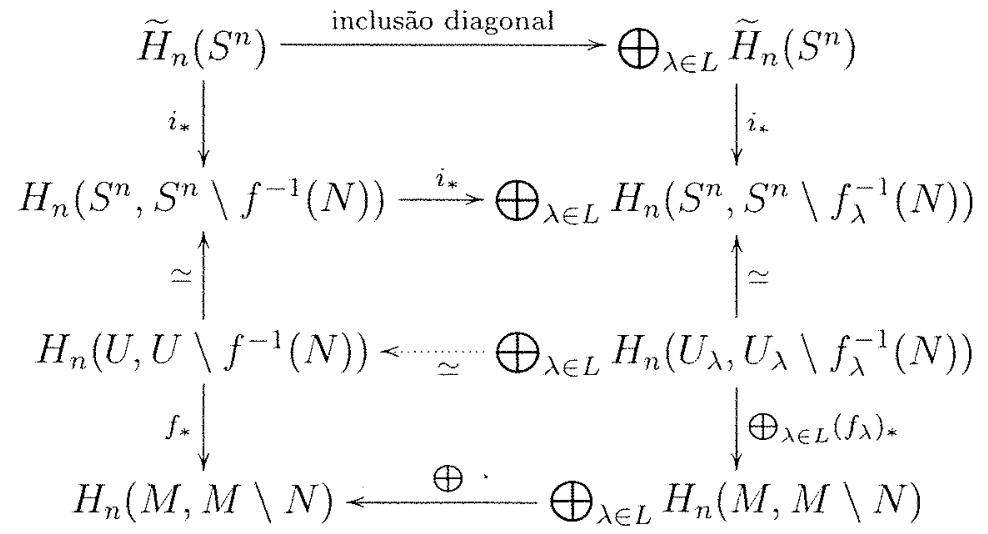

Para o item (5), denotamos por $K$ o compacto em $U$ dado pela projeção do compacto $H^{-1}(N) \subset U \times[0,1]$, então $f^{-1}(N) \subset K$ e $g^{-1}(N) \subset K$, pela observação (4.2.14), temos que $\eta(f, N)=\eta(f, N)_{K}$ e $\eta(g, N)=\eta(g, N)_{K}$. O resultado segue observando que $H$ define uma homotopia entre as aplicações de pares $f, g:(U, U \backslash K) \rightarrow(M, M \backslash N)$ e portanto os homomorfismos $f_{*}$ e $g_{*}$ empregados na definição de $\eta(f, N)$ e $\eta(g, N)$ são iguais.

O seguinte resultado mostra que as definições de número de interseção diferenciável e homológica são equivalentes.

Proposição 4.2.16. Se $f: U \subset S^{n} \rightarrow M$ é uma aplicação de classe $C^{1}$, com $U$ aberto. Seja $N \subset M$ uma subvariedade orientada transversalmente $e \operatorname{Cod}_{M}(N)=n$ tal que $f^{-1}(N)$ é compacto e $f \pitchfork N$. Então

$$
\eta(f, N)=\sum_{x \in f^{-1}(N)} \operatorname{sinal}(\mathrm{d} f(x))=\eta(f, N)_{d}
$$


Demonstração. Provemos inicialmente o caso em que $f^{-1}(N)=\{p\}$. Segue da observação (3.1.10) que d $f(p)$ é injetora, além do mais, $T_{f(p)} M=T_{f(p)} N \oplus \operatorname{Im}(\mathrm{d} f(p))$. É possível se precisar diminuir $U$ a um aberto tal que $f$ seja um mergulho, $\log \circ N^{\prime}=f(U) \subset M$ é uma subvariedade de $M$; além do mais, $M^{\prime}=N^{\prime} \cap N=\{f(p)\}$ e $T_{f(p)} M=T_{f(p)} N \oplus T_{f(p)} N^{\prime}$ portanto $N^{\prime} \pitchfork N$. Segue do teorema (4.2.12) e da funtorialidade da homologia singular que o seguinte diagrama:

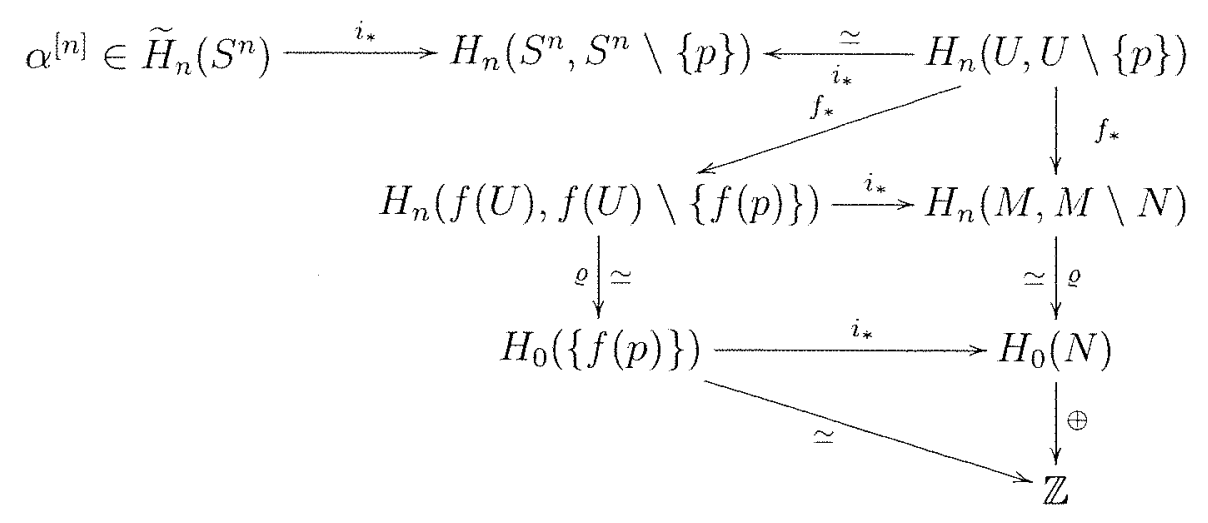

comuta. Como $\operatorname{Im}(\mathrm{d} f(p)) \simeq T_{f(p)} M^{\prime} \simeq T_{f(p)} M / T_{f(p)} N$ obtemos uma orientação no espaço $T_{f(p)} M^{\prime}=T_{f(p)} f(U)$. Logo o ponto $p$ é positivo se, e somente se, d $f(p)$ é positivo, se, e somente se, o isomorfismo obtido pela composta $T_{p}\left(S^{n}\right) \stackrel{\simeq}{\longrightarrow} T_{f(p)} M^{\prime} \stackrel{\simeq}{\longrightarrow} T_{f(p)} M / T_{f(p)} N$ é positivo se, e somente se, $T_{p}\left(S^{n}\right) \stackrel{\simeq}{\longrightarrow} T_{f(p)} M^{\prime}$ é positivo se, e somente se, $\varrho$ leva $f_{*}\left(\alpha^{[n]}\right)$ no gerador canônico de $H_{0}(\{f(p)\})$ se, e somente se, $f_{*}\left(\alpha^{[n]}\right)$ é o gerador de $H_{n}(f(U), f(U) \backslash\{f(p)\})$ associado à orientação diferenciável de $T_{f(p)} M^{\prime}$. Então é claro da comutatividade do digrama acima e das definições que $\eta(f, N)=\sin a l(\mathrm{~d} f(p))=\eta(f, N)_{d}$. Vamos agora ao caso geral, é claro das hipóteses que o conjunto $f^{-1}(N)$ é finito. Seja $f^{-1}(N)=\left\{x_{1}, \ldots, x_{s}\right\}$ podemos encontrar abertos disjuntos $\left(U_{i}\right)_{i=1}^{s}$ em $S^{n}$, tais que $x_{i} \in U_{i}$ para cada $i=1, \ldots, s . V=\bigcup_{i=1}^{s} U_{i}$ satisfaz as hipóteses do item 1 da proposição acima, e pelo item 4 temos que:

$$
\eta(f, N)=\eta\left(\left.f\right|_{V}, N\right)=\sum_{i=1}^{s} \eta\left(\left.f\right|_{U_{i}}, N\right) .
$$

A conclusão segue aplicando o caso demonstrado inicialmente para as funções $\left.f\right|_{U_{i}}$. 


\section{Capítulo 5}

\section{Isomorfismo Entre a Homologia de Morse e a Homologia Singular}

\subsection{O Teorema Fundamental}

O teorema central deste trabalho é o seguinte:

Teorema 5.1.1. Seja $f: M \rightarrow \mathbb{R}$ uma função de Morse definida numa variedade compacta $M$ e satisfazendo a condição de Morse-Smale de ordem $k=1$. Então:

1. A seqüência $\left(\mathcal{C}_{k}(f), \partial_{k}\right)_{k \in Z}$ é um complexo de cadeia, i.e., $\partial_{k-1} \circ \partial_{k}=0$;

2. A homologia do complexo $\left(\mathcal{C}_{k}(f), \partial_{k}\right)_{k \in Z}$ é isomorfa à homologia singular com coeficientes inteiros da variedade $M$.

No restante deste capítulo demonstraremos o teorema 5.1.1. Precisaremos de diversos resultados preliminares. Por estética e prática empregaremos com muita frequiência diagramas comutativos para visualizar a igualdade da composta de homomorfismos.

Lema 5.1.2. Sejam $f: M \rightarrow \mathbb{R}$ uma aplicação diferenciável e $p \in$ Crit $_{c}$ não-degenerado, $\operatorname{com} n_{-}(p)=k$. Então, a inclusão $i:\left(\mathcal{W}_{u}(p), \mathcal{W}_{u}(p) \backslash\{p\}\right) \rightarrow\left(f^{c}, f^{c} \backslash\{p\}\right)$ induz um isomorfismo em homologia.

Demonstração. Seja $\varphi: U \rightarrow \widetilde{U}$ uma carta de Morse em $M$ para $p$, tal que $\varphi(p)=0$ e $\operatorname{d} \varphi(p)\left(T_{p}\left(\mathcal{W}_{u}(p)\right)\right)=\mathbb{R}^{k} \times\{0\}$. Consideremos a projeção $\pi_{1}: \mathbb{R}^{k} \times \mathbb{R}^{n-k} \rightarrow \mathbb{R}^{k}$, é claro que $\mathrm{d}\left(\left.\pi_{1}\right|_{\varphi\left(u \cap \mathcal{W}_{u}(p)\right)}\right)(0)$ é a identidade em $\mathbb{R}^{k}$; segue do teorema da função inversa que $\pi_{1} l_{\varphi\left(U \cap \mathcal{W}_{u}(p)\right)}$ é um difeomorfismo numa vizinhança da origem $\varphi(p)=0$. Diminuindo 
$U$ se precisar, podemos supor que a restrição $\left.\pi_{1}\right|_{\varphi\left(U \cap \mathcal{W}_{u}(p)\right)}$ é um difeomorfismo. Seja $\psi=\pi_{2} \circ\left[\left.\pi_{1}\right|_{\varphi\left(U \cap \mathcal{W}_{u}(p)\right)}\right]^{-1}: V \subset \mathbb{R}^{k} \rightarrow \mathbb{R}^{n-k}$; então, $\varphi\left(U \cap \mathcal{W}_{u}(p)\right)$ coincide com o gráfico da função $\psi, \operatorname{Gr}(\psi)$. Mas, $\varphi\left(U \cap f^{c}\right)=\left\{(x, y) \in \mathbb{R}^{k} \times \mathbb{R}^{n-k}:\|x\| \geq\|y\|\right\} \cap \widetilde{U}$, e contém o conjunto $\operatorname{Gr}(\psi)$. Observe que $(\operatorname{Gr}(\psi), \operatorname{Gr}(\psi) \backslash\{0\})$ é um retrato por deformação forte de $\left(\varphi\left(U \cap f^{c}\right) \cap\left(V \times \mathbb{R}^{n-k}\right), \varphi\left(U \cap f^{c}\right) \cap\left(V \times \mathbb{R}^{n-k}\right) \backslash\{0\}\right)$ basta só definir a função:

$$
\mathcal{F}((x, y), t)=(x,(1-t) y+t \psi(y)) .
$$

Portanto, a inclusão:

$\left(\varphi\left(U \cap \mathcal{W}_{u}(p)\right), \varphi\left(U \cap \mathcal{W}_{u}(p)\right) \backslash\{0\}\right) \longrightarrow\left(\varphi\left(U \cap f^{c}\right) \cap\left(V \times \mathbb{R}^{n-k}\right), \varphi\left(U \cap f^{c}\right) \cap\left(V \times \mathbb{R}^{n-k}\right) \backslash\{0\}\right)$

induz um isomorfismo em homologia, logo compondo com $\varphi^{-1}$, temos que a inclusão $\left(U \cap \mathcal{W}_{u}(p), U \cap \mathcal{W}_{u}(p) \backslash\{p\}\right) \rightarrow\left(U \cap f^{c}, U \cap f^{c} \backslash\{p\}\right)$ induz um isomorfismo em homologia; o resultado desejado segue por excisão.

Pelo comentário feito na observação (3.4.3), podemos assumir que a função $f$ em questão é auto-indexante. Nossa estratégia será a de construir para cada $k$ um isomorfismo $\rho: \mathcal{C}_{k}(f) \rightarrow H_{k}\left(f^{k}, f^{k-1}\right)$ de modo que o diagrama:

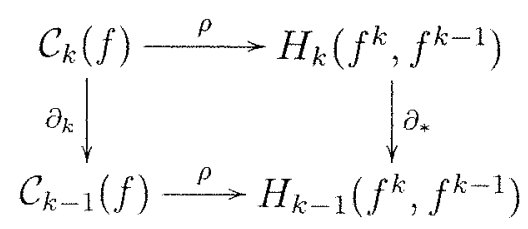

comuta.

Do lema 5.1.2, da observação 4.1 .2 e do lema 3.2 .13 obtemos o seguinte diagrama de isomorfismos:

$$
\begin{gathered}
\bigoplus_{p \in \mathrm{Crit}_{k}} H_{i}\left(\mathcal{W}_{u}(p), \mathcal{W}_{u}(p) \backslash\{p\}\right) \longrightarrow \bigoplus_{p \in \mathrm{Crit}_{k}} H_{i}\left(f^{k}, f^{k} \backslash\{p\}\right) \\
\uparrow \simeq \\
H_{i}\left(f^{k}, f^{k} \backslash \mathrm{Crit}_{k}\right) \\
H_{i}\left(f^{k}, f^{k-1}\right)
\end{gathered}
$$

Como $H_{i}\left(\mathcal{W}_{u}(p), \mathcal{W}_{u}(p) \backslash\{p\}\right)=0$ para $i \neq k$, segue do diagrama acima que $\left(f^{k}\right)_{k \in \mathbb{N}} \dot{e}$ uma boa filtração de $M$. Segue então do corolário 4.1.10 que a homologia do complexo de cadeia associado à filtração $\left(f^{k}\right)_{k \in \mathbb{N}}$ é isomorfa à homologia singular de $M$. Escolha uma orientação em $T_{p}\left(\mathcal{W}_{u}(p)\right)$ para todo $p \in$ Crit $(f)$; tal orientação nos define um gerador $\mathcal{O}_{p}$ do grupo infinito cíclico $H_{k}\left(\mathcal{W}_{u}(p), \mathcal{W}_{u}(p) \backslash\{p\}\right)$. Obtemos portanto um isomorfismo:

$$
\mathcal{C}_{k}(f) \stackrel{\simeq}{\longrightarrow} \bigoplus_{p \in \mathrm{Crit}_{k}} H_{k}\left(\mathcal{W}_{u}(p), \mathcal{W}_{u}(p) \backslash\{p\}\right)
$$


A composição de (5.3) com o isomorfismo definido pela flecha pontilhada no diagrama (5.2) nos fornece um isomorfismo $\rho: \mathcal{C}_{k}(f) \rightarrow H_{k}\left(f^{k}, f^{k-1}\right)$, para cada $k$. Se mostrarmos que o diagrama (5.1) comuta, ficará estabelecido que $\left(\mathcal{C}_{k}(f), \partial_{k}\right)_{k \in \mathbb{Z}}$ é um complexo de cadeia isomorfo ao complexo de cadeia associado à filtração $\left(f_{k}\right)_{k \in \mathbb{N}}$ de $M$. Isso completará a prova do teorema 5.1.1.

Dedicamos agora o resto deste capítulo à demonstração da comutatividade do diagrama (5.1). Precisamos provar que para cada $p \in \mathrm{Crit}_{k}, \partial_{k}(p)=\rho^{-1} \partial_{*} \rho(p)$; bastará mostrar que para cada ponto crítico $q \in$ Crit $_{k-1}$,

$$
\hat{q}\left(\partial_{k}(p)\right)=\hat{q}\left(\rho^{-1} \partial_{*} \rho(p)\right)
$$

em que $\hat{q}: \operatorname{Crit}_{k-1}(f) \rightarrow \mathbb{Z}$ é a aplicação que dá o coeficiente em $q$.

Lema 5.1.3. Se $q \in \mathrm{Crit}_{k-1}$, o seguinte diagrama

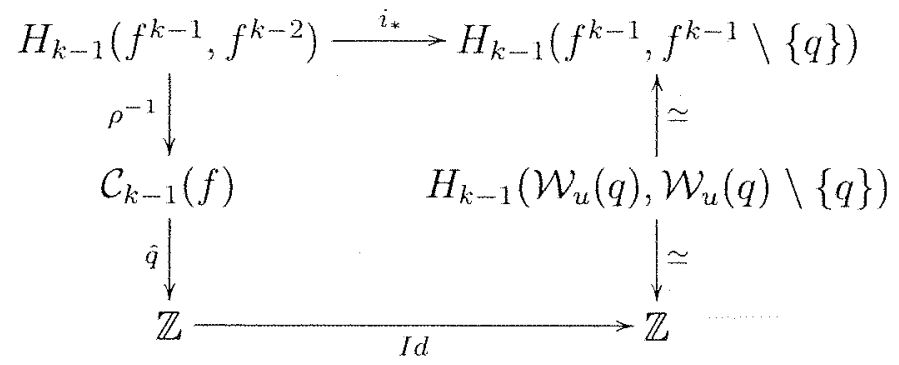

comuta, em que o isomorfismo entre $\mathbb{Z}$ e $H_{k-1}\left(\mathcal{W}_{u}(q), \mathcal{W}_{u}(q) \backslash\{q\}\right)$ é associado à orientaçäo escolhida em $\mathcal{W}_{u}(q)$, i.e., levando $\mathcal{O}_{q}$ em 1 .

Demonstração. Segue diretamente da definição de $\rho$ (olhe para o diagrama (5.2) trocando $k$ por $k-1$ e removendo as somas diretas).

\section{Observação 5.1 .4 .}

- Se $p \in \mathrm{Crit}_{k}$, pela forma como foi definido o isomorfismo $\rho$, o homomorfismo $f_{1}$ definido pelo diagrama abaixo:

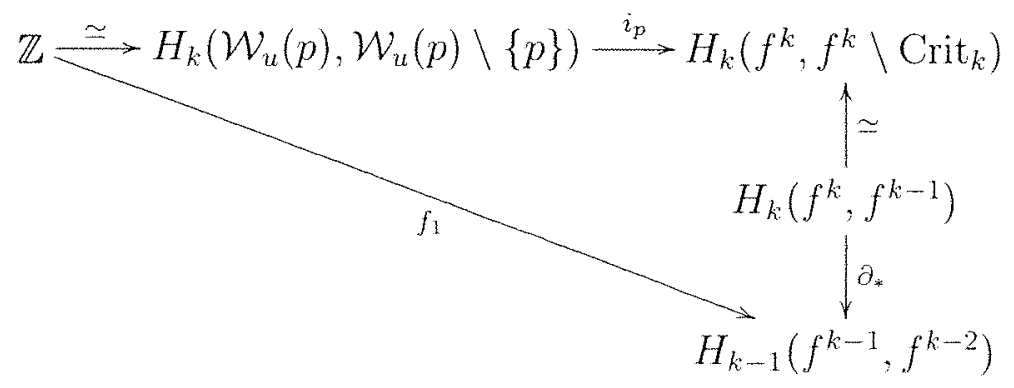

(em que o isomorfismo entre $\mathbb{Z}$ e $H_{k}\left(\mathcal{W}_{u}(p), \mathcal{W}_{u}(p) \backslash\{p\}\right)$ é associado à orientação escolhida em $\left.\mathcal{W}_{u}(p)\right)$ leva 1 em $\partial_{*} \rho(p)$. 
- Se $q \in \mathrm{Crit}_{k-1}$, segue do lema (5.1.3) que o homomorfismo $f_{3}$ definido pelo diagrama abaixo:

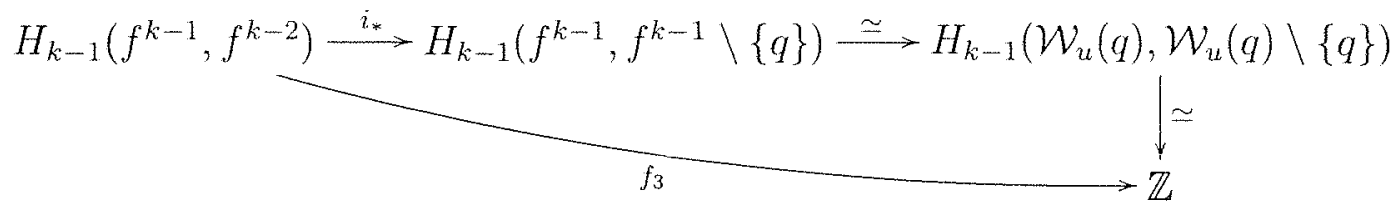

(em que o isomorfismo entre $\mathbb{Z}$ e $H_{k-1}\left(\mathcal{W}_{u}(q), \mathcal{W}_{u}(q) \backslash\{q\}\right)$ é associado à orientação escolhida em $\left.\mathcal{W}_{u}(q)\right)$ coincide com $\hat{q} \rho^{-1}$.

- Dos items anteriores, temos que a composta $f_{3} \circ f_{1}$ leva 1 em $\hat{q}\left(\rho^{-1} \partial_{*} \rho(p)\right)$.

Observação 5.1.5. Se $p \in \mathrm{Crit}_{k}$, levando em conta que $\mathcal{W}_{u}(p)$ é contrátil (vide observação 2.4.6), da seqüência em homologia do $\operatorname{par}\left(\mathcal{W}_{u}(p), \mathcal{W}_{u}(p) \backslash\{p\}\right)$, temos que o homomorfismo:

$$
H_{k}\left(\mathcal{W}_{u}(p), \mathcal{W}_{u}(p) \backslash\{p\}\right) \stackrel{\partial_{*}}{\longrightarrow} \widetilde{H}_{k-1}\left(\mathcal{W}_{u}(p) \backslash\{p\}\right)
$$

é um isomorfismo. Também se $k-1<a<k$, temos que $\mathcal{W}_{u}(p) \cap f^{-1}(a)$ é um retrato por deformação de $\mathcal{W}_{u}(p) \backslash\{p\}$, logo o homomorfismo:

$$
\widetilde{H}_{k-1}\left(\mathcal{W}_{u}(p) \cap f^{-1}(a)\right) \stackrel{i_{*}}{\longrightarrow} \widetilde{H}_{k-1}\left(\mathcal{W}_{u}(p) \backslash\{p\}\right)
$$

induzido pela inclusão é um isomorfismo. Se $k-1<a<a^{\prime}<k$ eq $\in \mathrm{Crit}_{k-1}$, então o homomorfismo

$$
H_{k}\left(f^{-1}\left(\left(-\infty, a^{\prime}\right)\right)\right) \stackrel{i_{*}}{\longrightarrow} H_{k}\left(f^{k} \backslash \mathrm{Crit}_{k}\right)
$$

induzido pela inclusão é um isomorfismo. Isso segue do fato que $f^{-1}\left(\left(-\infty, a^{\prime}-\epsilon\right]\right)$ é um retrato por deformação de ambos os conjuntos $f^{k} \backslash \mathrm{Crit}_{k}$ e $f^{-1}\left(\left(-\infty, a^{\prime}\right)\right)$ (vide lema 3.2.13).

Observação 5.1.6. Se $k-1<a<k$ e $p \in$ Crit $_{k}$, escolhemos um homeomorfismo $\varphi: \mathcal{W}_{u}(p) \rightarrow \mathbb{R}^{k}$ que leva $\mathcal{W}_{u}(p) \cap f^{-1}([a, k])$ sobre a bola unitária fechada de $\mathbb{R}^{k}$, leva $\mathcal{W}_{u}(p) \cap f^{-1}(a)$ sobre a esfera unitária de $\mathbb{R}^{k}$ e tal que $\varphi(p)=0$ (vide observação 2.4.6). Podemos escolher $\varphi$ de modo que o isomorfismo em homologia $\varphi_{*}$ leva o gerador do grupo $H_{k}\left(\mathcal{W}_{u}(p), \mathcal{W}_{u}(p) \backslash\{p\}\right), \mathcal{O}_{p}$ associado à orientação diferenciável escolhida em $T_{p}\left(\mathcal{W}_{u}(p)\right)$, em $\mathcal{O}_{k}$; observando o seguinte diagrama comutativo:

$$
\begin{gathered}
\widetilde{H}_{k}\left(\mathcal{W}_{u}(p), \mathcal{W}_{u}(p) \backslash\{p\}\right) \stackrel{\varphi_{*}}{\longrightarrow} H_{k}\left(\mathbb{R}^{k}, \mathbb{R}^{k} \backslash\{0\}\right) \\
\simeq \downarrow_{i_{*}^{-1} \circ \partial_{*}} \\
i_{*}^{-1} \circ \partial_{*} \downarrow \simeq \\
\widetilde{H}_{k-1}\left(\mathcal{W}_{u}(p) \cap f^{-1}(a)\right) \stackrel{\varphi_{*}}{\longrightarrow} \widetilde{H}_{k-1}\left(S^{k-1}\right)
\end{gathered}
$$

vemos que a imagem de $\mathcal{O}_{p}$ pelo isomorfismo $i_{*}^{-1} \partial_{*}$ é o gerador do grupo cúclico infinito $\widetilde{H}_{k-1}\left(\mathcal{W}_{u}(p) \cap f^{-1}(a)\right)$ levado por $\left(\varphi_{a}\right)_{*}$, em $i_{*}^{-1} \partial_{*}\left(\mathcal{O}_{k}\right)$ (vide (4.2.11)). Portanto a orientação homológica determinada em $\mathcal{W}_{u}(p) \cap f^{-1}(a)$ pela imagem de $\mathcal{O}_{p}$ corresponde à orientação na esfera $S^{k-1}$ determinada pelo vetor normal que aponta para fora. 
Pelo comentário depois da definição do complexo de Morse-Witten, temos que dado $a \in \mathbb{R} \operatorname{com} k-1<a<k, \hat{q}\left(\partial_{k}(p)\right)$ é igual ao número de interseção de $f^{-1}(a) \cap \mathcal{W}_{u}(p)$ com $f^{-1}(a) \cap \mathcal{W}_{s}(q)$ em $f^{-1}(a)$. Portanto, da definição de número de interseção e da observação 5.1.4, resta provar que o seguinte diagrama:

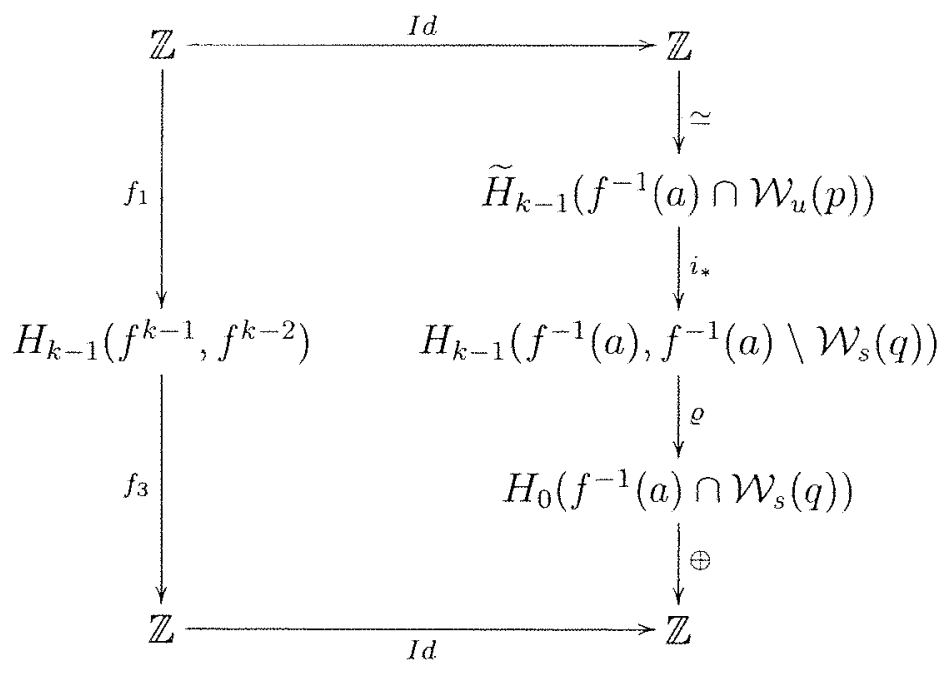

comuta, em que o isomorfismo $\mathbb{Z} \simeq \widetilde{H}_{k-1}\left(f^{-1}(a) \cap \mathcal{W}_{u}(p)\right)$ é associado à orientação em $f^{-1}(a) \cap \mathcal{W}_{u}(p)$ determinada pela imagem do gerador $\mathcal{O}_{p}$ pelo isomorfismo $i_{*}^{-1} \partial_{*}$ (vide o diagrama (5.5) e o comentário posterior). Além do mais, o isomorfismo $\varrho$ tem sinal definido pela orientação transversa de $\mathcal{W}_{s}(q)$ em $M$.

Agora que temos claro o que precisamos provar no final das contas, vamos fixar mais uma notação para facilitar o desenvolvimento da prova.

Seja $a^{\prime} \in \mathbb{R}$, fixo tal que $k-1<a<a^{\prime}<k$, denotamos por (S) o conjunto $f^{-1}\left(\left(-\infty, a^{\prime}\right)\right) \backslash\left(f^{-1}\left(\left(-\infty, a^{\prime}\right)\right) \cap \mathcal{W}_{s}(q)\right)$.

\section{Observação 5.1.7.}

- É claro pela forma como foi escolhido $a^{\prime}$, que as hipóteses do teorema (4.2.12) são satisfeitas para $M=f^{-1}\left(\left(-\infty, a^{\prime}\right)\right)$ e $N=f^{-1}\left(\left(-\infty, a^{\prime}\right)\right) \cap \mathcal{W}_{s}(q)$. Portanto, temos um isomorfismo:

$$
H_{k-1}\left(f^{-1}\left(\left(-\infty, a^{\prime}\right)\right),(S) \stackrel{\varrho}{\longrightarrow} H_{0}\left(f^{-1}\left(\left(-\infty, a^{\prime}\right)\right) \cap \mathcal{W}_{s}(q)\right)\right.
$$

Além do mais (tomando $M^{\prime}=f^{-1}($ a) no teorema 4.2.12), temos que o diagrama:

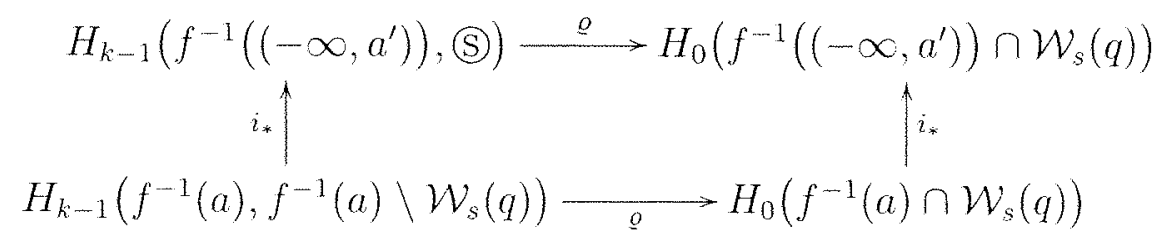

comuta. 
- O conjunto $f^{-1}\left(\left(-\infty, a^{\prime}\right)\right) \cap \mathcal{W}_{s}(q)$ é homeomorfo a um disco aberto, logo é conexo e portanto $H_{0}\left(f^{-1}\left(\left(-\infty, a^{\prime}\right)\right) \cap \mathcal{W}_{s}(q)\right) \simeq \mathbb{Z}$.

Considere os homomorfismos $f_{2}, f_{4}$ e $f_{5}$ definidos pelos diagramas abaixo:

$$
\begin{gathered}
\mathbb{Z} \stackrel{\simeq}{\longrightarrow} \widetilde{H}_{k-1}\left(f^{-1}(a) \cap \mathcal{W}_{u}(p)\right) \stackrel{i_{*}}{\longrightarrow} H_{k-1}\left(f^{-1}\left(\left(-\infty, a^{\prime}\right)\right),(\mathrm{S})\right) \\
H_{k-1}\left(f^{-1}\left(\left(-\infty, a^{\prime}\right)\right),(\mathrm{S}) \stackrel{o}{\longrightarrow} H_{0}\left(f^{-1}\left(\left(-\infty, a^{\prime}\right)\right) \cap \mathcal{W}_{s}(q)\right) \stackrel{\simeq}{\longrightarrow} \mathbb{Z}\right. \\
H_{k-1}\left(f^{-1}(a), f^{-1}(a) \backslash \mathcal{W}_{s}(q)\right) \stackrel{\varrho}{\longrightarrow} H_{0}\left(f^{-1}(a) \cap \mathcal{W}_{s}(q)\right) \stackrel{\oplus}{\longrightarrow} \mathbb{Z}
\end{gathered}
$$

Observamos de novo que o isomorfismo $\mathbb{Z} \stackrel{\simeq}{\longrightarrow} \widetilde{H}_{k-1}\left(f^{-1}(a) \cap \mathcal{W}_{u}(p)\right)$ que aparece em (5.7), é associado à orientação em $f^{-1}(a) \cap \mathcal{W}_{u}(p)$ determinada pela imagem do gerador $\mathcal{O}_{p}$ pelo isomorfismo $i_{*}^{-1} \partial_{*}$ (vide o diagrama 5.5 e o comentario posterior). O isomorfismo $\varrho$ que aparece em (5.8) tem sinal definido pela orientação transversa de $\mathcal{W}_{s}(q)$ em $M$. E o isomorfismo $\varrho$ que aparece em (5.9), é associado à orientação transversa de $\mathcal{W}_{s}(q) \cap f^{-1}(a)$ em $f^{-1}(a)$ (vide (3.10))

Lema 5.1.8. O diagrama:

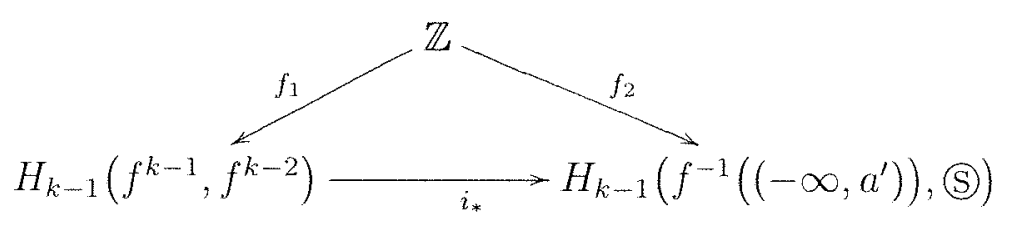

comuta.

Demonstração. A comutatividade do diagrama no lema, segue da comutatividade do di- 
agrama:

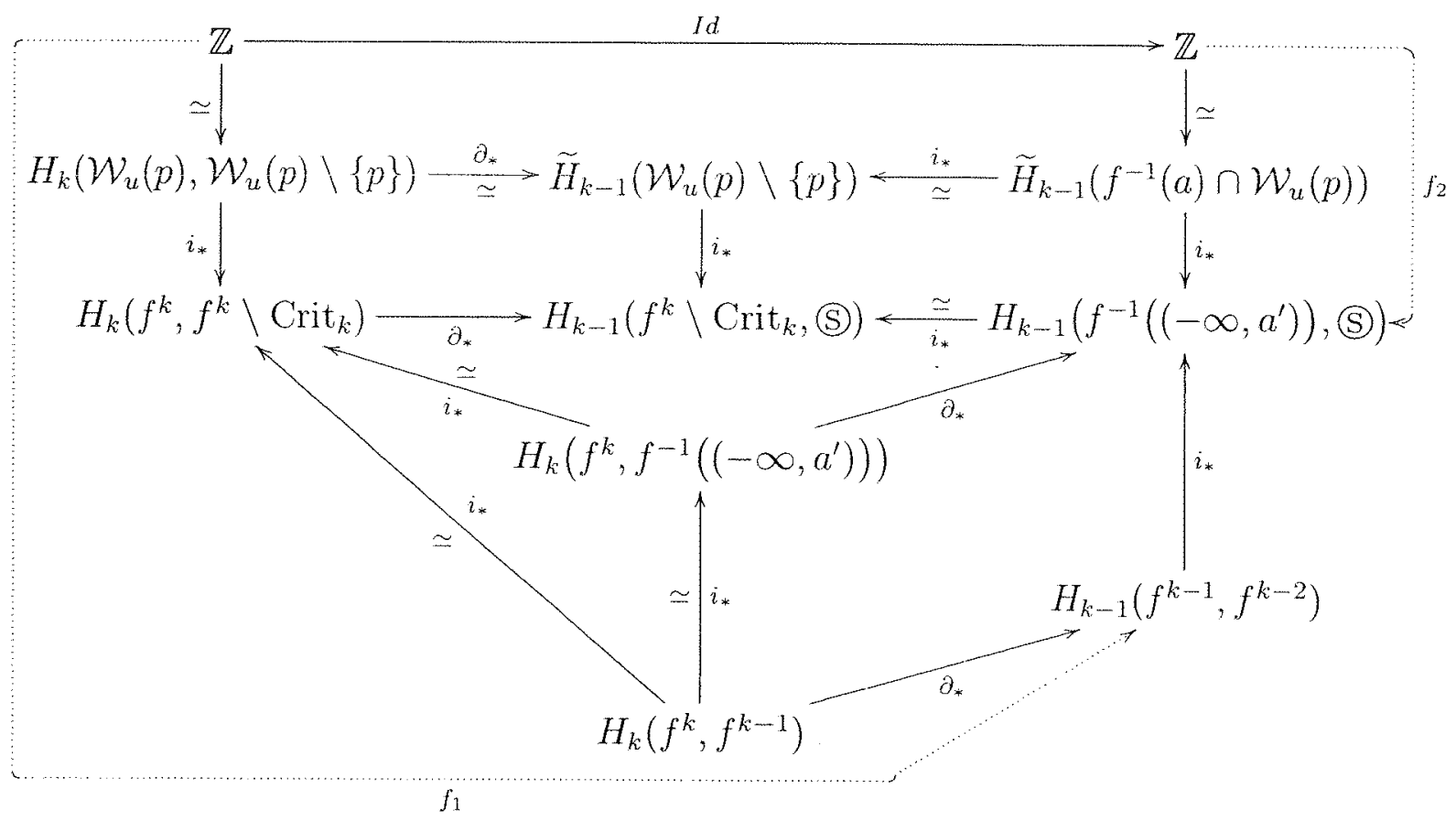

A comutatividade do retângulo superior do diagrama acima segue da forma pelas quais os geradores dos grupos infinitos cíclicos $H_{k}\left(\mathcal{W}_{u}(p), \mathcal{W}_{u}(p) \backslash\{p\}\right)$ e $\widetilde{H}_{k-1}\left(f^{-1}(a) \cap \mathcal{W}_{u}(p)\right)$ foram escolhidos (vide observação 5.1.6).

Lema 5.1.9. O diagrama:

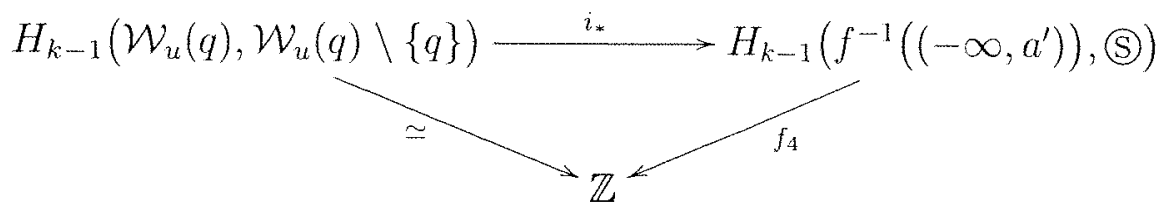

comuta, em que o isomorfismo $H_{k-1}\left(\mathcal{W}_{u}(q), \mathcal{W}_{u}(q) \backslash\{q\}\right) \simeq \mathbb{Z}$ é associado à orientação de $\mathcal{W}_{u}(q)$

Demonstração. Como $n_{-}(q)=k-1$, a variedade $\mathcal{W}_{u}(q)$ é homeomorfa a $\mathbb{R}^{k-1}$ e portanto podemos identificá-la com um aberto na esfera $S^{k-1}$ (via projeção estereográfica, por exemplo). Observe também que $\mathcal{W}_{s}(q) \cap f^{-1}\left(\left(-\infty, a^{\prime}\right)\right)$ tem codimensão $k-1$ em 
$f^{-1}\left(\left(-\infty, a^{\prime}\right)\right)$. Então o diagrama:

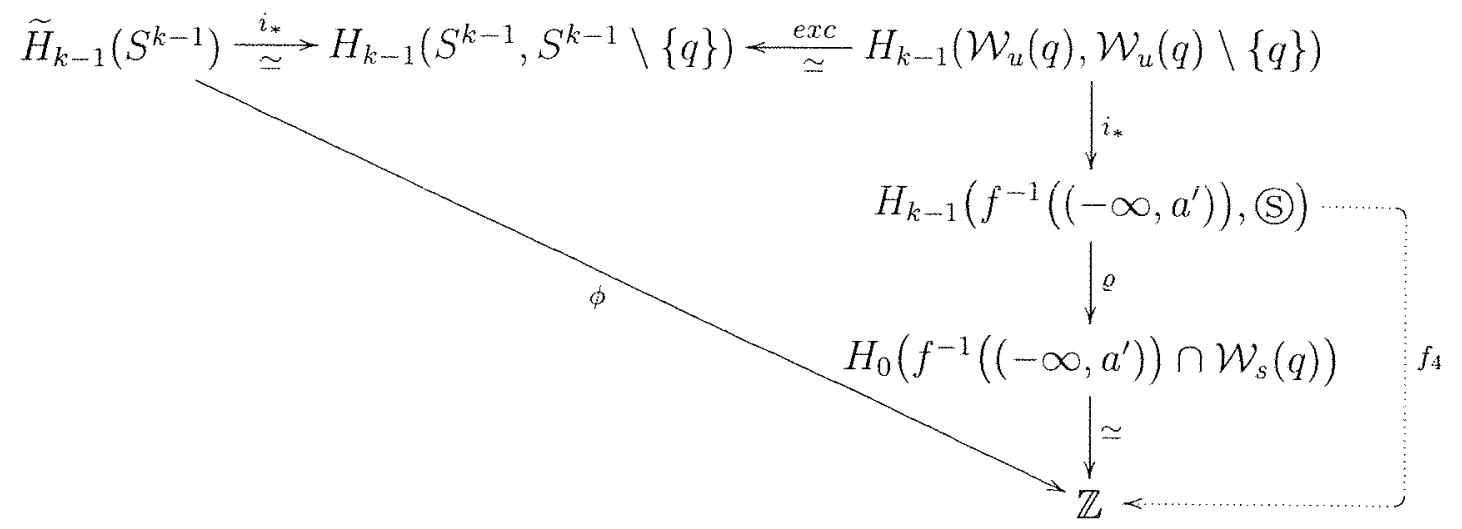

calcula o número de interseção da aplicação inclusão:

$$
S^{k-1} \supset \mathcal{W}_{u}(q) \longrightarrow f^{-1}\left(\left(-\infty, a^{\prime}\right)\right)
$$

com a subvariedade $\mathcal{W}_{s}(q) \cap f^{-1}\left(\left(-\infty, a^{\prime}\right)\right)$ de $f^{-1}\left(\left(-\infty, a^{\prime}\right)\right)$; obviamente, esse número de interseção é igual a 1 . Portanto a composta $f_{4} \circ i_{*}$ na coluna direita do diagrama leva $\mathcal{O}_{q}$ em 1 .

Lema 5.1.10. O diagrama:

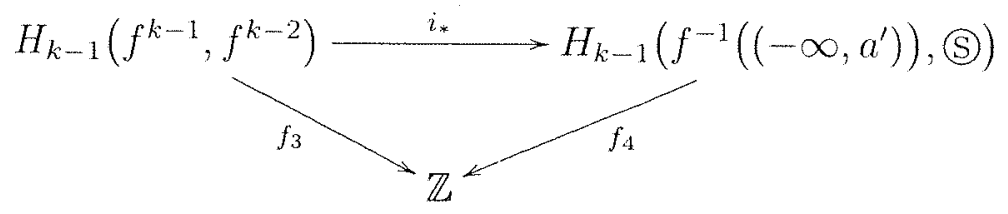

comuta.

Demonstração. A comutatividade do diagrama no lema segue da comutatividade do diagrama:

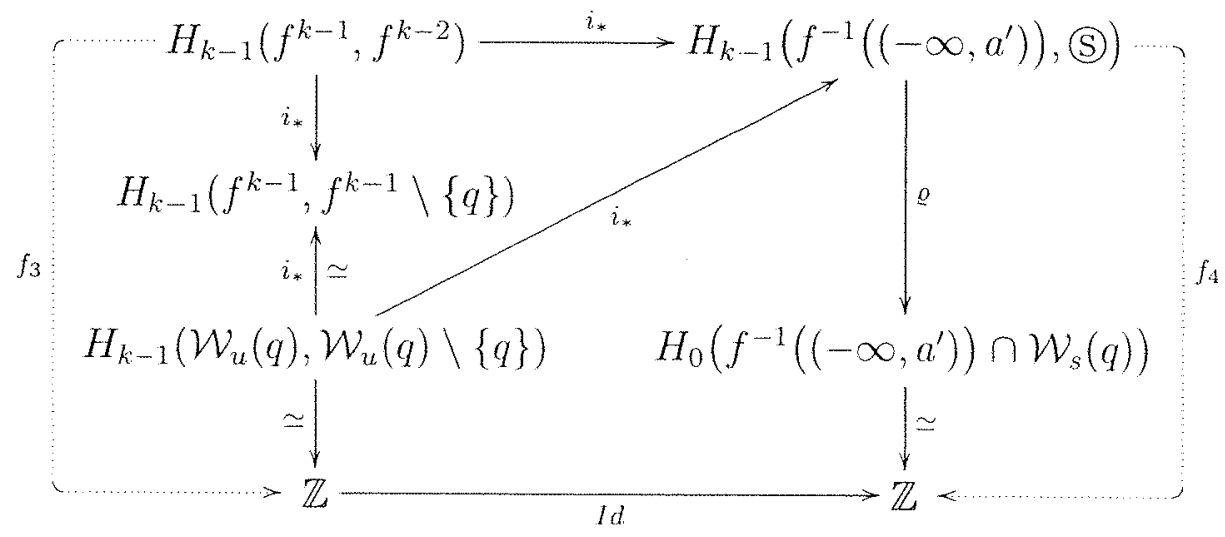

comuta pelo lema (5.1.9) e a funtorialidade da homologia singular. 
Lema 5.1.11. O diagrama:

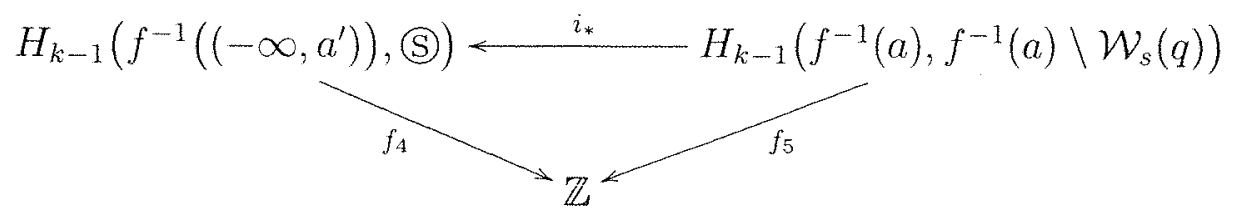

comuta.

Demonstração. Basta observar que o diagrama do lema é equivalente ao seguinte diagrama:

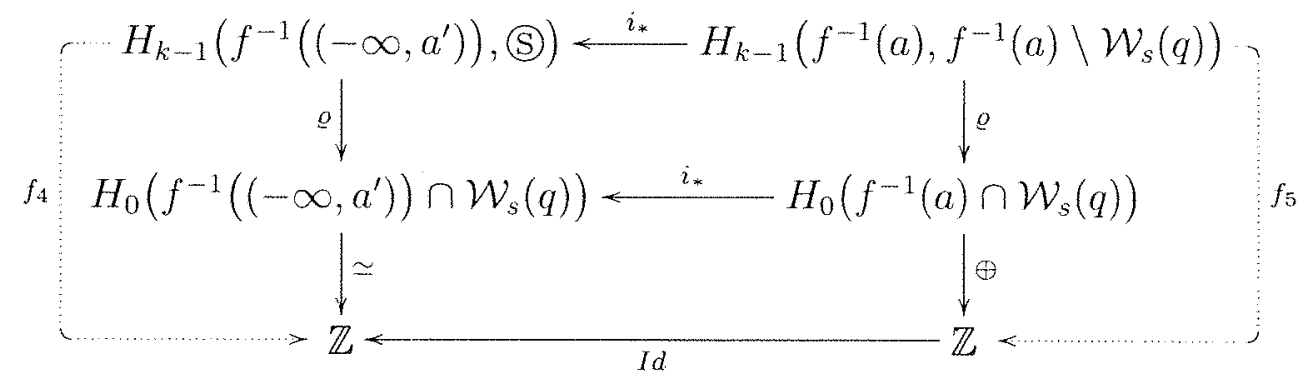

Da observação (5.1.7), temos que o quadrado superior no diagrama acima comuta. A comutatividade do quadrado inferior é imediata.

Finalmente consideremos o seguinte diagrama:

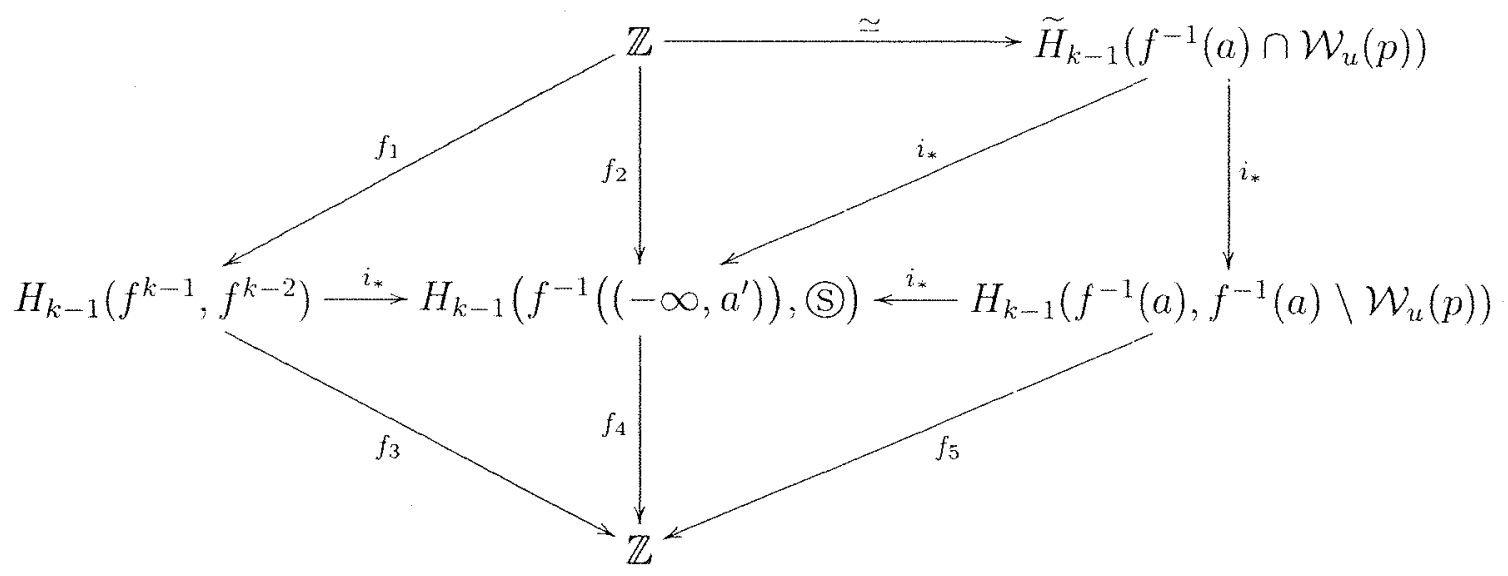

Segue dos lemas $(5.1 .8),(5.1 .10)$ e (5.1.11) que os triângulos do diagrama acima comutam, o quadrado na parte superior direita comuta pela definição do homomorfismo $f_{2}$ e pela funtorialidade da homologia singular. A comutatividade do diagrama acima, garante a comutatividade do diagrama (5.6), e portanto temos provado o resultado desejado. 


\section{Referências Bibliográficas}

[1] Berger M., Gostiaux B., Differential Manifolds, Curves and Surfaces. SpringerVerlag, (1988).

[2] Bredon G., Topology and Geometry. Springer-Verlag,(1993).

[3] Carmo M.P., Geometria Riemanniana. IMPA, (1988).

[4] Dubrovin B.A., Fomenko A.T., Novikov S.P., Modern Geometry - Methods and Applications. Springer-Verlag, (1985).

[5] Floer A., Witten's Complex and Infinite Dimensional Morse Theory. J. Diff. Geom $30(1989), 207-221$.

[6] Mawhin J., Willem M., Critical Point Theory and Hamiltonian Systems. SpringerVerlag, (1989).

[7] Milnor J., Lectures on the h-Cobordism Theorem. Princeton University Press (1965).

[8] Milnor J., Morse Theory. Princeton University Press (1973).

[9] Munkres J. R., Elements of Algebraic Topolgy. The Benjamin/Cummings Publishing Company, Inc., (1984).

[10] Palis Jr. J., Introdução Aos Sistemas Dinâmicos. IMPA (1975).

[11] Piccione P., Tausk D. V., On the Banach differential structure for sets of maps on non-compact domains. Nonlinear Anal. 46 (2001), no. 2, Ser. A: Theory Methods, $245-265$.

[12] Pollack A., Guillemin V., Diferential Topology. Pretince-Hall, (1974).

[13] Schwarz M., Morse Homology. Progress in Math. 111, Birkhäuser, (1993).

[14] Spanier E., Algebraic Topology. McGraw-Hill, New York (1966).

[15] Tausk D.V., Mercuri F., Piccione P., Notes on Morse Theory. $23^{\circ}$ Colóquio Brasileiro De Matemática, IMPA (2001). 